\title{
Infinite-dimensional regularization of McKean-Vlasov equation with a Wasserstein diffusion
}

\author{
Victor MARX *
}

\begin{abstract}
.
Much effort has been spent in recent years on restoring uniqueness of McKeanVlasov SDEs with non-smooth coefficients. As a typical instance, the velocity field is assumed to be bounded and measurable in its space variable and Lipschitzcontinuous with respect to the distance in total variation in its measure variable, see [Jou97, MV, Lac18, CdRF, RZ]. In contrast with those works, we consider in this paper a Fokker-Planck equation driven by an infinite-dimensional noise, inspired by the diffusion models on the Wasserstein space studied in [Kon17b, KvR18, Mar18]. We prove that well-posedness of that equation holds for a drift function that might be only bounded and measurable in its measure argument, provided that a tradeoff is respected between the regularity in the finite-dimensional component and the regularity in the measure argument. In this regard, we show that the higher the regularity of $b$ with respect to its space variable is, the lower regularity we have to assume on $b$ with respect to its measure variable in order to restore uniqueness.
\end{abstract}

Keywords: Wasserstein diffusion, McKean-Vlasov equation, Fokker-Planck equation, regularization properties, restoration of uniqueness, interacting particle system, coalescing particles, Brownian sheet.

AMS MSC 2010: Primary 60H10, 60H15, Secondary 60K35, 60J60, 35Q83

\section{Contents}

0 $\quad$ Introduction $\quad \mathbf{2}$

0.1 Restoration of uniqueness results for McKean-Vlasov equations . . . . . . . . 3

0.2 Diffusions on the Wasserstein space . . . . . . . . . . . . . . . 5

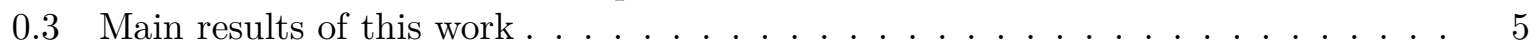

I Regularization of an ill-posed Fokker-Planck equation $\quad 9$

I.1 Construction of the diffusion without drift term . . . . . . . . . . . . . . 10

I.2 Well-posedness of a perturbed Fokker-Planck equation . . . . . . . . . . . . 17

II A continuum of admissible drift functions $\quad 26$

II.1 Description of the class of admissible drift functions $\ldots \ldots \ldots \ldots$

II.2 Definition of the notion of solution . . . . . . . . . . . . . . 27

II.3 Resolution of the SDE when the drift is Lipschitz continuous . . . . . . . . . . . 30

II.4 Resolution of the SDE when the drift is general . . . . . . . . . . . . . 38

\footnotetext{
${ }^{*}$ Université Côte d'Azur, CNRS, Laboratoire J.A. Dieudonné UMR 7351, France.

E-mail: marx@math.uni-leipzig.de
} 


\section{Introduction}

Let us denote by $\mathcal{P}_{2}(\mathbb{R})$ the $L_{2}$-Wasserstein space, consisting in all probability measures $\mu$ on $\mathbb{R}$ such that $\int_{\mathbb{R}} x^{2} \mu(\mathrm{d} x)$ is finite, and by $W_{2}$ the usual Wasserstein distance on $\mathcal{P}_{2}(\mathbb{R})$.

In this paper, we are interested in regularization by noise results for equations in infinite dimension perturbed by infinite-dimensional noises. More precisely, we will consider the following equation:

$$
\left\{\begin{aligned}
\mathrm{d} y_{t}(u) & =b\left(y_{t}(u), \mu_{t}\right) \mathrm{d} t, \quad u \in[0,1], t \in[0, T], \\
\mu_{t} & =\operatorname{Leb}_{[0,1]} \circ y_{t}^{-1},
\end{aligned}\right.
$$

where the unknown $\left(y_{t}\right)_{t \in[0, T]}$ is a time-continuous process such that for each $t \in[0, T], y_{t}$ takes values in the space $L_{2}^{\uparrow}[0,1]$ of non-decreasing square-integrable functions $f:[0,1] \rightarrow \mathbb{R}$. Then the measure-valued process $\left(\mu_{t}\right)_{t \in[0, T]}$ satisfies the following non-linear Fokker-Planck equation on the Wasserstein space $\mathcal{P}_{2}(\mathbb{R})$ :

$$
\partial_{t} \mu_{t}+\operatorname{div}\left(b\left(\cdot, \mu_{t}\right) \mu_{t}\right)=0 .
$$

Remark that (1) and (2) are deterministic equations. If the velocity field $b: \mathbb{R} \times \mathcal{P}_{2}(\mathbb{R}) \rightarrow \mathbb{R}$ is a Lipschitz-continuous function, then equation (1) is well-posed: the proof is based on a fixedpoint method and is similar to the proof of [Szn91, Thm 1.1]. Although existence might hold true in cases where $b$ is less regular, uniqueness often fails to be true when $b$ is not Lipschitzcontinuous, e.g. when for each $\mu \in \mathcal{P}_{2}(\mathbb{R}), b(\mu):=2 \operatorname{sign}(m) \sqrt{|m|}$, where $m:=\int_{\mathbb{R}} x \mu(\mathrm{d} x)$ and $\operatorname{sign}(x):=\mathbb{1}_{\{x \neq 0\}} \frac{x}{|x|}$. That example is derived from the classical Peano counter-example of ill-posedness of a one-dimensional transport equation. Our interest is to restore uniqueness of equation (1) for a certain class of velocity fields $b$ by adding an infinite-dimensional diffusion.

In the first part of this paper, we prove a result of restoration of uniqueness for equation (1) for a perturbative diffusion constructed on $\mathcal{P}_{2}(\mathbb{R})$. That diffusion, which is an infinite-dimensional analogue of a Brownian motion, is constructed as a regularized variant of the Modified Massive Arratia flow introduced by Konarovskyi and von Renesse (see [Kon17b, KvR18, Mar18]). Interestingly enough, diffusions on the Wasserstein space allow to observe averaging effects in infinite dimension: to make it clear, we will assume in this first part that the velocity field $b: \mathbb{R} \times \mathcal{P}_{2}(\mathbb{R}) \rightarrow \mathbb{R}$ is $\mathcal{C}^{2}$-differentiable in the space variable (i.e. the first variable) but only measurable and bounded in the measure variable (i.e. the second variable). This comes in contrast with regularization results in the case where the noise is of the same dimension as the ambiant space, obtained among others by Jourdain [Jou97], Mishura-Veretennikov [MV], Lacker [Lac18], Chaudru de Raynal-Frikha [CdRF] and Röckner-Zhang [RZ]. In those papers, the typical assumptions on the drift function is that $b$ should be bounded and measurable in the space variable and Lipschitz-continuous in total variation distance in the measure variable; in other words, finite-dimensional noises can only average the non-smoothness of a finite-dimensional argument of the drift function.

In the second part of this paper, a connection is made between the result of the first part and the above-mentioned literature. With the aim to interpolate both aforementioned classes of assumptions on $b$, we observe a restoration of uniqueness phenomenon for a continuum of admissible drift functions $b$, as long as a regularity condition is satisfied: roughly speaking, the assumption is $\eta+\frac{3}{2} \delta>\frac{3}{2}$, where $\eta$ is the Sobolev-regularity of $b$ in the space variable and $\delta$ is the Hölder-regularity of $b$ in the measure variable. It should be already noticed at this stage that the results of this second part are obtained at the price of relaxing the related notion of weak solution and of modifying the structure of the noise, by adding an idiosyncratic Brownian motion, as we will explain hereafter.

Before stating the theorems proved in this paper, let us briefly recall important results on restoration of uniqueness for McKean-Valsov equations on the one hand and on construction of diffusions on the Wasserstein space on the other hand. 


\subsection{Restoration of uniqueness results for McKean-Vlasov equations}

As a matter of fact, restoration of uniqueness is now a well-understood phenomenon for classical Itô's SDEs in finite dimension; let us distinguish weak well-posedness results obtained in the aftermath of pioneer work by Stroock and Varadhan (see [SV69, SV79]) and strong well-posedness results, meaning that the solution is adapted to the filtration generated by the noise and that two solutions are almost surely indistinguishable (see Zvonkin [Zvo74], Veretennikov [Ver80], Krylov-Röckner [KR05]). More recently, restoration of uniqueness of PDEs has become an active topic of research. In [FGP10], Flandoli, Gubinelli and Priola have shown that the following transport equation with multiplicative noise

$$
\mathrm{d}_{t} u(t, x)=(b(t, x) \cdot D u(t, x)) \mathrm{d} t+\sum_{i=1}^{d} e_{i} \cdot D u(t, x) \circ d W_{t}^{i} ; \quad u(0, x)=u_{0}(x),
$$

is well-posed for Hölder-continuous drift functions $b$, whereas the transport equation without noise is not necessarily well-posed, see e.g. the counter-example (given in [FGP10]) $b(t, x)=$ $\frac{1}{1-\gamma}\left([x \mid \wedge R)^{\gamma}\right.$ for fixed $R>0$ and $\gamma \in(0,1)$. Many further investigations have been made for SDEs on Hilbert spaces. In a series of papers [DPF10, DPFPR13, DPFPR15], Da Prato, Flandoli, Priola and Röckner proved that pathwise uniqueness holds for an SDE on a Hilbert space $H$ of the form

$$
d X_{t}=\left(A X_{t}+B\left(X_{t}\right)\right) \mathrm{d} t+\mathrm{d} W_{t},
$$

for a certain class of self-adjoint, negative definite operators $A: D(A) \subset H \rightarrow H$, for $W$ a cylindrical Wiener process on $H$ and for $B: H \rightarrow H$ only measurable and locally bounded. For an interesting introduction and a survey of results on regularization by noise phenomena, see also Flandoli's seminal lecture notes [Fla11]. Various other equations in infinite-dimension have also been studied, like e.g. kinetic equations [FFPV17]. Interestingly enough, we quote in this context the recent result of Delarue [Del19] in which some of the above results are used to restore uniqueness to a mean-field game by means of an infinite dimensional common noise of Ornstein-Uhlenbeck type. Although this work shares some motivation with ours, it must be stressed that the dynamics of the particle therein obey an operator $A$ similar to the one that appears in (3). Equivalently, this says that uniqueness is restored but at the price of an extra layer of interactions which is, in contrast to the mean-field one, purely local, arising from the Ornstein-Uhlenbeck noise. The models that we address in the rest of the paper do not have the latter feature.

Obviously, an extensive description of restoration of uniqueness results is out of reach of this introduction, but let us focus in a more detailed fashion on a certain class of equations, namely McKean-Vlasov equations. Let $b: \mathbb{R}^{d} \times \mathcal{P}_{2}\left(\mathbb{R}^{d}\right) \rightarrow \mathbb{R}^{d}$ be a drift function, $\sigma: \mathbb{R}^{d} \times \mathcal{P}_{2}\left(\mathbb{R}^{d}\right) \rightarrow$ $\mathbb{R}^{d \times m}$ be a diffusion matrix and $\left(B_{t}\right)_{t \in[0, T]}$ be a Brownian motion in $\mathbb{R}^{m}$. McKean-Vlasov equation reads as follows

$$
\left\{\begin{aligned}
\mathrm{d} X_{t} & =b\left(X_{t}, \mu_{t}\right) \mathrm{d} t+\sigma\left(X_{t}, \mu_{t}\right) \mathrm{d} B_{t}, \\
\mu_{t} & =\mathcal{L}\left(X_{t}\right)
\end{aligned}\right.
$$

where $\mathcal{L}\left(X_{t}\right)$ denotes the law of $X_{t}$. The coefficients in the stochastic differential equation (4) depend on the distribution of the solution $X_{t}$. That dependence is called mean-field interaction, due to the link with a particle system. Indeed, equation (4) should be regarded as the limit when $N \rightarrow+\infty$ of a system of particles of the following form:

$$
\mathrm{d} X_{t}^{i}=b\left(X_{t}^{i}, \bar{\mu}_{t}^{N}\right) \mathrm{d} t+\sigma\left(X_{t}^{i}, \bar{\mu}_{t}^{N}\right) \mathrm{d} B_{t}^{i}, \quad i=1, \ldots, N,
$$

where $\bar{\mu}_{t}^{N}=\frac{1}{N} \sum_{j=1}^{N} \delta_{X_{t}^{j}}$ and $\left(B_{t}^{i}\right)_{t \in[0, T], 1 \leqslant i \leqslant N}$ are independent Brownian motions, the latter being usually referred to as idiosyncratic noises in order to stress the fact that there are somehow 
proper to a given particle. The trajectory of each particle $\left(X_{t}^{i}\right)_{t \in[0, T]}$ depends on both the current position of the particle and the positions of the other particles, but only via the empirical distribution $\bar{\mu}_{t}^{N}$; that is why this system is called mean-field.

Well-posedness of McKean-Vlasov SDEs has been widely studied. We here provide a tiny example of all the existing references in the field. Generally speaking, existence and uniqueness may be proved by a Picard fixed point argument on the process $\left(\mu_{t}\right)_{t \in[0, T]}$ provided that the coefficients are sufficiently regular, say for instance that they are Lipschitz-continuous in both variables, Lipschitz-continuity with respect to the measure argument being understood with respect to the $L_{2}$-Wasserstein distance. This strategy is made clear in the seminal lecture notes of Sznitman [Szn91]. Variants may be found, see for example (to quote earlier ones) the works of Funaki [Fun84], Gärtner [G̈̈8] or Oelschläger [Oel84]. Interestingly enough, the proof of existence and uniqueness extends to models with a common noise of the form

$$
\mathrm{d} X_{t}=b\left(X_{t}, \mu_{t}\right) \mathrm{d} t+\sigma\left(X_{t}, \mu_{t}\right) \mathrm{d} B_{t}+\sigma_{0}\left(t, X_{t}, \mu_{t}\right) \mathrm{d} W_{t},
$$

with the constraint that $\mu_{t}$ now matches the conditional law of $X_{t}$ given the realization of $W$, where $\left(W_{t}\right)_{t \in[0, T]}$ is a new Brownian motion, independent of $\left(B_{t}\right)_{t \in[0, T]}$ and of dimension $m_{0}$, and $\sigma_{0}$ stands for a new volatility coefficient defined in the same manner as $\sigma$. Importantly, $\mu_{t}$ becomes random under the presence of $W$. The terminology common noise is better understood when we write down the analogue of (5), which reads:

$$
\mathrm{d} X_{t}^{i}=b\left(X_{t}^{i}, \bar{\mu}_{t}^{N}\right) \mathrm{d} t+\sigma\left(X_{t}^{i}, \bar{\mu}_{t}^{N}\right) \mathrm{d} B_{t}^{i}+\sigma_{0}\left(X_{t}^{i}, \bar{\mu}_{t}^{N}\right) \mathrm{d} W_{t}, \quad i=1, \ldots, N .
$$

The key fact here is that all the particles are driven by the same noise $\left(W_{t}\right)_{t \in[0, T]}$, which is of course assumed to be independent of the collection $\left(B_{t}^{i}\right)_{t \in[0, T], 1 \leqslant i \leqslant N}$. The reader may have a look at the works of Vaillancourt [Vai88], Dawson and Vaillancourt [DV95], Kurtz and Xiong [KX99, KX04] or Coghi and Flandoli [CF16] for more details on (6) and (7).

Let us describe restoration of uniqueness phenomena for McKean-Vlasov equations. Wellposedness may fail to be true for the "deterministic" equation (i.e. $\sigma \equiv 0$ in (4) in which case the randomness only comes from the initial condition) when the drift term $b=b(x, \mu)$ is not regular enough. Existing results in the field show that it is possible to require $b$ to be merely measurable and bounded in the space variable $x$ and Lipschitz-continuous in the measure variable $\mu$, with respect to the topology generated by the total variation distance $\mathrm{d}_{\mathrm{TV}}$, defined by

$$
\mathrm{d}_{\mathrm{TV}}(\mu, \nu):=2 \sup \left\{\left|\int_{\mathbb{R}^{d}} f \mathrm{~d} \mu-\int_{\mathbb{R}^{d}} f \mathrm{~d} \nu\right| ; f: \mathbb{R}^{d} \rightarrow \mathbb{R} \text { measurable, }\|f\|_{L_{\infty}} \leqslant 1\right\},
$$

which is finer than the topology generated by the Wasserstein distance $W_{2}$. In particular, Jourdain [Jou97] has proved that restoration of uniqueness holds in a weak sense for McKean-Vlasov equation (4) in a case where $\sigma \equiv 1$ and $b$ is bounded, measurable and Lipschitz-continuous in its measure variable with respect to $\mathrm{d}_{\mathrm{TV}}$. Recently, several papers have improved the results, proving well-posedness for more general coefficients $\sigma$ in cases where $\sigma$ does not depend on $\mu$ [MV, Lac18, CdRF, RZ]. In [MV], Mishura and Veretennikov have in particular shown pathwise uniqueness under Lipschitz-continuity assumptions on $b$ with respect to the measure variable and on $\sigma$ with respect to the space variable. In [Lac18], Lacker gives a short proof of well-posedness relying on a fixed-point argument. Röckner and Zhang [RZ] have extended the results to the case of unbounded coefficients with suitable integrability properties, in the sense of Krylov-Röckner [KR05]. Let us emphasize once more that $b$ is assumed to be at least Lipschitz-continuous with respect to the measure-variable in total variation distance. This assumption might presumably be explained by the fact that the finite dimensional noise $B$ cannot have a regularizing effect on the infinitely many directions of the measure argument of $b$; that is one of the reasons that drives us to study more precisely the effect of a noise defined on the Wasserstein space $\mathcal{P}_{2}(\mathbb{R})$. 


\subsection{Diffusions on the Wasserstein space}

Before defining the diffusion model that we use in this text, let us briefly introduce the preexisting models that have inspired our construction. In [vRS09], von Renesse and Sturm constructed a so-called Wasserstein diffusion on the space of probability measures on $[0,1]$, that is a Markovian stochastic process $\left(\mu_{t}\right)_{t \in[0, T]}$ with a reversibility property with respect to an entropic measure on $\mathcal{P}_{2}([0,1])$. Interestingly, the dynamics of $\left(\mu_{t}\right)_{t \in[0, T]}$ are similar to the dynamics of a standard Brownian motion, in the sense that the large deviations in small time are given by the Wasserstein distance $W_{2}$ and the martingale term that arises when expanding any smooth function $\varphi$ of the measure argument along the process has exactly the square norm of the Wasserstein gradient of $\varphi$ as local quadratic variation. Stochastic processes owning those diffusive features are various and several were studied in recent years. We decide in this paper to construct a diffusion inspired by the nice model of coalescing particles called Modified Massive Arratia flow: in [Kon11, Kon17b], Konarovskyi introduces a diffusion model on $\mathcal{P}_{2}(\mathbb{R})$ consisting in a modification of Arratia's system of coalescing particles on the real line. To wit, in Konarovskyi's model, each particle carries a mass determining its quadratic variation and moves independently of the other particles as long as it does not collide with another. To make it clear, at each collision between two particles, both particles stick together and form a unique new particle with a mass equal to the sum of the masses of both incident particles. At each time, the quadratic variation increment of a particle is given by the inverse of its mass. That model satisfies interesting properties, studied by Konarovskyi and von Renesse in [Kon17b, Kon17a, KvR18], including an Itô-like formula and a Varadhan-like formula, with the Wasserstein distance $W_{2}$ playing the analogous role of the Euclidean metric for the standard Brownian motion. Moreover, those dynamics have a canonical representation as a process of quantile functions (or increasing rearrangement functions) $\left(y_{t}\right)_{t \in[0, T]}: \forall u \in[0,1], y_{t}(u):=\sup \left\{x \in \mathbb{R}: \mu_{t}((-\infty, x]) \leqslant u\right\}$.

Despite a simple construction and the diffusive properties described above, the question of the uniqueness of Konarovskyi's model (not only pathwise uniqueness but also uniqueness in law) remains - as far as the author knows - open. In particular, it has a singularity at time $t=0^{+}$: if $\mu_{0}$ has a density with respect to the Lebesgue measure, then almost surely for every $t>0$, the probability measure $\mu_{t}$ is a finite weighted sum of Dirac masses or, in other words, the quantile function $y_{t}$ is a step function. In [Mar18], the author overcomes lack of uniqueness by modifying Konarovskyi's model, replacing the coalescing procedure by a system of particles interacting at short range; among others, whenever the initial condition has a regular density, the solution itself remains an absolutely continuous measure. The author has proved in [Mar18] the convergence of that mollified model to the Modified Massive Arratia flow. The diffusion used in this work to regularize Fokker-Planck equation is directly inspired from the works [Kon17b, Mar18].

\subsection{Main results of this work}

This paper is divided into two parts and gives two complementary results of well-posedness, in a weak sense, of perturbed Fokker-Planck equations. First, we will address the case of a drift function $b$ with low regularity in its measure variable but $C^{2}$-regularity in space. Second, we will treat a continuum of admissible velocity fields $b$ that somehow interpolates the assumptions of the first part and those of [Jou97, MV, Lac18, CdRF, RZ]. The structure of the equations are almost similar in both parts, up to the addition of an idiosyncratic noise in the second part, which comes up with a slightly more general notion of weak solution.

\subsubsection{Restoration of uniqueness for a velocity field merely measurable in its mea- sure argument}

Let $T \in(0,+\infty)$ be a fixed time. 
Let us consider the Fokker-Planck equation (1) perturbed by a diffusive noise: for each $t \in[0, T]$ and each $u \in[0,1]$,

$$
\left\{\begin{aligned}
\mathrm{d} y_{t}(u) & =b\left(y_{t}(u), \mu_{t}\right) \mathrm{d} t+\frac{1}{\left(\int_{0}^{1} \varphi\left(y_{t}(u)-y_{t}(v)\right) \mathrm{d} v\right)^{1 / 2}} \int_{\mathbb{R}} f(k) \Re\left(e^{-i k y_{t}(u)} \mathrm{d} w(k, t)\right) ; \\
\mu_{t} & =\operatorname{Leb}_{[0,1]} \circ y_{t}^{-1},
\end{aligned}\right.
$$

with initial condition $y_{0}=g$. That equation describes a system of particles in interaction; for every $u \in[0,1],\left(y_{t}(u)\right)_{t \in[0, T]}$ denotes the trajectory of the particle indexed by $u$ and for every $t \in[0, T], \mu_{t}$ is the distribution of the cloud of particles. There is a mean-field interaction in SDE (8), both through a drift term $b$ which takes as an argument the probability measure $\mu_{t}$, and through the diffusion term, since the denominator $\left(\int_{0}^{1} \varphi\left(y_{t}(u)-y_{t}(v)\right) \mathrm{d} v\right)^{1 / 2}$ also depends on the distribution on the real line of the cloud of particles.

Let us briefly describe the different terms appearing in equation (8).

- as in [Kon17b, Mar18], the unknown $\left(y_{t}\right)_{t \in[0, T]}$ is a time-continuous stochastic process such that for each $t \in[0, T], y_{t}$ is a random variable with values in the space $L_{2}^{\uparrow}[0,1]$. Recall that for each $t \in[0, T], u \in[0,1] \mapsto y_{t}(u)$ can be seen as the quantile function associated to the measure $\mu_{t}=\operatorname{Leb}_{[0,1]} \circ y_{t}^{-1}$ belonging to $\mathcal{P}_{2}(\mathbb{R})$. Importantly, it means that we are studying stochastic processes $\left(\mu_{t}\right)_{t \in[0, T]}$ in $\mathcal{P}_{2}(\mathbb{R})$ that admit a canonical representation in the form of a tractable process of quantile functions.

- the function $b: \mathbb{R} \times L_{2}[0,1] \rightarrow \mathbb{R}$ will be called the drift function or velocity field.

- the second term appearing in (8) is the diffusion term. This term looks like the process introduced by the author in [Mar18]. We refer to Remark 1 below to explain the reasons why we slightly modify the shape of the diffusion here. It consists of several parts:

$\triangleright$ the complex-valued Brownian sheet $w$ is defined by $w:=w^{\Re}+i w^{\Im}$, where $w^{\Re}$ and $w^{\Im}$ are two independent real Brownian sheets on $\mathbb{R} \times[0, T]$ and $i=\sqrt{-1}$. To make it clear, $\Re\left(e^{-i k y_{t}(u)} \mathrm{d} w(k, t)\right)=\cos \left(k y_{t}(u)\right) \mathrm{d} w^{\Re}(k, t)+\sin \left(k y_{t}(u)\right) \mathrm{d} w^{\Im}(k, t)$. The definition of Brownian sheets will be recalled at the beginning of Part I of this paper.

$\triangleright$ the function $f$ will typically be of the form $f_{\alpha}(k)=\frac{1}{\left(1+k^{2}\right)^{\alpha / 2}}$. The higher $\alpha$ is, the smoother the diffusion term is with respect to the space variable $u$.

$\triangleright$ we denote by $m_{t}(u):=\int_{0}^{1} \varphi\left(y_{t}(u)-y_{t}(v)\right) \mathrm{d} v$ the mass function. In order to avoid problems of cancellation of the mass, we will only consider in this text functions $\varphi$ which are positive everywhere on $\mathbb{R}$. A typical example will be the Gaussian density. Our results will also include the case where the mass is constant, i.e. when $\varphi \equiv 1$.

In words, the role of $\varphi$ is to tune the local variance of the particle. This is similar to the models presented by Konarovskyi [Kon17b] and by the author [Mar18], where the quadratic variation of $\left(y_{t}(u)\right)_{t \in[0, T]}$ is proportional to $\int_{0}^{t} \frac{\mathrm{d} s}{m_{s}(u)}$. In order to make the comparison more precise, we may compute the local covariation field of the martingale component in (8), namely, for any two $u, u^{\prime} \in[0,1]$,

$$
\begin{aligned}
\mathrm{d}\left\langle y \cdot(u), y \cdot\left(u^{\prime}\right)\right\rangle_{t} & =\frac{1}{m_{t}(u)^{1 / 2} m_{t}\left(u^{\prime}\right)^{1 / 2}} \int_{\mathbb{R}} f^{2}(k) \cos \left(k\left(y_{t}(u)-y_{t}\left(u^{\prime}\right)\right)\right) \mathrm{d} k \mathrm{~d} t \\
& =\frac{\Re\left(\mathcal{F}\left(f^{2}\right)\right)\left(y_{t}(u)-y_{t}\left(u^{\prime}\right)\right)}{m_{t}(u)^{1 / 2} m_{t}\left(u^{\prime}\right)^{1 / 2}} \mathrm{~d} t,
\end{aligned}
$$

where $\mathcal{F}\left(f^{2}\right)$ stands for the Fourier transform of $f^{2}$. Interestingly enough, formula (9) may be compared with the covariation of the Modified Massive Arratia flow (see [Kon17b]):

$$
\left\langle y \cdot(u), y \cdot\left(u^{\prime}\right)\right\rangle_{t}=\int_{\tau_{u, u^{\prime}}}^{t \wedge \tau_{u, u^{\prime}}} \frac{1}{m_{s}(u)} \mathrm{d} s,
$$


where $\tau_{u, u^{\prime}}:=\inf \left\{t \geqslant 0: y_{t}(u)=y_{t}\left(u^{\prime}\right)\right\} \wedge T$ stands for the collision stopping time and $m_{s}(u):=$ $\int_{0}^{1} \mathbb{1}_{\left\{\tau_{u, v} \leqslant s\right\}} \mathrm{d} v$ stands for the Lebesgue measure of the particles which have already coalesced with particle $u$ at time $s$. For instance, whenever (say to make it simple) $f(k)=f_{1}(k)=\frac{1}{\left(1+k^{2}\right)^{1 / 2}}$, $\mathcal{F}\left(f^{2}\right)(x)$ behaves like $\exp (-|x|)$, which shows that the range of interaction in (8) is infinite but decays exponentially fast. By computing the Fourier transform with a residue formula, the latter may be shown to remain true whenever $f(k)=f_{n}(k)$ for any integer $n \geqslant 1$, which proves that this new model shares some of the features of the approximation introduced in [Mar18] but has a longer interaction range.

The first main result of this paper is the following theorem, stating well-posedness of equation (8). The assumptions on the velocity field $b$ are simplified here, we refer to Definition 12 and to Theorem 22 for more details. We denote by $\partial_{1}^{(j)} b, j=1,2$, the first two derivatives of $x \mapsto b(x, \mu)$ at fixed $\mu$.

Theorem 1. Let $g$ be a strictly increasing $\mathcal{C}^{1}$-function. Let $f: \mathbb{R} \rightarrow \mathbb{R}$ be defined by $f(k)=$ $f_{\alpha}(k):=\frac{1}{\left(1+k^{2}\right)^{\alpha / 2}}$, with $\alpha>\frac{3}{2}$. Let $b: \mathbb{R} \times \mathcal{P}_{2}(\mathbb{R}) \rightarrow \mathbb{R}$ be a bounded measurable function such that for each $\mu \in \mathcal{P}_{2}(\mathbb{R}), x \mapsto b(x, \mu)$ is twice continuously differentiable and $\partial_{1} b$ and $\partial_{1}^{(2)} b$ are uniformly bounded on $\mathbb{R} \times \mathcal{P}_{2}(\mathbb{R})$. Then there is a unique weak solution to equation (8).

The proof of Theorem 1 relies on Girsanov's Theorem. The main issue is to write the drift term $b$ as a perturbation of the noise. To achieve this goal, we have to invert the diffusion coefficient; more precisely, we will resolve the following equation: find a complex-valued process $\left(h_{t}\right)_{t \in[0, T]}$ satisfying for every $x \in \mathbb{R}$ and $t \in[0, T]$

$$
b\left(x, \mu_{t}\right)=\frac{1}{\left(\int_{0}^{1} \varphi\left(x-y_{t}(v)\right) \mathrm{d} v\right)^{1 / 2}} \int_{\mathbb{R}} f_{\alpha}(k) \Re\left(e^{-i k x} h_{t}(k)\right) \mathrm{d} k .
$$

Thanks to the fact (and this is our rationale for it) that we chose an interaction kernel in the diffusion term of (8) in a Fourier-like shape, $h$ can be defined as an inverse Fourier transform:

$$
h_{t}(k)=\frac{1}{f_{\alpha}(k)} \mathcal{F}^{-1}\left(b\left(\cdot, \mu_{t}\right) \cdot\left(\varphi * p_{t}\right)^{-1 / 2}\right)(k),
$$

where $p_{t}$ denotes the density of the measure $\mu_{t}$. To apply Girsanov's Theorem, $h$ should belong to $L_{2}(\mathbb{R} ; \mathbb{C})$; that is why we assume in Theorem 1 some regularity of $b$ with respect to the variable $x$. Remark that the higher $\alpha$ is, the more difficult it is to invert the kernel. It highlights a balance between the regularity of the process $y$ and the integrability of the Fourier inverse of $h$.

Remark 1. Let us explain what happens when we perturb Fokker-Planck equation (1) with the diffusion of [Mar18]:

$$
\mathrm{d} y_{t}(u)=b\left(y_{t}(u), \mu_{t}\right) \mathrm{d} t+\frac{1}{\int_{0}^{1} \varphi^{2}\left(y_{t}(u)-y_{t}(v)\right) \mathrm{d} v} \int_{0}^{1} \varphi\left(y_{t}(u)-y_{t}\left(u^{\prime}\right)\right) \mathrm{d} w\left(u^{\prime}, t\right) .
$$

Then, the inversion problem consists in finding an $L_{2}[0,1]$-process $\left(h_{t}\right)_{t \in[0, T]}$ satisfying for every $x \in \mathbb{R}$ and $t \in[0, T]$

$$
b\left(x, \mu_{t}\right)=\frac{1}{\int_{0}^{1} \varphi^{2}\left(x-y_{t}(v)\right) \mathrm{d} v} \int_{0}^{1} \varphi\left(x-y_{t}\left(u^{\prime}\right)\right) h_{t}\left(u^{\prime}\right) \mathrm{d} u^{\prime},
$$

and equivalently in solving for every $x \in \mathbb{R}$ and $t \in[0, T]$

$$
\left(h_{t} \circ F_{t}\right)(x)=\frac{1}{p_{t}(x)} \mathcal{F}^{-1}\left(\frac{\mathcal{F}\left(b\left(\cdot, \mu_{t}\right) \cdot\left(\varphi^{2} * p_{t}\right)\right)}{\mathcal{F}(\varphi)}\right)(x),
$$


where $F_{t}$ (resp. $p_{t}$ ) stands for the c.d.f. (resp. the density) associated to $\mu_{t}$. There are two major hindrances with equation (11). The first one is the division by the density $p_{t}$ : $p_{t}$ is equal to zero outside the support of $\mu_{t}$ and $\frac{\mathcal{F}\left(b\left(\cdot, \mu_{t}\right) \cdot\left(\left(\varphi^{2}\right) * p_{t}\right)\right)}{\mathcal{F}(\varphi)}$ has no chance to be smooth enough so that its inverse Fourier transform has compact support. It led us to change the model so that the integral in the right-hand side of (10) is written as a Fourier transform. The second problem with $(11)$ is the division by $\mathcal{F}(\varphi)$. In the case where $\varphi$ is a Gaussian density, $\frac{1}{\mathcal{F}(\varphi)(k)}$ behaves like $e^{k^{2} / 2}$. Even if $b$ is $\mathcal{C}^{\infty}$ with respect to its first variable, this would not be sufficient to obtain $L_{2}$-integrability of $h$. Let us try to reduce the regularity of $\varphi$ : if $\varphi(x)=e^{-|x|}$, then $\frac{1}{\mathcal{F}(\varphi)(k)}=1+k^{2}$. Nevertheless, the density $p_{t}$ cannot be of class $\mathcal{C}^{1}$ with this choice of function $\varphi$ (we refer to Remark 11). Thus even with smooth functions $b$, the regularity of $\varphi^{2} * p_{t}$ is not sufficient to compensate for the term $\frac{1}{\mathcal{F}(\varphi)}$ and to insure that $h_{t}$ belongs to $L_{2}$. In order to solve this problem, we chose to consider two different functions $f_{\alpha}$ and $\varphi$ respectively at numerator and denominator of the diffusive part of (8); this trick allows us to choose different regularities on $f_{\alpha}$ and on $\varphi$.

\subsubsection{Restoration of uniqueness under a regularity assumption in both arguments of the velocity field}

The second main result of this text is a well-posedness result for a continuum of admissible drift functions $b$ that interpolates the assumptions of Theorem 1 and the assumptions usually made for McKean-Vlasov equations with finite-dimensional noise, namely $b$ Lipschitz-continuous with respect to $\mathrm{d}_{\mathrm{TV}}$ in its measure variable and $b$ bounded and measurable in its space argument (see [Jou97, MV, CdRF, Lac18, RZ]). Importantly, we succeed to do so at the price of relaxing in a dramatic manner the structure of the noise in hand and of the related notion of solution, adding in particular a new idiosyncratic noise denoted by $\beta$. In particular, as we explain below, the diffusion used to obtain the latter interpolation result does not fit the main features of the models in [Kon17b, Mar18] and in the first part. Among others, we lose here the underlying property of monotonicity, meaning that the solution can no longer be seen as the quantile function of the associated measure-valued process. Nevertheless, we feel that this interpolation argument is important to make the connection between the regularity assumptions of Theorem 1 and those used in the pre-existing literature.

Here is our new model. Let $\mu_{0} \in \mathcal{P}_{2}(\mathbb{R})$ be an initial condition and $\xi$ be a random variable with law $\mu_{0}$. Let $w:=w^{\Re}+i w^{\Im}$ be a complex-valued Brownian sheet defined as in equation (8). Let $\left(\beta_{t}\right)_{t \in[0, T]}$ be a Brownian motion independent of $(w, \xi)$. Let us consider the following McKean-Vlasov SDE with constant mass:

$$
\left\{\begin{aligned}
\mathrm{d} z_{t} & =b\left(z_{t}, \mu_{t}\right) \mathrm{d} t+\int_{\mathbb{R}} f(k) \Re\left(e^{-i k z_{t}} d w(k, t)\right)+\mathrm{d} \beta_{t}, \\
\mu_{t} & =\mathcal{L}^{\mathbb{P}}\left(z_{t} \mid \mathcal{G}_{t}^{\mu, W}\right), \quad(\mu, w) \Perp(\beta, \xi) \\
z_{0} & =\xi, \quad \mathcal{L}^{\mathbb{P}}(\xi)=\mu_{0},
\end{aligned}\right.
$$

where $\left(\mathcal{G}_{t}^{\mu, W}\right)_{t \in[0, T]}$ is the filtration generated by the Brownian sheet $w$ and by the measurevalued process $\left(\mu_{t}\right)_{t \in[0, T]}$ itself. Whereas $w$ is seen as a common noise, the Brownian motion $\beta$ is seen here as an idiosyncratic source of randomness and $\mu_{t}$ can be seen as the law of $z_{t}$ with respect to the randomness carrying both the initial condition and the idiosyncratic noise. The addition of the new source of randomness $\beta$ is easily understood: similar to the Brownian motion in standard SDEs, it allows to mollify the drift in the space variable $x$. As for the conditioning in the identity $\mu_{t}=\mathcal{L}^{\mathbb{P}}\left(z_{t} \mid \mathcal{G}_{t}^{\mu, W}\right)$, it must be compared with our presentation of McKean-Vlasov equations with a common noise, see (6). The main difference between both is that the conditioning now involves $\mu$ itself: this comes from the fact we will allow for weak solutions, namely for solutions for which $\mu$ may not be adapted with respect to the common noise $w$. In fact, the latter causes some 
technical difficulties in the proofs. In particular, it requires to work with solutions that satisfy an additional assumption: the observation of $z$ cannot bias the future realizations of $\mu, w$ and $\beta$. That new requirement is known as the compatibility condition and has been often used in the study of weak solutions to stochastic equations (see [Kur07, Kur14]). We refer to Section II.2 for a complete definition of the notion of weak compatible solution.

Here is our result. Let $\eta>0$ and $\delta \in[0,1]$. Let us consider the drift function $b: \mathbb{R} \times \mathcal{P}_{2}(\mathbb{R}) \rightarrow$ $\mathbb{R}$ in the class $\left(H^{\eta}, \mathcal{C}^{\delta}\right)$. The definition of that class of admissible drift functions is given in Section II.1, but roughly speaking, it contains functions $b$ such that for every fixed $\mu, x \mapsto b(x, \mu)$ belongs to the Sobolev space $H^{\eta}(\mathbb{R})$ with a Sobolev norm uniform in $\mu$, and for every fixed $x$, $\mu \mapsto b(x, \mu)$ is $\delta$-Hölder continuous in $\mathrm{d}_{\mathrm{TV}}$. the Hölder norm being uniform in $x$. Then, we have the following statement:

Theorem 2. Let $\eta>0$ and $\delta \in[0,1]$ be such that $\eta>\frac{3}{2}(1-\delta)$ and let $b$ be of class $\left(H^{\eta}, \mathcal{C}^{\delta}\right)$. Let $f: \mathbb{R} \rightarrow \mathbb{R}$ be defined by $f(k)=f_{\alpha}(k):=\frac{1}{\left(1+k^{2}\right)^{\alpha / 2}}$, with $\frac{3}{2}<\alpha \leqslant \frac{\eta}{1-\delta}$. Then existence and uniqueness of a weak compatible solution to equation (12) hold.

The condition $\eta>\frac{3}{2}(1-\delta)$ quantifies the minimal regularity that is needed, with our approach, to restore uniqueness. If $b$ is Lipschitz-continuous in total variation distance with respect to $\mu(\delta=1)$, then almost no regularity of $b$ in $x$ is needed $(\eta>0)$ : it is close to the assumptions of [Jou97, MV, CdRF, Lac18, RZ]. If $b$ is only uniformly bounded in $\mu(\delta=0)$, then $x \mapsto b(x, \mu)$ should belong to $H^{\eta}(\mathbb{R})$ for some $\eta>\frac{3}{2}$, which is slightly stronger than the assumption made in Theorem 1. In particular, it holds if $\eta=\delta=\frac{2}{3}$, in a case where $b$ is not Lipschitz-continuous in any variable.

Organisation of the paper. The part I of this work will be devoted to the construction of the above-mentioned variant of a diffusive model on the Wasserstein space and to the proof of the regularization result stated in Theorem 1. In the part II, we describe the trade-off between regularity in the space and in the measure variable and we prove Theorem 2.

Notations. Throughout this paper, we will always denote by $C_{M}$ the constants depending on $M$, even if they change from one line to the next. We will also denote by $\langle k\rangle:=\left(1+k^{2}\right)^{1 / 2}$.

\section{Regularization of an ill-posed Fokker-Planck equation}

Let $\left(\Omega, \mathcal{G},\left(\mathcal{G}_{t}\right)_{t \in[0, T]}, \mathbb{P}\right)$ be a filtered probability space. Assume that $\mathcal{G}_{0}$ contains all the $\mathbb{P}$-null sets.

Let us recall the definition, given by Walsh [Wal86, p.269], of a real-valued Brownian sheet on $\mathbb{R} \times[0, T]$. We call white noise on $\mathbb{R} \times[0, T]$ any random set function $W$ defined on the set of Borel subsets of $\mathbb{R} \times[0, T]$ with finite Lebesgue measure such that

- for any $A \in \mathcal{B}(\mathbb{R} \times[0, T])$ with finite Lebesgue measure, $W(A)$ is a normally distributed random variable with zero mean and with variance equal to $\operatorname{Leb}(A)$;

- for any disjoint subsets $A$ and $B \in \mathcal{B}(\mathbb{R} \times[0, T])$ with finite Lebesgue measures, $W(A)$ and $W(B)$ are independent and $W(A \cup B)=W(A)+W(B)$.

The random function $w: \mathbb{R} \times[0, T] \rightarrow R$, defined for every $t \in[0, T]$ by $w(k, t)=W([0, k] \times[0, t])$ if $k \geqslant 0$ and $w(k, t)=W([k, 0] \times[0, t])$ if $k<0$, is called Brownian sheet on $\mathbb{R} \times[0, T]$. Let us fix two independent $\left(\mathcal{G}_{t}\right)_{t \in[0, T]}$-adapted Brownian sheets $w^{\Re}$ and $w^{\Im}$ on $\mathbb{R} \times[0, T]$. The process $w=w^{\Re}+i w^{\Im}$ is called complex-valued Brownian sheet on $\mathbb{R} \times[0, T]$. We refer to [Mar18, Theorem 1.1] for an explanation as to how Brownian sheets are naturally related to Konarovskyi's model. 
Let us rewrite hereafter equation (8): we are looking for a solution $\left(y_{t}\right)_{t \in[0, T]}$ with values in $L_{2}^{\uparrow}[0,1]$ such that for any $u \in[0,1]$ and any $t \in[0, T]$,

$$
\left\{\begin{aligned}
d y_{t}(u) & =b\left(y_{t}(u), \mu_{t}\right) \mathrm{d} t+\frac{1}{m_{t}(u)^{1 / 2}} \int_{\mathbb{R}} f(k)\left(\cos \left(k y_{t}(u)\right) \mathrm{d} w^{\Re}(k, t)+\sin \left(k y_{t}(u)\right) \mathrm{d} w^{\Im}(k, t)\right), \\
\mu_{t} & =\operatorname{Leb}_{[0,1]} \circ y_{t}^{-1}, \\
y_{0} & =g
\end{aligned}\right.
$$

with $m_{t}(u)=\int_{0}^{1} \varphi\left(y_{t}(u)-y_{t}(v)\right) \mathrm{d} v$. Let us define and comment the different terms appearing in that equation. First, the space $L_{2}^{\uparrow}[0,1]$ is the set of square-integrable non-decreasing functions from $[0,1]$ to $\mathbb{R}$. To wit, we are looking for solutions to (13) such that for each time $t \in[0, T]$, the map $u \mapsto y_{t}(u)$ is non-decreasing; therefore, it is the quantile function associated to the measure $\mu_{t}$. In other words, equation (13) is describing the random dynamics of the process $\left(\mu_{t}\right)_{t \in[0, T]}$ via its canonical representation in terms of a quantile function process $\left(y_{t}\right)_{t \in[0, T]}$. We will assume that the initial condition belongs to $L_{2+}^{\uparrow}[0,1]$, the set of non-decreasing functions $g:[0,1] \rightarrow \mathbb{R}$ such that there is $p>2$ satisfying $\int_{0}^{1}|g(u)|^{p} \mathrm{~d} u<+\infty$. The map $\varphi: \mathbb{R} \rightarrow \mathbb{R}$ is an even function of class $\mathcal{C}^{\infty}$, decreasing on $[0,+\infty)$ and such that for every $x \in[0,+\infty)$, $\varphi(x)>0$. Typical examples of functions $\varphi$ are the constant function $\varphi \equiv 1$ and the Gaussian density $\varphi(x)=\frac{1}{\sqrt{2 \pi}} e^{-x^{2} / 2}$. The map $f: \mathbb{R} \rightarrow \mathbb{R}$ is an even and square integrable function. The precise assumptions on the drift function $b: \mathbb{R} \times \mathcal{P}_{2}(\mathbb{R}) \rightarrow \mathbb{R}$ will be given later.

It should be once more emphasized that, due to the presence of the noise $w$, the process $\left(\mu_{t}\right)_{t \in[0, T]}$ is random. More precisely, by a straightforward computation of Itô's formula, it can be shown that the process $\left(\mu_{t}\right)_{t \in[0, T]}$ satisfies the following SPDE:

$$
\begin{aligned}
\mathrm{d} \mu_{t}+\partial_{x}\left(b\left(\cdot, \mu_{t}\right) \mu_{t}\right) \mathrm{d} t= & \frac{1}{2}\|f\|_{L_{2}(\mathbb{R})}^{2} \partial_{x x}^{2}\left(\frac{\mu_{t}}{\varphi * \mu_{t}}\right) \mathrm{d} t \\
& -\partial_{x}\left(\frac{\mu_{t}}{\left(\varphi * \mu_{t}\right)^{1 / 2}} \int_{\mathbb{R}} f(k) \Re\left(e^{-i k \cdot} \mathrm{d} w(k, t)\right)\right) .
\end{aligned}
$$

We recognize on the left-hand side of equation (14) a Fokker-Planck equation, with a diffusive perturbation appearing on the right-hand side due to the addition of a noise. Here, $\varphi * \mu_{t}:=$ $\int_{\mathbb{R}} \varphi(\cdot-x) \mathrm{d} \mu_{t}(x)$ represents the mass function. If $\varphi$ is close to the indicator function $\mathbb{1}_{0}$ and $b \equiv 0$, then equation (14) becomes very similar to the SPDE obtained by Konarovskyi and von Renesse for their model [KvR18]:

$$
\mathrm{d} \mu_{t}=\Gamma\left(\mu_{t}\right) \mathrm{d} t+\operatorname{div}\left(\sqrt{\mu_{t}} d W_{t}\right),
$$

where $\Gamma$ is defined as $\langle f, \Gamma(\nu)\rangle:=\frac{1}{2} \sum_{x \in \operatorname{Supp}(\nu)} f^{\prime \prime}(x)$.

Let us first, in Section I.1, construct the diffusion, i.e. solve equation (13) when $b \equiv 0$. Then, in Section I.2, we will prove well-posedness of equation (13) under the assumptions given in Theorem 1.

\section{I.1 Construction of the diffusion without drift term}

The aim of this section is to study the solvability of the equation without drift, i.e. equation (13) when $b \equiv 0$ :

$$
\begin{aligned}
y_{t}(u)=g(u) & +\int_{0}^{t} \frac{1}{m_{s}(u)^{1 / 2}} \int_{\mathbb{R}} \cos \left(k y_{s}(u)\right) f(k) \mathrm{d} w^{\Re}(k, s) \\
& +\int_{0}^{t} \frac{1}{m_{s}(u)^{1 / 2}} \int_{\mathbb{R}} \sin \left(k y_{s}(u)\right) f(k) \mathrm{d} w^{\Im}(k, s),
\end{aligned}
$$


with $m_{s}(u)=\int_{0}^{1} \varphi\left(y_{s}(u)-y_{s}(v)\right) \mathrm{d} v$.

In Paragraph I.1.1, we will introduce an auxiliary equation where the function $\varphi$ is replaced by a truncated function $\varphi_{M}$ so that the diffusion coefficient is bounded. We will prove strong well-posedness of that equation, continuity and monotonicity with respect to the space variable $u$ of the solution. In Paragraph I.1.2, we will deduce existence and uniqueness of a strong solution to equation (15).

\section{I.1.1 Existence, uniqueness and continuity of the diffusion}

Let $M \in \mathbb{N} \backslash\{0\}$. Recall that $\varphi$ is even and decreasing on $[0,+\infty)$. Let us define $\varphi_{M}(x):=$ $\varphi(|x| \wedge M)$. The interest in replacing $\varphi$ by $\varphi_{M}$ is that $\varphi_{M}$ is now bounded below by a positive constant: for each $x \in \mathbb{R}, \varphi_{M}(x) \geqslant \varphi(M)>0$. Let us consider the following equation

$$
\begin{aligned}
y_{t}^{M}(u)=g(u) & +\int_{0}^{t} \frac{1}{m_{s}^{M}(u)^{1 / 2}} \int_{\mathbb{R}} \cos \left(k y_{s}^{M}(u)\right) f(k) \mathrm{d} w^{\Re}(k, s) \\
& +\int_{0}^{t} \frac{1}{m_{s}^{M}(u)^{1 / 2}} \int_{\mathbb{R}} \sin \left(k y_{s}^{M}(u)\right) f(k) \mathrm{d} w^{\Im}(k, s) .
\end{aligned}
$$

where $m_{s}^{M}(u)=\int_{0}^{1} \varphi_{M}\left(y_{s}^{M}(u)-y_{s}^{M}(v)\right) \mathrm{d} v$. Since the mass function $m_{s}^{M}$ is uniformly bounded below by $\varphi(M)$, this equation is easier to resolve and we expect that the solution also satisfies equation (15) up to a certain stopping time.

Following [GM11], we give the following definition:

Definition 2. A $\left(\mathcal{G}_{t}\right)_{t \in[0, T]^{-a d a p t e d} \text { process }}\left(M_{t}\right)_{t \in[0, T]}$ is said to be an $L_{2}$-valued $\left(\mathcal{G}_{t}\right)_{t \in[0, T]^{-}}$ martingale if for each time $t \in[0, T], M_{t}$ belongs to $L_{2}([0,1], \mathbb{R})$ and $\mathbb{E}\left[\left\|M_{t}\right\|_{L_{2}}\right]<+\infty$ and if for each $h \in L_{2}([0,1], \mathbb{R})$, the scalar product $\left(M_{t}, h\right)_{L_{2}}$ is a real-valued $\left(\mathcal{G}_{t}\right)_{t \in[0, T]}$-martingale.

Recall that $\langle k\rangle:=\left(1+k^{2}\right)^{1 / 2}$. The next proposition states well-posedness for equation (16):

Proposition 3. Let $g \in L_{2+}^{\uparrow}[0,1]$. Assume that $k \mapsto\langle k\rangle f(k)$ is square integrable. There exists a unique solution $y^{M}$ in $\mathcal{C}\left([0, T], L_{2}[0,1]\right)$ to equation (16). Furthermore, the process $\left(y_{t}^{M}\right)_{t \in[0, T]}$ is an $L_{2}$-valued continuous $\left(\mathcal{G}_{t}\right)_{t \in[0, T]}$-martingale.

Remark 4. In this proposition and in every following result, we assume, at least, that $k \mapsto\langle k\rangle f(k)$ is square integrable on $\mathbb{R}$. In the particular case of $f_{\alpha}(k)=\frac{1}{\langle k\rangle^{\alpha}}=\frac{1}{\left(1+k^{2}\right)^{\alpha / 2}}$, this assumption is equivalent to the condition $\alpha>\frac{3}{2}$.

Proof. The proof is based on a fixed-point argument, very similar to Proposition 3.5 in [Mar18]. Define $\left(\mathcal{M},\|\cdot\|_{\mathcal{M}}\right)$ the space of all $z \in L_{2}\left(\Omega, C\left([0, T], L_{2}[0,1]\right)\right)$ such that $\left(z(\omega)_{t}\right)_{t \in[0, T]}$ is a $\left(\mathcal{G}_{t}\right)_{t \in[0, T]}$-adapted process with values in $L_{2}[0,1]$. The definition of $\|\cdot\|_{\mathcal{M}}$ is given by $\|z\|_{\mathcal{M}}:=$ $\mathbb{E}\left[\sup _{t \leqslant T} \int_{0}^{1}\left|z_{t}(u)\right|^{2} \mathrm{~d} u\right]^{1 / 2}$. Define

$$
\begin{aligned}
\psi(z)_{t}(u):=g(u) & +\int_{0}^{t} \frac{1}{m_{s}^{z}(u)^{1 / 2}} \int_{\mathbb{R}} \cos \left(k z_{s}(u)\right) f(k) \mathrm{d} w^{\Re}(k, s) \\
& +\int_{0}^{t} \frac{1}{m_{s}^{z}(u)^{1 / 2}} \int_{\mathbb{R}} \sin \left(k z_{s}(u)\right) f(k) \mathrm{d} w^{\Im}(k, s),
\end{aligned}
$$

where $m_{s}^{z}(u)=\int_{0}^{1} \varphi_{M}\left(z_{s}(u)-z_{s}(v)\right) \mathrm{d} v$. For each $z \in \mathcal{M}, \psi(z)$ belongs to $\mathcal{M}$, since by 
Burkholder-Davis-Gundy inequality, there is $C>0$ such that

$$
\begin{aligned}
\mathbb{E}\left[\sup _{t \leqslant T} \int_{0}^{1}\left|\psi(z)_{t}(u)\right|^{2} \mathrm{~d} u\right] \leqslant & 3\|g\|_{L_{2}}^{2}+C \mathbb{E}\left[\int_{0}^{1} \int_{0}^{T} \int_{\mathbb{R}} \frac{\cos ^{2}\left(k z_{s}(u)\right) f^{2}(k)}{m_{s}^{z}(u)} \mathrm{d} k \mathrm{~d} s \mathrm{~d} u\right] \\
& +C \mathbb{E}\left[\int_{0}^{1} \int_{0}^{T} \int_{\mathbb{R}} \frac{\sin ^{2}\left(k z_{s}(u)\right) f^{2}(k)}{m_{s}^{z}(u)} \mathrm{d} k \mathrm{~d} s \mathrm{~d} u\right] \\
\leqslant & 3\|g\|_{L_{2}}^{2}+C\|f\|_{L_{2}}^{2} \mathbb{E}\left[\int_{0}^{1} \int_{0}^{T} \frac{1}{m_{s}^{z}(u)} \mathrm{d} s \mathrm{~d} u\right] \leqslant 3\|g\|_{L_{2}}^{2}+C_{M}\|f\|_{L_{2}}^{2},
\end{aligned}
$$

because $m_{s}^{z} \geqslant \varphi(M)>0$. Moreover, $\left(\psi(z)_{t}\right)_{t \in[0, T]}$ is an $L_{2}$-valued martingale and for each $t \in[0, T]$

$$
\begin{aligned}
\mathbb{E}\left[\sup _{s \leqslant t} \int_{0}^{1}\left|\psi\left(z^{1}\right)_{s}-\psi\left(z^{2}\right)_{s}\right|^{2}(u) \mathrm{d} u\right] \\
\leqslant C \mathbb{E}\left[\int_{0}^{1} \int_{0}^{t} \int_{\mathbb{R}}\left|\frac{\cos \left(k z_{s}^{1}(u)\right) f(k)}{m_{s}^{z^{1}}(u)^{1 / 2}}-\frac{\cos \left(k z_{s}^{2}(u)\right) f(k)}{m_{s}^{z^{2}}(u)^{1 / 2}}\right|^{2} \mathrm{~d} k \mathrm{~d} s \mathrm{~d} u\right] \\
+C \mathbb{E}\left[\int_{0}^{1} \int_{0}^{t} \int_{\mathbb{R}}\left|\frac{\sin \left(k z_{s}^{1}(u)\right) f(k)}{m_{s}^{z^{1}}(u)^{1 / 2}}-\frac{\sin \left(k z_{s}^{2}(u)\right) f(k)}{m_{s}^{z^{2}}(u)^{1 / 2}}\right|^{2} \mathrm{~d} k \mathrm{~d} s \mathrm{~d} u\right] .
\end{aligned}
$$

For every $u \in[0,1]$ and every $s \in[0, T],\left|\cos \left(k z_{s}^{2}(u)\right)-\cos \left(k z_{s}^{1}(u)\right)\right| \leqslant k\left|z_{s}^{2}(u)-z_{s}^{1}(u)\right|$ and the same Lipschitz estimate holds for the sine function. Furthermore, $\varphi_{M}$ is bounded below and Lipschitz-continuous, since $\varphi_{M}$ is $\mathcal{C}^{\infty}$ on $(-M, M)$, continuous on $\mathbb{R}$ and constant on $[M,+\infty)$. Thus we have:

$$
\begin{aligned}
\left|\frac{1}{\sqrt{m_{s}^{z^{1}}(u)}}-\frac{1}{\sqrt{m_{s}^{z^{2}(u)}}}\right| & =\frac{1}{\sqrt{m_{s}^{z^{1}}(u)} \sqrt{m_{s}^{z^{2}}(u)}} \frac{1}{\sqrt{m_{s}^{z^{1}}(u)}+\sqrt{m_{s}^{z^{2}}(u)}}\left|m_{s}^{z^{1}}(u)-m_{s}^{z^{2}}(u)\right| \\
& \leqslant \frac{1}{2 \varphi(M)^{3 / 2}} \int_{0}^{1}\left|\varphi_{M}\left(z_{s}^{1}(u)-z_{s}^{1}(v)\right)-\varphi_{M}\left(z_{s}^{2}(u)-z_{s}^{2}(v)\right)\right| \mathrm{d} v \\
& \leqslant C_{M}\left(\left|z_{s}^{1}(u)-z_{s}^{2}(u)\right|+\int_{0}^{1}\left|z_{s}^{1}(v)-z_{s}^{2}(v)\right| \mathrm{d} v\right) .
\end{aligned}
$$

It follows that:

$$
\begin{aligned}
\mathbb{E}\left[\sup _{s \leqslant t} \int_{0}^{1}\left|\psi\left(z^{1}\right)_{s}-\psi\left(z^{2}\right)_{s}\right|^{2}(u) \mathrm{d} u\right] & \leqslant C_{M} \mathbb{E}\left[\int_{0}^{1} \int_{0}^{t} \int_{\mathbb{R}}\left|z_{s}^{1}(u)-z_{s}^{2}(u)\right|^{2}\left(1+|k|^{2}\right)|f(k)|^{2} \mathrm{~d} k \mathrm{~d} s \mathrm{~d} u\right] \\
& \leqslant C_{M} \int_{\mathbb{R}}\langle k\rangle^{2}|f(k)|^{2} \mathrm{~d} k \int_{0}^{t} \mathbb{E}\left[\sup _{r \leqslant s} \int_{0}^{1}\left|z_{r}^{1}(u)-z_{r}^{2}(u)\right|^{2} \mathrm{~d} u\right] \mathrm{d} s .
\end{aligned}
$$

Define $h_{n}(t):=\mathbb{E}\left[\sup _{s \leqslant t} \int_{0}^{1}\left|\psi^{\circ n}\left(z^{1}\right)_{s}-\psi^{\circ n}\left(z^{2}\right)_{s}\right|^{2}(u) \mathrm{d} u\right]$. There is a constant $C_{M, f}$ depending on $M$ and on $\int_{\mathbb{R}}\langle k\rangle^{2}|f(k)|^{2} \mathrm{~d} k$ such that for all $n \in \mathbb{N}$ and for all $t \in[0, T]$, we have $h_{n+1}(t) \leqslant$ $C_{M, f} \int_{0}^{t} h_{n}(s) \mathrm{d} s$. Therefore, $h_{n}(t) \leqslant \frac{C_{M, f}^{n} t^{n}}{n !} h_{0}(t)$ and we deduce that $\left\|\psi^{\circ n}\left(z^{1}\right)-\psi^{\circ n}\left(z^{2}\right)\right\|_{\mathcal{M}}^{2} \leqslant$ $\frac{C_{M, f} T^{n}}{n !}\left\|z^{1}-z^{2}\right\|_{\mathcal{M}}^{2}$. Let $n$ be large enough so that $\frac{C_{M, f}^{n} T^{n}}{n !}<1$, i.e. so that $\psi^{\text {on }}$ is a contraction. Then $\psi$ admits a unique fixed point, which we denote by $y^{M}$. Since $y^{M}=\psi\left(y^{M}\right)$, it is an $L_{2}$-valued continuous $\left(\mathcal{G}_{t}\right)_{t \in[0, T]}$-martingale.

In the following two propositions, we prove that the process $\left(y_{t}^{M}\right)_{t \in[0, T]}$ preserves continuity and monotonicity of the initial condition, under the same integrability assumption on $f$ than in Proposition 3. 
Proposition 5. Let $g \in L_{2+}^{\uparrow}[0,1]$ such that $g$ is $\delta$-Hölder for some $\delta>0$. Assume that $k \mapsto$ $\langle k\rangle f(k)$ is square integrable. There exists a version of $y^{M}$ in $\mathcal{C}([0,1] \times[0, T])$.

Proof. Let $u_{1}, u_{2} \in[0,1]$. Let $p \geqslant 2$ such that $p>\frac{1}{\delta}$. For every $t \in[0, T]$, by Burkholder-DavisGundy inequality,

$$
\begin{aligned}
\mathbb{E}\left[\sup _{s \leqslant t} \mid y_{s}^{M}\left(u_{1}\right)\right. & \left.-\left.y_{s}^{M}\left(u_{2}\right)\right|^{p}\right] \\
& \leqslant C_{p}\left|g\left(u_{1}\right)-g\left(u_{2}\right)\right|^{p}+C_{p, M} \mathbb{E}\left[\left(\int_{0}^{t} \int_{\mathbb{R}}\langle k\rangle^{2} f(k)^{2} \mathrm{~d} k\left|y_{s}^{M}\left(u_{1}\right)-y_{s}^{M}\left(u_{2}\right)\right|^{2} \mathrm{~d} s\right)^{\frac{p}{2}}\right] .
\end{aligned}
$$

It follows that

$$
\begin{aligned}
\mathbb{E}\left[\sup _{s \leqslant t}\left|y_{s}^{M}\left(u_{1}\right)-y_{s}^{M}\left(u_{2}\right)\right|^{p}\right] & \\
& \leqslant C_{p}\left|g\left(u_{1}\right)-g\left(u_{2}\right)\right|^{p}+C_{p, M, f} t^{p / 2-1} \mathbb{E}\left[\int_{0}^{t}\left|y_{s}^{M}\left(u_{1}\right)-y_{s}^{M}\left(u_{2}\right)\right|^{p} \mathrm{~d} s\right] \\
& \leqslant C_{p}\left|g\left(u_{1}\right)-g\left(u_{2}\right)\right|^{p}+C_{p, M, f} t^{p / 2-1} \int_{0}^{t} \mathbb{E}\left[\sup _{r \leqslant s}\left|y_{r}^{M}\left(u_{1}\right)-y_{r}^{M}\left(u_{2}\right)\right|^{p}\right] \mathrm{d} s .
\end{aligned}
$$

By Gronwall's Lemma, and using the $\delta$-Hölder regularity of $g$, we have:

$$
\mathbb{E}\left[\sup _{t \leqslant T}\left|y_{t}^{M}\left(u_{1}\right)-y_{t}^{M}\left(u_{2}\right)\right|^{p}\right] \leqslant C_{M, p, f}\left|u_{1}-u_{2}\right|^{p \delta} .
$$

Remark that $p \delta-1>0$. Let us apply Kolmogorov's Lemma (e.g in [RY99, Theorem I.2.1, p.26] with $d=1, \gamma=p$ and $\varepsilon=p \delta-1)$. Thus there exists a version $\widetilde{y}^{M}$ of $y^{M}$ in $\mathcal{C}([0,1] \times[0, T])$.

Proposition 6. Let $g \in L_{2+}^{\uparrow}[0,1]$. Let $u_{1}<u_{2} \in[0,1]$ be such that $g\left(u_{1}\right)<g\left(u_{2}\right)$. Assume that $k \mapsto\langle k\rangle f(k)$ is square integrable. Let $y^{M}$ be the solution to equation (16). Then almost surely and for every $t \in[0, T], y_{t}^{M}\left(u_{1}\right)<y_{t}^{M}\left(u_{2}\right)$.

Proof. Let $u_{1}<u_{2} \in[0,1]$ be such that $g\left(u_{1}\right)<g\left(u_{2}\right)$. Thus the process $Y_{t}=y_{t}^{M}\left(u_{2}\right)-y_{t}^{M}\left(u_{1}\right)$ satisfies

$$
Y_{t}=g\left(u_{2}\right)-g\left(u_{1}\right)+\int_{0}^{t} Y_{s} \mathrm{~d} N_{s}^{M}
$$

where we denote

$$
\begin{aligned}
N_{t}^{M}= & \int_{0}^{t} \int_{\mathbb{R}} \mathbb{1}_{\left\{y_{s}^{M}\left(u_{1}\right) \neq y_{s}^{M}\left(u_{2}\right)\right\}} \frac{\theta_{M}^{\Re}\left(y_{s}^{M}\left(u_{2}\right), k, s\right)-\theta_{M}^{\Re}\left(y_{s}^{M}\left(u_{1}\right), k, s\right)}{y_{s}^{M}\left(u_{2}\right)-y_{s}^{M}\left(u_{1}\right)} f(k) \mathrm{d} w^{\Re}(k, s) \\
& +\int_{0}^{t} \int_{\mathbb{R}} \mathbb{1}_{\left\{y_{s}^{M}\left(u_{1}\right) \neq y_{s}^{M}\left(u_{2}\right)\right\}} \frac{\theta_{M}^{\Im}\left(y_{s}^{M}\left(u_{2}\right), k, s\right)-\theta_{M}^{\Im}\left(y_{s}^{M}\left(u_{1}\right), k, s\right)}{y_{s}^{M}\left(u_{2}\right)-y_{s}^{M}\left(u_{1}\right)} f(k) \mathrm{d} w^{\Im}(k, s)
\end{aligned}
$$

and $\theta_{M}^{\Re}(x, k, s)=\frac{\cos (k x)}{\left(\int_{0}^{1} \varphi_{M}\left(x-y_{s}^{M}(v)\right) \mathrm{d} v\right)^{1 / 2}}$ and $\theta_{M}^{\Im}(x, k, s)=\frac{\sin (k x)}{\left(\int_{0}^{1} \varphi_{M}\left(x-y_{s}^{M}(v)\right) \mathrm{d} v\right)^{1 / 2}}$. Thus we have

$$
\begin{aligned}
\left\langle N^{M}, N^{M}\right\rangle_{t}= & \int_{0}^{t} \int_{\mathbb{R}} \mathbb{1}_{\left\{y_{s}^{M}\left(u_{1}\right) \neq y_{s}^{M}\left(u_{2}\right)\right\}}\left|\frac{\theta_{M}^{\Re}\left(y_{s}^{M}\left(u_{2}\right), k, s\right)-\theta_{M}^{\Re}\left(y_{s}^{M}\left(u_{1}\right), k, s\right)}{y_{s}^{M}\left(u_{2}\right)-y_{s}^{M}\left(u_{1}\right)}\right|^{2} f(k)^{2} \mathrm{~d} k \mathrm{~d} s \\
& +\int_{0}^{t} \int_{\mathbb{R}} \mathbb{1}_{\left\{y_{s}^{M}\left(u_{1}\right) \neq y_{s}^{M}\left(u_{2}\right)\right\}}\left|\frac{\theta_{M}^{\Im}\left(y_{s}^{M}\left(u_{2}\right), k, s\right)-\theta_{M}^{\Im}\left(y_{s}^{M}\left(u_{1}\right), k, s\right)}{y_{s}^{M}\left(u_{2}\right)-y_{s}^{M}\left(u_{1}\right)}\right|^{2} f(k)^{2} \mathrm{~d} k \mathrm{~d} s .
\end{aligned}
$$


For every $x_{1}, x_{2} \in \mathbb{R}$, for every $k \in \mathbb{R}$ and for every $s \in[0, T]$, we have the following two estimates:

$$
\begin{aligned}
\left|\cos \left(k x_{2}\right)-\cos \left(k x_{1}\right)\right| & \leqslant k\left|x_{2}-x_{1}\right| \\
\left|\int_{0}^{1} \varphi_{M}\left(x_{2}-y_{s}^{M}(v)\right) \mathrm{d} v-\int_{0}^{1} \varphi_{M}\left(x_{1}-y_{s}^{M}(v)\right) \mathrm{d} v\right| & \leqslant \operatorname{Lip}\left(\varphi_{M}\right)\left|x_{2}-x_{1}\right| .
\end{aligned}
$$

It follows, by the same computation as (17), that

$$
\left|\frac{1}{\left(\int_{0}^{1} \varphi_{M}\left(x_{2}-y_{s}^{M}(v)\right) \mathrm{d} v\right)^{1 / 2}}-\frac{1}{\left(\int_{0}^{1} \varphi_{M}\left(x_{1}-y_{s}^{M}(v)\right) \mathrm{d} v\right)^{1 / 2}}\right| \leqslant C_{M}\left|x_{2}-x_{1}\right| .
$$

Thus for every $x_{1}, x_{2} \in \mathbb{R}$,

$$
\begin{aligned}
\mid \theta_{M}^{\Re}\left(x_{2}, k, s\right)-\theta_{M}^{\Re} & \left(x_{1}, k, s\right) \mid \\
\leqslant & \left|\frac{\cos \left(k x_{2}\right)-\cos \left(k x_{1}\right)}{\left(\int_{0}^{1} \varphi_{M}\left(x_{2}-y_{s}^{M}(v)\right) \mathrm{d} v\right)^{1 / 2}}\right| \\
& +\left|\cos \left(k x_{1}\right)\right| \frac{1}{\left(\int_{0}^{1} \varphi_{M}\left(x_{2}-y_{s}^{M}(v)\right) \mathrm{d} v\right)^{1 / 2}}-\frac{1}{\left(\int_{0}^{1} \varphi_{M}\left(x_{1}-y_{s}^{M}(v)\right) \mathrm{d} v\right)^{1 / 2}} \mid \\
\leqslant & C_{M}\langle k\rangle\left|x_{2}-x_{1}\right|,
\end{aligned}
$$

Therefore, for every $s \in[0, T]$,

$$
\mathbb{1}_{\left\{y_{s}^{M}\left(u_{1}\right) \neq y_{s}^{M}\left(u_{2}\right)\right\}}\left|\frac{\theta_{M}^{\Re}\left(y_{s}^{M}\left(u_{2}\right), k, s\right)-\theta_{M}^{\Re}\left(y_{s}^{M}\left(u_{1}\right), k, s\right)}{y_{s}^{M}\left(u_{2}\right)-y_{s}^{M}\left(u_{1}\right)}\right|^{2} \leqslant C_{M}\langle k\rangle^{2} .
$$

We have a similar bound on $\theta^{\Im}$. We deduce that $\frac{\mathrm{d}\left\langle N^{M}, N^{M}\right\rangle_{s}}{\mathrm{~d} s} \leqslant C_{M} \int_{\mathbb{R}}\langle k\rangle^{2} f(k)^{2} \mathrm{~d} k$ for each $s \in[0, T]$. Hence the stochastic differential equation (18) has a unique solution and it is $Y_{t}=$ $\left(g\left(u_{2}\right)-g\left(u_{1}\right)\right) \exp \left(N_{t}^{M}-\frac{1}{2}\left\langle N^{M}, N^{M}\right\rangle_{t}\right)$. In particular

$$
y_{t}^{M}\left(u_{2}\right)-y_{t}^{M}\left(u_{1}\right)=\left(g\left(u_{2}\right)-g\left(u_{1}\right)\right) \exp \left(N_{t}^{M}-\frac{1}{2}\left\langle N^{M}, N^{M}\right\rangle_{t}\right) .
$$

Since $g\left(u_{1}\right)<g\left(u_{2}\right)$, we deduce that for every $t \in[0, T], Y_{t}>0$. Thus for every $t \in[0, T]$, $y_{t}^{M}\left(u_{1}\right)<y_{t}^{M}\left(u_{2}\right)$.

Corollary 7. Let $g \in L_{2+}^{\uparrow}[0,1]$ such that $g$ is $\delta$-Hölder for some $\delta>\frac{1}{2}$. Assume that $k \mapsto\langle k\rangle f(k)$ is square integrable. Then there is a version $y^{M}$ of the solution to equation $(16)$ in $\mathcal{C}([0,1] \times[0, T])$ such that almost surely, for each $t \in[0, T], u \in[0,1] \mapsto y_{t}^{M}(u)$ is strictly increasing.

Proof. By Proposition 5, we know that there is a version $y^{M}$ of the solution to (16) jointly continuous in time and space.

Furthermore, by Proposition 6 , there exists an almost sure event $\widetilde{\Omega}$ under which $y^{M}$ belongs to $\mathcal{C}([0,1] \times[0, T])$ and for every $t \in[0, T]$ and for every $u_{1}, u_{2} \in \mathbb{Q} \cap[0,1]$ such that $u_{1}<u_{2}$, we have $y_{t}^{M}\left(u_{1}\right)<y_{t}^{M}\left(u_{2}\right)$. Since $u \mapsto y_{t}^{M}(u)$ is continuous under the event $\widetilde{\Omega}$, we deduce that $y_{t}^{M}\left(u_{1}\right)<y_{t}^{M}\left(u_{2}\right)$ holds with every $u_{1}<u_{2} \in[0,1]$. 


\section{I.1.2 Construction of a non-blowing solution on the global time interval $[0, T]$}

In this paragraph, we build a solution to equation (15), provided that the initial condition $g$ is smooth enough.

Definition 8. Let $\mathbf{G}^{1}$ denote the set of $\mathcal{C}^{1}$-functions $g:[0,1] \rightarrow \mathbb{R}$ such that for all $u<v$, $g(u)<g(v)$.

Remark that every $g$ in $\mathbf{G}^{1}$ is the quantile function of a measure $\mu_{0}$, which is absolutely continuous with respect to the Lebesgue measure on $\mathbb{R}$. Indeed, let $F_{0}$ be the inverse map of $g$, i.e. the unique map $F_{0}:[g(0), g(1)] \rightarrow[0,1]$ such that $F_{0} \circ g=\operatorname{id}_{[0,1]}$, and let $p_{0}$ the first derivative of $F_{0}$. Then $F_{0}$ and $g$ are respectively the cumulative distribution function (c.d.f) and the quantile function of the measure $\mu_{0}$ with density $p_{0}$. Furthermore, $p_{0}$ is continuous and has a compact support equal to $[g(0), g(1)]$.

Let $g \in \mathbf{G}^{1}$. Let us fix $M_{0}$ an integer such that $M_{0}>g(1)-g(0)$. We want to construct a solution to equation (15) starting at $g$, well-defined and continuous on the whole interval $[0, T]$. We will construct it on the basis of the family $\left(y^{M}\right)_{M \geqslant M_{0}}$ of solutions to equation (16) for each $M \geqslant M_{0}$. Since $g$ belongs to $\mathbf{G}^{1}$, the assumptions made in Propositions 3 and 5 and Corollary 7 can be applied. Thus for every $u, v \in[0,1]$ and for every $t \in[0, T],\left|y_{t}^{M}(u)-y_{t}^{M}(v)\right| \leqslant$ $y_{t}^{M}(1)-y_{t}^{M}(0)$. For every $M, M^{\prime} \geqslant M_{0}$, define

$$
\tau_{M}\left(y^{M^{\prime}}\right):=\inf \left\{t \geqslant 0: y_{t}^{M^{\prime}}(1)-y_{t}^{M^{\prime}}(0) \geqslant M\right\} \wedge T .
$$

Since $M>g(1)-g(0)$ and since the process $y^{M^{\prime}}(1)-y^{M^{\prime}}(0)$ is continuous, $\tau_{M}\left(y^{M^{\prime}}\right)>0$ almost surely for every $M, M^{\prime} \geqslant M_{0}$. Assume that $M \leqslant M^{\prime}$. Then for every $s \leqslant \tau_{M}\left(y^{M^{\prime}}\right)$, for every $u, v \in[0,1],\left|y_{s}^{M^{\prime}}(u)-y_{s}^{M^{\prime}}(v)\right| \leqslant M \leqslant M^{\prime}$ and thus

$$
\varphi_{M^{\prime}}\left(y_{s}^{M^{\prime}}(u)-y_{s}^{M^{\prime}}(v)\right)=\varphi\left(y_{s}^{M^{\prime}}(u)-y_{s}^{M^{\prime}}(v)\right)=\varphi_{M}\left(y_{s}^{M^{\prime}}(u)-y_{s}^{M^{\prime}}(v)\right) .
$$

Let $\sigma=\tau_{M}\left(y^{M}\right) \wedge \tau_{M}\left(y^{M^{\prime}}\right)$. We deduce from the latter equality that the processes $\left(y_{t \wedge \sigma}^{M}\right)_{t \in[0, T]}$ and $\left(y_{t \wedge \sigma}^{M^{\prime}}\right)_{t \in[0, T]}$ are both solutions to the same stochastic differential equation:

$$
z_{t}(u)=g(u)+\int_{0}^{t \wedge \sigma} \frac{1}{m_{s}^{z}(u)^{1 / 2}}\left(\int_{\mathbb{R}} \cos \left(k z_{s}(u)\right) f(k) \mathrm{d} w^{\Re}(k, s)+\int_{\mathbb{R}} \sin \left(k z_{s}(u)\right) f(k) \mathrm{d} w^{\Im}(k, s)\right),
$$

where $m_{s}^{z}(u)=\int_{0}^{1} \varphi_{M}\left(z_{s}(u)-z_{s}(v)\right) \mathrm{d} v$.

Assume that $k \mapsto\langle k\rangle f(k)$ is square integrable. Therefore, by pathwise uniqueness of the solution to equation (19), which follows from the same argument as in Proposition 3, we have for all $u \in[0,1]$, for all $t \in[0, T], y_{t \wedge \sigma}^{M}(u)=y_{t \wedge \sigma}^{M^{\prime}}(u)$, whence $\tau_{M}\left(y^{M}\right)=\tau_{M}\left(y^{M^{\prime}}\right)$. From now on, we will denote that stopping time by $\tau_{M}$. The sequence of stopping times $\left(\tau_{M}\right)_{M \geqslant 1}$ is non-decreasing.

Setting $\tau_{M_{0}-1}=0$, we define $y_{t}(u):=\mathbb{1}_{\{t=0\}} g(u)+\sum_{M=M_{0}}^{+\infty} \mathbb{1}_{\left\{t \in\left(\tau_{M-1}, \tau_{M}\right]\right\}} y_{t}^{M}(u)$ for every $t \in[0, T]$ and $u \in[0,1]$. Let $\tau_{\infty}:=\sup _{M \geqslant M_{0}} \tau_{M}$. Clearly, $\tau_{\infty}>0$ almost surely. Since $\tau_{M} \leqslant T$ for every $M \geqslant M_{0}$, we have $\tau_{\infty} \leqslant T$. Furthermore, for each $M \geqslant M_{0}, y=y^{M}$ on $\left[0, \tau_{M}\right]$ and on the interval $\left[0, \tau_{\infty}\right),\left(y_{t}\right)_{t \in[0, T]}$ is solution to equation (15).

Let us remark that $\mathbb{P}$-almost surely, $u \mapsto y_{t}(u)$ is strictly increasing for every $t \in[0, T]$. Moreover, the following proposition states that it is the unique solution in $\mathcal{C}([0,1] \times[0, T])$ to equation (15).

Proposition 9. Let $g \in \mathbf{G}^{1}$. Assume that $k \mapsto\langle k\rangle f(k)$ is square integrable. There exists a unique solution $y$ in $\mathcal{C}([0,1] \times[0, T])$ to equation (15) and this solution is defined on $[0, T]$. Furthermore, the process $\left(y_{t}\right)_{t \in[0, T]}$ is $\left(\mathcal{G}_{t}\right)_{t \in[0, T]}$-adapted. 
Proof. First, we prove that $\tau_{\infty}$ defined above is almost surely equal to $T$. Let $M \geqslant M_{0}$. Let us estimate $\mathbb{P}\left[\tau_{M}<T\right]$. Define $z_{t}^{M}:=y_{t}^{M}(1)-y_{t}^{M}(0)$. Then $\left(z_{t}^{M}\right)_{t \in[0, T]}$ is a continuous and square integrable local martingale on $[0, T]$ and thus there is a standard $\mathbb{P}$-Brownian motion $\beta$ such that $z_{t}^{M}=g(1)-g(0)+\beta_{\left\langle z^{M}, z^{M}\right\rangle_{t}}$. Moreover, $\tau_{M}=\inf \left\{t: z_{t}^{M} \geqslant M\right\} \wedge T$. Under the event $\left\{\tau_{M}<T\right\}$, there is a random time $t_{0} \in[0, T)$ such that $z_{t_{0}}^{M} \geqslant M$ whereas for all $t \in[0, T]$, $z_{t}^{M}>0$ by Proposition 6 . Let us define the process $\left(\gamma_{t}\right)_{t \geqslant 0}$ by $\gamma_{t}:=g(1)-g(0)+\beta_{t}$. Under the measure $\mathbb{P}$, it is a Brownian motion starting at $g(1)-g(0) \in(0, M)$. Moreover, under the event $\left\{\tau_{M}<T\right\},\left(\gamma_{t}\right)_{t \geqslant 0}$ reaches the level $M$ before it reaches the level 0 . Therefore, $\mathbb{P}\left[\tau_{M}<T\right] \leqslant$ $\mathbb{P}\left[\left(\gamma_{t}\right)_{t \geqslant 0}\right.$ reaches $M$ before 0$]=\frac{g(1)-g(0)}{M}$. Since $\left\{\tau_{M}<T\right\}_{M \geqslant M_{0}}$ is a non-increasing sequence of events, we deduce that $\mathbb{P}\left[\bigcap_{\left\{M \geqslant M_{0}\right\}}\left\{\tau_{M}<T\right\}\right]=0$. Thus $\mathbb{P}$-almost surely, there exists $M \geqslant M_{0}$ such that $\tau_{M}=T$, whence $y=y^{M}$. It follows that $\tau_{\infty}=T$ almost surely. Thus $y$ is a continuous solution to equation (15) defined on $[0, T]$.

Let us now prove pathwise uniqueness. Let $x^{1}$ and $x^{2}$ be two solutions on $(\Omega, \mathcal{G}, \mathbb{P})$ to equation (15) in $\mathcal{C}([0,1] \times[0, T])$. Let $\varepsilon>0$. For every $M \geqslant M_{0}$, let us define the following event: $A_{M}^{i}:=\left\{\omega \in \Omega: \sup _{u \in[0,1], t \in[0, T]}\left|x_{t}^{i}(u)\right|(\omega) \leqslant \frac{M}{2}\right\}, i=1,2$. Let $A_{M}:=A_{M}^{1} \cap A_{M}^{2}$. The sequence of events $\left(A_{M}\right)_{M \geqslant M_{0}}$ is non-decreasing and it follows from the fact that $x^{1}$ and $x^{2}$ are continuous that $\mathbb{P}\left[\bigcup_{M \geqslant M_{0}} A_{M}\right]=1$. Thus there is $M$ such that $\mathbb{P}\left[A_{M}\right]>1-\varepsilon$.

Let $M$ be such that $\mathbb{P}\left[A_{M}\right]>1-\varepsilon$. Let $\tau_{M}^{i}:=\inf \left\{t \geqslant 0: x_{t}^{i}(1)-x_{t}^{i}(0) \geqslant M\right\} \wedge T$ and $\tau_{M}=\tau_{M}^{1} \wedge \tau_{M}^{2}$. For $i=1,2$, the same argument as the one given in Corollary 7 implies that almost surely for each $t \in[0, T], u \mapsto x_{t \wedge \tau_{M}}^{i}(u)$ is strictly increasing. Therefore, under the event $A_{M}$, the equality $\tau_{M}=T$ holds. Moreover, the processes $\left(x_{t \wedge \tau_{M}}^{1}\right)_{t \in[0, T]}$ and $\left(x_{t \wedge \tau_{M}}^{2}\right)_{t \in[0, T]}$ satisfy equation (16) up to the same stopping time $\tau_{M}$. By Proposition 3, pathwise uniqueness holds for equation (16), so $\mathbb{P}\left[x_{\cdot \wedge \tau_{M}}^{1} \neq x_{\cdot \wedge \tau_{M}}^{2}\right]=0$. In particular, $0=\mathbb{P}\left[\left\{x_{\cdot \wedge \tau_{M}}^{1} \neq x_{\cdot \wedge \tau_{M}}^{2}\right\} \cap A_{M}\right]=$ $\mathbb{P}\left[\left\{x^{1} \neq x^{2}\right\} \cap A_{M}\right]$. It follows that $\mathbb{P}\left[x^{1} \neq x^{2}\right]<\varepsilon$ for every $\varepsilon>0$. Since $\varepsilon>0$ is arbitrary, we conclude that $\mathbb{P}\left[x^{1} \neq x^{2}\right]=0$ and pathwise uniqueness holds for (15).

\section{I.1.3 Higher regularity of the solution map}

Let us remark that there is a strong relation between the regularity, for each fixed $t$, of the map $u \mapsto y_{t}(u)$ and the rate of decay at infinity of $f$. We have already seen in Proposition 9 that the afore-mentioned map is continuous for every $t \in[0, T]$ if $k \mapsto\langle k\rangle f(k)$ belongs to $L_{2}(\mathbb{R})$. By differentiating formally $y$ with respect to $u$, we expect that the derivative of $y$ is a solution to the following linear stochastic differential equation for every $u \in[0,1]$ :

$$
z_{t}(u)=g^{\prime}(u)+\int_{0}^{t} z_{s}(u) \int_{\mathbb{R}} \phi^{\Re}(u, k, s) f(k) \mathrm{d} w^{\Re}(k, s)+\int_{0}^{t} z_{s}(u) \int_{\mathbb{R}} \phi^{\Im}(u, k, s) f(k) \mathrm{d} w^{\Im}(k, s),
$$

where

$$
\begin{aligned}
\phi^{\Re}(u, k, s) & :=\frac{-k \sin \left(k y_{s}(u)\right)}{\left(\int_{0}^{1} \varphi\left(y_{s}(u)-y_{s}(v)\right) \mathrm{d} v\right)^{1 / 2}}-\frac{\cos \left(k y_{s}(u)\right) \int_{0}^{1} \varphi^{\prime}\left(y_{s}(u)-y_{s}(v)\right) \mathrm{d} v}{2\left(\int_{0}^{1} \varphi\left(y_{s}(u)-y_{s}(v)\right) \mathrm{d} v\right)^{3 / 2}} ; \\
\phi^{\Im}(u, k, s) & :=\frac{k \cos \left(k y_{s}(u)\right)}{\left(\int_{0}^{1} \varphi\left(y_{s}(u)-y_{s}(v)\right) \mathrm{d} v\right)^{1 / 2}}-\frac{\sin \left(k y_{s}(u)\right) \int_{0}^{1} \varphi^{\prime}\left(y_{s}(u)-y_{s}(v)\right) \mathrm{d} v}{2\left(\int_{0}^{1} \varphi\left(y_{s}(u)-y_{s}(v)\right) \mathrm{d} v\right)^{3 / 2}} .
\end{aligned}
$$

For every $j \in \mathbb{N}$ and every $\theta \in[0,1)$, let $\mathbf{G}^{j+\theta}$ denote the set of functions $g \in \mathbf{G}^{1}$ which are $j$-times differentiable and such that $g^{(j)}$ is $\theta$-Hölder continuous.

Proposition 10. Let $\theta \in(0,1)$. Let $g \in \mathbf{G}^{1+\theta}$. Assume that $k \mapsto\langle k\rangle^{1+\theta} f(k)$ is square integrable. Almost surely, for every $t \in[0, T]$, the map $u \mapsto y_{t}(u)$ belongs to $\mathbf{G}^{1+\theta^{\prime}}$ for every $0 \leqslant \theta^{\prime}<\theta$ and $\left(\partial_{u} y_{t}\right)_{t \in[0, T]}$ satisfies equation (20). Moreover, the derivative has the following 
explicit form:

$$
\begin{aligned}
\partial_{u} y_{t}(u)=g^{\prime}(u) \exp \left(\int_{0}^{t} \int_{\mathbb{R}} \phi^{\Re}(u, k, s) f(k) \mathrm{d} w^{\Re}(k, s)+\int_{0}^{t} \int_{\mathbb{R}} \phi^{\Im}(u, k, s) f(k) \mathrm{d} w^{\Im}(k, s)\right. \\
\left.-\frac{1}{2} \int_{0}^{t} \int_{\mathbb{R}}\left(\phi^{\Re}(u, k, s)^{2}+\phi^{\Im}(u, k, s)^{2}\right) f(k)^{2} \mathrm{~d} k \mathrm{~d} s\right) .
\end{aligned}
$$

More generally, if for an integer $j \geqslant 1, g$ belongs to $\mathbf{G}^{j+\theta}$ and $k \mapsto\langle k\rangle^{j+\theta} f(k)$ is square integrable, then almost surely, for every $t \in[0, T]$, the map $u \mapsto y_{t}(u)$ belongs to $\mathbf{G}^{j+\theta^{\prime}}$ for every $0 \leqslant \theta^{\prime}<\theta$.

Remark 11. Let us consider the case of $f_{\alpha}(k)=\frac{1}{\langle k\rangle^{\alpha}}$. The assumption $\langle k\rangle^{j+\theta} f_{\alpha}(k) \in L_{2}(\mathbb{R})$ is equivalent to the condition $\alpha>j+\theta+\frac{1}{2}$. If $f$ is the Cauchy density $f(k)=\frac{1}{1+k^{2}}$, then the process $u \mapsto y_{t}(u)$ is differentiable and its derivative is $\theta^{\prime}$-Hölder continuous for every $\theta^{\prime}<\frac{1}{2}$.

By the property of monotonicity of $y_{t}$, we deduce that almost surely, for every $t \in[0, T]$, $u \mapsto \partial_{u} y_{t}(u)>0$. Recall that the c.d.f. $F_{t}$ associated to $y_{t}$ is equal to $F_{t}=\left(y_{t}\right)^{-1}$ and that the density of $p_{t}$ is the first derivative of $F_{t}$. Therefore, for every $u \in[0,1], F_{t}\left(y_{t}(u)\right)=u$ and $p_{t}\left(y_{t}(u)\right) \partial_{u} y_{t}(u)=1$. Thus for every $x \in\left[y_{t}(0), y_{t}(1)\right]$,

$$
p_{t}(x)=\frac{1}{\partial_{u} y_{t}\left(F_{t}(x)\right)} .
$$

It follows that $p_{t}$ has the same regularity than $\partial_{u} y_{t}$. If $f(k)=\frac{1}{1+k^{2}}$, then $p_{t}$ is $\theta^{\prime}$-Hölder continuous for every $\theta^{\prime}<\frac{1}{2}$.

In order to prove Proposition 10, we first replace $\varphi$ by a function $\varphi_{M}$ bounded below as previously and prove the result for the corresponding equation: since every coefficient is now Lipschitz-continuous, the computations are classical. Finally, we recover the result of Proposition 10 for a non-truncated function $\varphi$ by using the fact that almost surely for each time $t$, the solution $y$ is equal to $y \cdot \wedge \tau_{M}$ for $M$ large enough. The interest reader may find a comprehensive proof of Proposition 10 in [Mar19, Prop. II.9].

\section{I.2 Well-posedness of a perturbed Fokker-Planck equation}

We denote by $\mathcal{F} \phi$ the Fourier transform of a function $\phi$; if $\phi$ belongs to $L_{1}(\mathbb{R}), \mathcal{F} \phi(x)=$ $\frac{1}{\sqrt{2 \pi}} \int_{\mathbb{R}} e^{-i x y} \phi(y) \mathrm{d} y$. Recall that Plancherel's formula states that: $\|\phi\|_{L_{2}}=\|\mathcal{F} \phi\|_{L_{2}}$. We denote by $\mathcal{F}^{-1}$ the inverse Fourier transform.

Let us recall equation (13):

$$
\left\{\begin{aligned}
d y_{t}(u) & =b\left(y_{t}(u), \mu_{t}\right) \mathrm{d} t+\frac{1}{m_{t}(u)^{1 / 2}} \int_{\mathbb{R}} f(k) \Re\left(e^{-i k y_{t}(u)} \mathrm{d} w(k, t)\right) \\
\mu_{t} & =\operatorname{Leb}_{[0,1]} \circ y_{t}^{-1} \\
y_{0} & =g
\end{aligned}\right.
$$

with $m_{t}(u)=\int_{0}^{1} \varphi\left(y_{t}(u)-y_{t}(v)\right) \mathrm{d} v$.

In the previous section, we studied equation (13) in the case where the drift $b$ is zero. As explained in the introduction, the well-posedness of equation (13) will be deduced from the well-posedness of the diffusion without drift by a Girsanov transformation. Therefore, we will have to construct an $L_{2}$-valued process $\left(h_{t}\right)_{t \in[0, T]}$ satisfying equation (10).

Importantly, we will assume the following assumption on $b: \mathbb{R} \times \mathcal{P}_{2}(\mathbb{R}) \rightarrow \mathbb{R}$.

Definition 12. A measurable function $b: \mathbb{R} \times \mathcal{P}_{2}(\mathbb{R}) \rightarrow \mathbb{R}$ is said to satisfy the $b$-hypotheses of order $j \in \mathbb{N} \backslash\{0\}$ if: 
(B1) for every $\mu \in \mathcal{P}_{2}(\mathbb{R}), x \mapsto b(x, \mu)$ is continuous and $j$-times differentiable on $\mathbb{R}$;

(B2) for every $i \in\{0,1, \ldots, j\}$, there is a sequence $\left(C_{i}(M)\right)_{M \geqslant M_{0}}$ such that the inequality $\left|\partial_{1}^{(i)} b(x, \mu)\right| \leqslant C_{i}(M)$ holds for every $x \in \mathbb{R}$ and for every $\mu \in \mathcal{P}_{2}(\mathbb{R})$ with compact support satisfying $|\operatorname{Supp} \mu| \leqslant M$.

(B3) the sequence $\left(C_{0}(M)\right)_{M \geqslant M_{0}}$ satisfies $\frac{C_{0}(M)}{M} \underset{M \rightarrow+\infty}{\longrightarrow} 0$.

We say that $B:(x, z) \in \mathbb{R} \times L_{2}[0,1] \mapsto b\left(x, \operatorname{Leb}_{[0,1]}{ }^{\circ} z^{-1}\right)$ satisfies the $B$-hypotheses of order $j$ if the associated $b$ satisfies the $b$-hypotheses of order $j$.

Of course, every bounded function $b$ such that $(B 1)$ holds true satisfies the $b$-hypotheses of order $j$. Moreover, Definition 12 also allows us also to consider unbounded functions $b$, for which $x \mapsto b(x, \mu)$ is uniformly bounded when the support of $\mu$ is controled, in the sense of assumption (B2). Assumption (B3) is here to ensure that the solution to the drifted equation almost surely does not blow up before final time $T$, as we will explain hereafter.

Remark 13. Let us give a few examples of admissible drift functions:

- Let $b_{1}(x, \mu):=\mathbb{E}_{\mu}[a(x, Y)]=\int_{\mathbb{R}} a(x, y) \mathrm{d} \mu(y)$ or equivalently $B_{1}(x, z):=\int_{0}^{1} a(x, z(u)) \mathrm{d} u$. If $a: \mathbb{R}^{2} \rightarrow R$ is bounded and $x \mapsto a(x, y)$ is $j$-times differentiable with bounded derivatives, then $b_{1}$ satisfies the $b$-hypotheses of order $j$.

- Let $b_{2}(x, \mu):=a\left(\mathbb{E}_{\mu}[Y]\right)=a\left(\int_{\mathbb{R}} y \mathrm{~d} \mu(y)\right)$. If $a$ is bounded, then $b_{2}$ satisfies the $b$-hypotheses of every order.

- Let $b_{3}(x, \mu):=a\left(x, \mathbb{E}_{\mu}[\psi(Y)]\right)=a\left(x, \int_{\mathbb{R}} \psi(y) \mathrm{d} \mu(y)\right)$. If $a$ is bounded and $j$-times partially differentiable in its first argument with bounded derivatives and if $\psi$ is measurable, then $b_{3}$ satisfies the $b$-hypotheses of order $j$.

- Let $b_{4}(x, \mu)=a(x) \operatorname{Var}_{\mu}[Y]^{\eta}$, where $\eta<\frac{1}{2}$. If $a$ is bounded with $j$ bounded derivatives, then $b_{4}$ satisfies the $b$-hypotheses of order $j$. Indeed, if $\mu$ has a compact support with $|\operatorname{Supp} \mu| \leqslant M$, then $\operatorname{Var}[Y]^{\eta} \leqslant M^{2 \eta}$; thus $\frac{C_{0}(M)}{M} \leqslant\|a\|_{L_{\infty}} \frac{M^{2 \eta}}{M} \rightarrow 0$.

Let us emphasize the fact that, in the first example above, $\mu \mapsto b_{1}(x, \mu)$ is Lipschitz-continuous in total variation distance, with a Lipschitz constant uniform in $x$ given by the $L_{\infty}$-norm of $a$. This means that restoration of uniqueness for the Fokker-Planck equation associated with $b_{1}$ can also be obtained with finite-dimensional noise, since $b_{1}$ satisfies the assumptions of [Jou97]. Actually, Jourdain proved those results even in cases where $a$ is only bounded. Therefore, in the case of the first example above, our assumptions are more restrictive than previous existing litterature. The main interest of the study conducted here is that our result applies for examples $b_{2}, b_{3}$ and $b_{4}$, which do not satisfy the assumptions of [Jou97, MV].

The regularity assumptions on the $x$-dependence of the drift function $b$ depend on the decay rate of $f$ at infinity. Recall that the faster $f$ decays at infinity, the higher regularity we can expect on the solution process; nevertheless, the drawback is that we have to assume higher regularity on the drift function $b$ to be able to invert it. Therefore, the choice of the decay rate $\alpha$ of $f$ is crucial to obtain well-posedness for classes of drift functions of low regularity.

Definition 14. We say that $f: \mathbb{R} \rightarrow \mathbb{R}$ is of order $\alpha>0$ if there exist two constants $C$ and $c>0$ such that $\frac{c}{\langle k\rangle^{\alpha}} \leqslant f(k) \leqslant \frac{C}{\langle k\rangle^{\alpha}}$ for every $k \in \mathbb{R}$. Recall that $\langle k\rangle:=\left(1+k^{2}\right)^{1 / 2}$.

In order to make clear this relation between regularity of $b$ and decay rate of $f$, we will prove in Paragraph I.2.1 well-posedness for equation (13) in a simplified case: we will assume that the mass is constant, namely that $\varphi \equiv 1$, and that for each $\mu \in \mathcal{P}_{2}(\mathbb{R}), b(\cdot, \mu)$ belongs to a Sobolev space with a Sobolev norm uniform in $\mu$. In Paragraph I.2.2, we will then give a general statement for more general functions $\varphi$ and $b$, but the idea of proof is the same up to technicalities. 


\section{I.2.1 Simple case with constant mass and bounded drift function}

Let us assume in this paragraph that $\varphi$ is the constant function equal to one. In other words, we are studying the following equation:

$$
\left\{\begin{aligned}
d y_{t}(u) & =b\left(y_{t}(u), \mu_{t}\right) \mathrm{d} t+\int_{\mathbb{R}} f(k) \Re\left(e^{-i k y_{t}(u)} \mathrm{d} w(k, t)\right), \\
\mu_{t} & =\operatorname{Leb}_{[0,1]} \circ y_{t}^{-1} \\
y_{0} & =g
\end{aligned}\right.
$$

Let us fix $\alpha, \eta>0$ and let us assume that $f: \mathbb{R} \rightarrow \mathbb{R}$ is of order $\alpha$, according to Definition 14 . Let $b: \mathbb{R} \times \mathcal{P}_{2}(\mathbb{R}) \rightarrow \mathbb{R}$ be a measurable function such that for each $\mu \in \mathcal{P}_{2}(\mathbb{R})$, the map $x \mapsto b(x, \mu)$ belongs to the Sobolev space $H^{\eta}(\mathbb{R})$ uniformly in $\mu$, that is there is a constant $C$ such that for every $\mu \in \mathcal{P}_{2}(\mathbb{R}),\|b(\cdot, \mu)\|_{H^{\eta}} \leqslant C$, where

$$
\|\phi\|_{H^{\eta}}:=\left\|k \mapsto\langle k\rangle^{\eta} \mathcal{F} \phi(k)\right\|_{L_{2}}=\left(\int_{\mathbb{R}}\left(1+k^{2}\right)^{\eta}|\mathcal{F} \phi(k)|^{2} \mathrm{~d} k\right)^{1 / 2} .
$$

We also denote by $B: \mathbb{R} \times L_{2}[0,1] \rightarrow \mathbb{R}$ the function $B(x, z):=b\left(x, \operatorname{Leb}_{[0,1]}{ }^{\circ} z^{-1}\right)$. Of course, for every $z \in L_{2}[0,1]$, inequality $\|B(\cdot, z)\|_{H^{\eta}} \leqslant C$ also holds with the same constant $C$ as above.

The following lemma is the key step in order to apply a Girsanov transformation in equation (21). Let us fix $f, B$ and a $\left(\mathcal{G}_{t}\right)_{t \in[0, T]}$-adapted process $\left(x_{t}\right)_{t \in[0, T]}$ with values in $\mathcal{C}([0,1], \mathbb{R})$. Then we are looking for an $L_{2}(\mathbb{R}, \mathbb{C})$-valued $\left(\mathcal{G}_{t}\right)_{t \in[0, T]}$-adapted process $\left(h_{t}\right)_{t \in[0, T]}=\left(h_{t}^{\Re}+\right.$ $\left.i h_{t}^{\Im}\right)_{t \in[0, T]}$ such that for every $t \in[0, T]$ and for every $u \in[0,1]$

$$
B\left(x_{t}(u), x_{t}\right)=\int_{\mathbb{R}} e^{-i k x_{t}(u)} f(k) h_{t}(k) \mathrm{d} k,
$$

or equivalently, taking the real part of (22) (and using that $B$ and $f$ are real-valued), to find two $L_{2}(\mathbb{R}, \mathbb{R})$-valued $\left(\mathcal{G}_{t}\right)_{t \in[0, T]}$-adapted processes $\left(h_{t}^{\Re}\right)_{t \in[0, T]}$ and $\left(h_{t}^{\Im}\right)_{t \in[0, T]}$ such that

$$
B\left(x_{t}(u), x_{t}\right)=\int_{\mathbb{R}} \cos \left(k x_{t}(u)\right) f(k) h_{t}^{\Re}(k) \mathrm{d} k+\int_{\mathbb{R}} \sin \left(k x_{t}(u)\right) f(k) h_{t}^{\Im}(k) \mathrm{d} k .
$$

Lemma 15. Let $\alpha, \eta>0$. Let $f$ be of order $\alpha$ and $b: \mathbb{R} \times \mathcal{P}_{2}(\mathbb{R}) \rightarrow \mathbb{R}$ be a measurable function such that for each $\mu \in \mathcal{P}_{2}(\mathbb{R}), b(\cdot, \mu)$ belongs to $H^{\eta}(\mathbb{R})$ with a uniform $H^{\eta}$-norm. Let $\left(x_{t}\right)_{t \in[0, T]}$ be a $\left(\mathcal{G}_{t}\right)_{t \in[0, T]}$-adapted process taking values in $\mathcal{C}([0,1], \mathbb{R})$.

If $\eta \geqslant \alpha$, then there is a $\left(\mathcal{G}_{t}\right)_{t \in[0, T]}$-adapted process $\left(h_{t}\right)_{t \in[0, T]}$ which is solution, for every $t \in[0, T]$, to equation (22) and such that there exists $C>0$ depending only on $b$ and $f$ for which $\int_{0}^{T} \int_{\mathbb{R}}\left|h_{t}(k)\right|^{2} \mathrm{~d} k \mathrm{~d} t \leqslant C$ holds almost surely.

Proof. By the substitution $y=x_{t}(u)$, equation (22) is equivalent to

$$
B\left(y, x_{t}\right)=\int_{\mathbb{R}} e^{-i k y} f(k) h_{t}(k) \mathrm{d} k
$$

for every $y \in \operatorname{Im}\left(x_{t}\right)$. In particular, if a process $\left(h_{t}\right)_{t \in[0, T]}$ satisfies $(23)$ for every $y \in \mathbb{R}$, then it satisfies (22) for every $u \in[0,1]$. Computing the Fourier transform on each side of equation (23), we have $\mathcal{F}\left(f h_{t}\right)=\frac{1}{\sqrt{2 \pi}} B\left(\cdot, x_{t}\right)$. Therefore, the process defined by

$$
h_{t}(k):=\frac{1}{\sqrt{2 \pi} f(k)} \mathcal{F}^{-1}\left(B\left(\cdot, x_{t}\right)\right)(k)
$$


is solution to equation (22), provided that $h_{t}$ is square integrable for every $t \in[0, T]$. Let us compute the $L_{2}$-norm of $h$ : there are $C_{1}$ and $C_{2}$ such that

$$
\begin{aligned}
\int_{0}^{T} \int_{\mathbb{R}}\left|h_{t}(k)\right|^{2} \mathrm{~d} k \mathrm{~d} t & \leqslant C_{1} \int_{0}^{T} \int_{\mathbb{R}}\langle k\rangle^{2 \alpha}\left|\mathcal{F}^{-1}\left(B\left(\cdot, x_{t}\right)\right)(k)\right|^{2} \mathrm{~d} k \mathrm{~d} t \\
& \leqslant C_{1} \int_{0}^{T} \int_{\mathbb{R}}\langle k\rangle^{2 \eta}\left|\mathcal{F}^{-1}\left(B\left(\cdot, x_{t}\right)\right)(k)\right|^{2} \mathrm{~d} k \mathrm{~d} t \\
& =C_{1} \int_{0}^{T} \int_{\mathbb{R}}\langle k\rangle^{2 \eta}\left|\mathcal{F}\left(B\left(\cdot, x_{t}\right)\right)(k)\right|^{2} \mathrm{~d} k \mathrm{~d} t=C_{1} \int_{0}^{T}\left\|B\left(\cdot, x_{t}\right)\right\|_{H^{\eta}} \mathrm{d} t \leqslant C_{2},
\end{aligned}
$$

where we used the fact that $f$ is of order $\alpha$, that $\alpha \leqslant \eta$ and that $\mathcal{F}^{-1}(\phi)(\cdot)=\mathcal{F}(\phi)(-\cdot)$ for each $\phi \in L_{2}(\mathbb{R})$.

Let us give, in accordance with [KS91], the following sense to a weak solution to SDE (21).

Definition 16. A sextuple $\left(\Omega, \mathcal{G},\left(\mathcal{G}_{t}\right)_{t \in[0, T]}, \mathbb{P}, z, w\right)$ is said to be a weak solution to equation (21) if

- $\left(\Omega, \mathcal{G},\left(\mathcal{G}_{t}\right)_{t \in[0, T]}, \mathbb{P}\right)$ is a filtered probability space satisfying usual conditions,

- $\left(z_{t}\right)_{t \in[0, T]}$ is a continuous $\left(\mathcal{G}_{t}\right)_{t \in[0, T]}$-adapted $\mathcal{C}[0,1]$-valued process,

- $w=\left(w^{\Re}, w^{\Im}\right)$, where $\left(w^{\Re}(k, t)\right)_{k \in \mathbb{R}, t \in[0, T]}$ and $\left(w^{\Im}(k, t)\right)_{k \in \mathbb{R}, t \in[0, T]}$ are two independent $\left(\mathcal{G}_{t}\right)_{t \in[0, T]}$-Brownian sheets under $\mathbb{P}$,

- $\mathbb{P}$-almost surely, for every $t \in[0, T]$,

$$
\begin{aligned}
z_{t}(u)= & g(u)+\int_{0}^{t} \int_{\mathbb{R}} \cos \left(k z_{s}(u)\right) f(k) \mathrm{d} w^{\Re}(k, s) \\
& +\int_{0}^{t} \int_{\mathbb{R}} \sin \left(k z_{s}(u)\right) f(k) \mathrm{d} w^{\Im}(k, s)+\int_{0}^{t} B\left(z_{s}(u), z_{s}\right) \mathrm{d} s,
\end{aligned}
$$

where $B(x, z):=b\left(x, \operatorname{Leb}_{[0,1]} \circ z^{-1}\right)$.

Theorem 17. Let $g \in \mathbf{G}^{1}$. Let $f$ be of order $\alpha>\frac{3}{2}$ and $b: \mathbb{R} \times \mathcal{P}_{2}(\mathbb{R}) \rightarrow \mathbb{R}$ be a measurable function such that for each $\mu \in \mathcal{P}_{2}(\mathbb{R}), b(\cdot, \mu)$ belongs to $H^{\eta}(\mathbb{R})$ with a uniform $H^{\eta}$-norm. If $\eta \geqslant \alpha$, there exists a unique weak solution to equation (21).

Moreover, if $\left(\Omega^{i}, \mathcal{G}^{i},\left(\mathcal{G}_{t}^{i}\right)_{t \in[0, T]}, \mathbb{P}^{i}, z^{i}, w^{i}\right), i=1,2$, are two weak solutions to equation (21), then the laws of $\left(z^{1}, w^{1}\right)$ and $\left(z^{2}, w^{2}\right)$ are equal in $\mathcal{C}([0,1] \times[0, T]) \times \mathcal{C}\left(\mathbb{R} \times[0, T], \mathbb{R}^{2}\right)$.

Let us remark that the Brownian sheets $w^{1}$ and $w^{2}$ are seen here as taking values in $\mathbb{R}^{2}$, by an identification of $\mathbb{R}^{2}$ with $\mathbb{C}$.

Proof (Theorem 17, existence part). Let $\left(\Omega, \mathcal{G},\left(\mathcal{G}_{t}\right)_{t \in[0, T]}, \mathbb{P}\right)$ be a filtered probability space and $(w(u, t))_{u \in[0,1], t \in[0, T]}$ be a $\left(\mathcal{G}_{t}\right)_{t \in[0, T]}$-Brownian sheet. Since $\alpha>\frac{3}{2}$, the map $k \mapsto\langle k\rangle f(k)$ is square integrable. Let us consider equation (15) with $\varphi \equiv 1$ or equivalently equation (25) with $B \equiv 0:$

$$
y_{t}(u)=g(u)+\int_{0}^{t} \int_{\mathbb{R}} \cos \left(k y_{s}(u)\right) f(k) \mathrm{d} w^{\Re}(k, s)+\int_{0}^{t} \int_{\mathbb{R}} \sin \left(k y_{s}(u)\right) f(k) \mathrm{d} w^{\Im}(k, s) .
$$

By Proposition 9, there is a unique process $\left(y_{t}\right)_{t \in[0, T]}$ satisfying equation $(26)$ for every $u \in[0,1]$. Moreover, $\left(y_{t}\right)_{t \in[0, T]}$ is a $\left(\mathcal{G}_{t}\right)_{t \in[0, T]}$-adapted process taking values in $\mathcal{C}([0,1], \mathbb{R})$. 
Therefore, by Lemma 15 , there is a process $\left(h_{t}\right)_{t \in[0, T]}=\left(h_{t}^{\Re}+i h_{t}^{\Im}\right)_{t \in[0, T]}$ with values in $L_{2}(\mathbb{R}, \mathbb{C})$ satisfying for every $t \in[0, T]$ and $u \in[0,1]$ :

$$
\begin{aligned}
B\left(y_{t}(u), y_{t}\right)= & \int_{\mathbb{R}} \cos \left(k y_{t}(u)\right) f(k) h_{t}^{\Re}(k) \mathrm{d} k \\
& +\int_{\mathbb{R}} \sin \left(k y_{t}(u)\right) f(k) h_{t}^{\Im}(k) \mathrm{d} k
\end{aligned}
$$

and such that there exists a constant $C$ such that almost surely,

$$
\int_{0}^{T} \int_{\mathbb{R}}\left|h_{t}(k)\right|^{2} \mathrm{~d} k \mathrm{~d} t \leqslant C
$$

Therefore, we can rewrite equation (26) as follows:

$$
\begin{aligned}
y_{t}(u)= & g(u)+\int_{0}^{t} \int_{\mathbb{R}} \cos \left(k y_{s}(u)\right) f(k) \mathrm{d} w^{\Re}(k, s)+\int_{0}^{t} \int_{\mathbb{R}} \sin \left(k y_{s}(u)\right) f(k) \mathrm{d} w^{\Im}(k, s) \\
& +\int_{0}^{t} B\left(y_{s}(u), y_{s}\right) \mathrm{d} s-\int_{0}^{t} \int_{\mathbb{R}} \cos \left(k y_{s}(u)\right) f(k) h_{s}^{\Re}(k) \mathrm{d} k-\int_{0}^{t} \int_{\mathbb{R}} \sin \left(k y_{s}(u)\right) f(k) h_{s}^{\Im}(k) \mathrm{d} k \\
= & g(u)+\int_{0}^{t} \int_{\mathbb{R}} \cos \left(k y_{s}(u)\right) f(k) \mathrm{d} \widetilde{w}^{\Re}(k, s)+\int_{0}^{t} \int_{\mathbb{R}} \sin \left(k y_{s}(u)\right) f(k) \mathrm{d} \widetilde{w}^{\Im}(k, s) \\
& +\int_{0}^{t} B\left(y_{s}(u), y_{s}\right) \mathrm{d} s,
\end{aligned}
$$

where we define for every $k \in \mathbb{R}$ and for every $s \in[0, T]$

$$
\begin{aligned}
& \mathrm{d} \widetilde{w}^{\Re}(k, s):=\mathrm{d} w^{\Re}(k, s)-h_{s}^{\Re}(k) \mathrm{d} k \mathrm{~d} s, \\
& \mathrm{~d} \widetilde{w}^{\Im}(k, s):=\mathrm{d} w^{\Im}(k, s)-h_{s}^{\Im}(k) \mathrm{d} k \mathrm{~d} s .
\end{aligned}
$$

Let us consider the process $\left(G_{t}\right)_{t \in[0, T]}$ defined by:

$$
G_{t}:=\exp \left(\int_{0}^{t} \int_{\mathbb{R}} h_{s}^{\Re}(k) \mathrm{d} w^{\Re}(k, s)+\int_{0}^{t} \int_{\mathbb{R}} h_{s}^{\Im}(k) \mathrm{d} w^{\Im}(k, s)-\frac{1}{2} \int_{0}^{t} \int_{\mathbb{R}}\left|h_{s}(k)\right|^{2} \mathrm{~d} k \mathrm{~d} s\right) .
$$

By (27), there is $C>0$ such that $\exp \left(\frac{1}{2} \int_{0}^{t} \int_{\mathbb{R}}\left|h_{s}(k)\right|^{2} \mathrm{~d} k \mathrm{~d} s\right) \leqslant C$ almost surely. Thus Novikov's condition holds and the process $\left(G_{t}\right)_{t \in[0, T]}$ is a $\mathbb{P}$-martingale. Let us define the probability measure $\mathbb{Q}$ by the absolutely continuous measure with respect to $\mathbb{P}$ with density $\frac{\mathrm{d} \mathbb{Q}}{\mathrm{d} \mathbb{P}}=G_{T}$. By Girsanov's Theorem, under the probability measure $\mathbb{Q},\left(\widetilde{w}^{\Re}(k, t), \widetilde{w}^{\Im}(k, t)\right)_{k \in \mathbb{R}, t \in[0, T]}$ are two independent Brownian sheets on $\mathbb{R} \times[0, T]$ and the couple $(y, \widetilde{w})$ satisfies equation (25). Thus $\left(\Omega, \mathcal{G},\left(\mathcal{G}_{t}\right)_{t \in[0, T]}, \mathbb{Q}, y, \widetilde{w}\right)$ is a weak solution of equation $(21)$.

Let us start by proving the uniqueness part of Theorem 17 in the case where the drift function $b \equiv 0$ in equation (21), namely in the case of equation (26).

Lemma 18. Let us assume that $\left(\Omega^{i}, \mathcal{G}^{i},\left(\mathcal{G}_{t}^{i}\right)_{t \in[0, T]}, \mathbb{P}^{i}, z^{i}, w^{i}\right), i=1,2$, are two weak solutions to equation (26). Then $\left(z^{1}, w^{1}\right)$ and $\left(z^{2}, w^{2}\right)$ have same law in $\mathcal{C}([0,1] \times[0, T]) \times \mathcal{C}\left(\mathbb{R} \times[0, T], \mathbb{R}^{2}\right)$.

Proof. Recall that by Proposition 9, equation (26) has a unique pathwise solution. By an infinite-dimensional version of Yamada-Watanabe result (see [KS91, Prop 5.3.20]), it implies that the law of $\left(z^{1}, w^{1}\right)$ under $\mathbb{P}^{1}$ is equal to the law of $\left(z^{2}, w^{2}\right)$ under $\mathbb{P}^{2}$.

The proof of uniqueness in law for equation (21) is based on Girsanov's Theorem. As in the proof of the existence part, we will apply Lemma 15 to the drift function $B$ and to a weak solution to equation (21). 
Proof (Theorem 17, uniqueness part). Let us consider $\left(\Omega^{i}, \mathcal{G}^{i},\left(\mathcal{G}_{t}^{i}\right)_{t \in[0, T]}, \mathbb{P}^{i}, z^{i}, w^{i}\right)$, for $i=1,2$, two weak solutions to equation $(21)$. Let $i=1$ or 2 . In particular, $\left(z_{t}^{i}\right)_{t \in[0, T]}$ is a $\left(\mathcal{G}_{t}^{i}\right)_{t \in[0, T]^{-}}$ adapted process taking values in $\mathcal{C}([0,1], \mathbb{R})$. Thus by Lemma 15 , there is a $\left(\mathcal{G}_{t}^{i}\right)_{t \in[0, T]}$-adapted process $\left(h_{t}^{i}\right)_{t \in[0, T]}$ such that $\int_{0}^{T} \int_{\mathbb{R}}\left|h_{t}^{i}(k)\right|^{2} \mathrm{~d} k \mathrm{~d} s \leqslant C$ almost surely and for all $t \in[0, T]$ and $u \in[0,1]$,

$$
B\left(z_{t}^{i}(u), z_{t}^{i}\right)=\int_{\mathbb{R}} \cos \left(k z_{t}^{i}(u)\right) f(k) h_{t}^{i, \Re}(k) \mathrm{d} k+\int_{\mathbb{R}} \sin \left(k z_{t}^{i}(u)\right) f(k) h_{t}^{i, \Im}(k) \mathrm{d} k .
$$

Furthermore, by equation (24), there is a measurable map $\mathcal{H}: \mathcal{C}[0,1] \rightarrow L_{2}(\mathbb{R}, \mathbb{C})$ such that $h_{t}^{i}=\mathcal{H}\left(z_{t}^{i}\right)$ for every $t \in[0, T]$ and for $i=1,2$. The map $\mathcal{H}$ is defined by:

$$
\mathcal{H}(\mathbf{x}): k \mapsto \frac{1}{\sqrt{2 \pi} f(k)} \mathcal{F}^{-1}(B(\cdot, \mathbf{x}))(k)
$$

for every $\mathrm{x} \in \mathcal{C}[0,1]$.

Since $\left(z_{t}^{i}\right)_{t \in[0, T]}$ is solution to equation (25), we have $\mathbb{P}^{i}$-almost surely for every $t \in[0, T]$ and for every $u \in[0,1]$ :

$$
\begin{aligned}
z_{t}^{i}(u)= & g(u)+\int_{0}^{t} \int_{\mathbb{R}} \cos \left(k z_{s}^{i}(u)\right) f(k)\left(\mathrm{d} w^{i, \Re}(k, s)+h_{s}^{i, \Re}(k) \mathrm{d} k \mathrm{~d} s\right) \\
& +\int_{0}^{t} \int_{\mathbb{R}} \sin \left(k z_{s}^{i}(u)\right) f(k)\left(\mathrm{d} w^{i, \Im}(k, s)+h_{s}^{i, \Im}(k) \mathrm{d} k \mathrm{~d} s\right) .
\end{aligned}
$$

Let us define for every $k \in \mathbb{R}$ and every $s \in[0, T]$

$$
\begin{aligned}
& \mathrm{d} \widetilde{w}^{i, \Re}(k, s):=\mathrm{d} w^{i, \Re}(k, s)+h_{s}^{i, \Re}(k) \mathrm{d} k \mathrm{~d} s, \\
& \mathrm{~d} \widetilde{w}^{i, \Im}(k, s):=\mathrm{d} w^{i, \Im}(k, s)+h_{s}^{i, \Im}(k) \mathrm{d} k \mathrm{~d} s .
\end{aligned}
$$

Let us consider the process $\left(G_{t}^{i}\right)_{t \in[0, T]}$ defined by:

$$
G_{t}^{i}:=\exp \left(-\int_{0}^{t} \int_{\mathbb{R}} h_{s}^{i, \Re}(k) \mathrm{d} w^{i, \Re}(k, s)-\int_{0}^{t} \int_{\mathbb{R}} h_{s}^{i, \Im}(k) \mathrm{d} w^{i, \Im}(k, s)-\frac{1}{2} \int_{0}^{t} \int_{\mathbb{R}}\left|h_{s}^{i}(k)\right|^{2} \mathrm{~d} k \mathrm{~d} s\right) .
$$

Novikov's condition applies because $\int_{0}^{T} \int_{\mathbb{R}}\left|h_{t}^{i}(k)\right|^{2} \mathrm{~d} k \mathrm{~d} s \leqslant C$ almost surely and the process $\left(G_{t}^{i}\right)_{t \in[0, T]}$ is a $\mathbb{P}^{i}$-martingale. We define the probability measure $\mathbb{Q}^{i}$ by the absolutely continuous measure with respect to $\mathbb{P}^{i}$ with density $\frac{\mathrm{d}^{i}}{\mathrm{~d} \mathbb{P}^{i}}=G_{T}^{i}$. By Girsanov's Theorem, under $\mathbb{Q}^{i}$, $\widetilde{w}^{i}=\left(\widetilde{w}^{i, \Re}, \widetilde{w}^{i, \Im}\right)$ is a couple of two independent Brownian sheets and $\mathbb{Q}^{i}$-almost surely, for every $t \in[0, T]$

$$
z_{t}^{i}(u)=g(u)+\int_{0}^{t} \int_{\mathbb{R}} f(k)\left(\cos \left(k z_{s}^{i}(u)\right) \mathrm{d} \widetilde{w}^{i, \Re}(k, s)+\sin \left(k z_{s}^{i}(u)\right) \mathrm{d} \widetilde{w}^{i, \Im}(k, s)\right) .
$$

Thus $\left(\Omega^{i}, \mathcal{G}^{i},\left(\mathcal{G}_{t}^{i}\right)_{t \in[0, T]}, \mathbb{Q}^{i}, z^{i}, \widetilde{w}^{i}\right)$, for $i=1,2$, are two weak solutions to equation (21) in the case where $B \equiv 0$. By Lemma 18 , it follows that for every measurable function $\psi: \mathcal{C}([0,1] \times$ $[0, T]) \times \mathcal{C}\left(\mathbb{R} \times[0, T], \mathbb{R}^{2}\right) \rightarrow \mathbb{R}$ such that $\mathbb{E}^{\mathbb{Q}^{i}}\left[\left|\psi\left(z^{i}, \widetilde{w}^{i}\right)\right|\right]<+\infty$ for $i=1,2$, we have

$$
\mathbb{E}^{\mathbb{Q}^{1}}\left[\psi\left(z^{1}, \widetilde{w}^{1}\right)\right]=\mathbb{E}^{\mathbb{Q}^{2}}\left[\psi\left(z^{2}, \widetilde{w}^{2}\right)\right] .
$$

Let $\phi: \mathcal{C}([0,1] \times[0, T]) \times \mathcal{C}\left(\mathbb{R} \times[0, T], \mathbb{R}^{2}\right) \rightarrow \mathbb{R}$ be a bounded and measurable function. We 
have

$$
\begin{aligned}
\mathbb{E}^{\mathbb{P}^{i}}\left[\phi\left(z^{i}, w^{i}\right)\right]= & \mathbb{E}^{\mathbb{Q}^{i}}\left[\phi\left(z^{i}, w^{i}\right)\left(G_{T}^{i}\right)^{-1}\right] \\
= & \mathbb{E}^{\mathbb{Q}^{i}}\left[\phi ( z ^ { i } , w ^ { i } ) \operatorname { e x p } \left(\int_{0}^{T} \int_{\mathbb{R}} h_{s}^{i, \Re}(k) \mathrm{d} w^{i, \Re}(k, s)\right.\right. \\
& \left.\left.\quad+\int_{0}^{T} \int_{\mathbb{R}} h_{s}^{i, \Im}(k) \mathrm{d} w^{i, \Im}(k, s)+\frac{1}{2} \int_{0}^{T} \int_{\mathbb{R}}\left|h_{s}^{i}(k)\right|^{2} \mathrm{~d} k \mathrm{~d} s\right)\right] \\
= & \mathbb{E}^{\mathbb{Q}^{i}}\left[\phi ( z ^ { i } , \widetilde { w } ^ { i } + \int _ { 0 } \int _ { 0 } ^ { i } h _ { s } ^ { i } ( k ) \mathrm { d } k \mathrm { d } s ) \operatorname { e x p } \left(\int_{0}^{T} \int_{\mathbb{R}} h_{s}^{i, \Re}(k) \mathrm{d} \widetilde{w}^{i, \Re}(k, s)\right.\right. \\
\quad & \left.\left.\quad+\int_{0}^{T} \int_{\mathbb{R}} h_{s}^{i, \Im}(k) \mathrm{d} \widetilde{w}^{i, \Im}(k, s)-\frac{1}{2} \int_{0}^{T} \int_{\mathbb{R}}\left|h_{s}^{i}(k)\right|^{2} \mathrm{~d} k \mathrm{~d} s\right)\right] \\
= & \mathbb{E}^{\mathbb{Q}^{i}}\left[\psi\left(z^{i}, \widetilde{w}^{i}\right)\right], \quad
\end{aligned}
$$

where $\psi: \mathcal{C}([0,1] \times[0, T]) \times \mathcal{C}\left(\mathbb{R} \times[0, T], \mathbb{R}^{2}\right) \rightarrow \mathbb{R}$ is a measurable function, because for each $t \in[0, T], h_{t}^{i}=\mathcal{H}\left(z_{t}^{i}\right)$ with $\mathcal{H}: \mathcal{C}[0,1] \rightarrow L_{2}(\mathbb{R}, \mathbb{C})$ a measurable function. By equality (28), we deduce that $\mathbb{E}^{\mathbb{P}^{1}}\left[\phi\left(z^{1}, w^{1}\right)\right]=\mathbb{E}^{\mathbb{P}^{2}}\left[\phi\left(z^{2}, w^{2}\right)\right]$. Thus $\left(z^{1}, w^{1}\right)$ and $\left(z^{2}, w^{2}\right)$ have the same law and this completes the proof of the theorem.

\section{I.2.2 General case}

In the previous paragraph, our assumptions on $b$ were rather restrictive: for instance, the inversion statement of Lemma 15 does not apply for $b \equiv 1$ because it does not belong to $H^{\eta}(\mathbb{R})$ for any positive $\eta$. In this paragraph, we explain briefly how we can extend the well-posedness result for a larger class of drift functions $b$ or general mass functions $\varphi$. Because the proofs are very similar to the particular case seen above, the statements of this paragraph will be explained shortly without the detailled proofs: the interest reader can find the complete proofs of the results stated below in [Mar19, Parag. II.4].

Let us recall that we consider $\varphi: \mathbb{R} \rightarrow \mathbb{R}$ an even $\mathcal{C}^{\infty}$-function, such that $\varphi$ is positive and decreasing on $[0,+\infty)$. For every fixed $M>0$, we define the following assumptions:

Definition 19. A process $\left(x_{t}\right)_{t \in[0, T]}$ with values in $\mathcal{C}[0,1]$ is said to satisfy the $X_{M}$-hypotheses if:

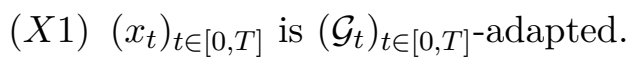

(X2) almost surely, for every $t \in[0, T], u \mapsto x_{t}(u)$ is strictly increasing.

(X3) almost surely, for every $t \in[0, T],\left|x_{t}(1)-x_{t}(0)\right| \leqslant M$.

As a consequence of Corollary 7 and of Proposition 9, the stopped process $\left(y_{t \wedge \tau_{M}}\right)_{t \in[0, T]}$ solution to the equation without drift function satisfies the assumptions of Definition 19:

Proposition 20. Let $g \in \mathbf{G}^{1}$. Assume that $f$ is of order $\alpha>\frac{3}{2}$. Let $\left(y_{t}\right)_{t \in[0, T]}$ be the unique solution to equation (15) given by Proposition 9. Let $M>g(1)-g(0)$ and recall the definition of $\tau_{M}:=\inf \left\{t \geqslant 0: y_{t}(1)-y_{t}(0) \geqslant M\right\} \wedge T$. Then $\left(y_{t \wedge \tau_{M}}\right)_{t \in[0, T]}$ satisfies the $X_{M}$-hypotheses.

Under those less restrictive assumptions on $\varphi, B$ and $x$, the following lemma shows the existence of an $L_{2}(\mathbb{R}, \mathbb{C})$-valued process $\left(h_{t}\right)_{t \in[0, T]}=\left(h_{t}^{\Re}+i h_{t}^{\Im}\right)_{t \in[0, T]}$ such that

$$
B\left(x_{t}(u), x_{t}\right)=\frac{1}{\left(\int_{0}^{1} \varphi\left(x_{t}(u)-x_{t}(v)\right) \mathrm{d} v\right)^{1 / 2}} \int_{\mathbb{R}} e^{-i k x_{t}(u)} f(k) h_{t}(k) \mathrm{d} k .
$$


Lemma 21. Let $M>g(1)-g(0), j \in \mathbb{N}$ and $\alpha>0$. Let us assume that $f$ is of order $\alpha$, that $B: \mathbb{R} \times L_{2}[0,1] \rightarrow \mathbb{R}$ satisfies the $B$-hypotheses of order $2 j$ and thatthe process $\left(x_{t}\right)_{t \in[0, T]}$ satisfies the $X_{M}$-hypotheses.

If $2 j \geqslant \alpha$, then there is a $\left(\mathcal{G}_{t}\right)_{t \in[0, T]}$-adapted process $\left(h_{t}\right)_{t \in[0, T]}$ which is solution, for every $t \in[0, T]$, to equation (30) and such that there exists $C_{M}>0$ depending only on $B, f, \varphi$ and $M$ for which $\int_{0}^{T} \int_{\mathbb{R}}\left|h_{t}(k)\right|^{2} \mathrm{~d} k \mathrm{~d} t \leqslant C_{M}$ holds almost surely.

Proof. Let $\left(x_{t}\right)_{t \in[0, T]}$ be a process satisfying the $X_{M}$-hypotheses. Therefore, for a fixed $t \in[0, T]$, the map $u \mapsto x_{t}(u)$ is a continuous strictly increasing function and can be seen as the quantile function of a measure $\mu_{t} \in \mathcal{P}_{2}(\mathbb{R})$. Let us denote by $F_{t}$ and $p_{t}$ respectively the c.d.f. and the density associated to $\mu_{t}$. More precisely, $F_{t}\left(x_{t}(u)\right)=u$ for all $u \in[0,1], F_{t}(y)=0$ for all $y \leqslant x_{t}(0)$ and $F_{t}(y)=1$ for all $y \geqslant x_{t}(1)$. Since almost surely, for every $t \in[0, T]$, $x_{t}(1)-x_{t}(0) \leqslant M$, we have $\left|\operatorname{Supp} p_{t}\right| \leqslant M$, where $\left|\operatorname{Supp} p_{t}\right|$ denotes the Lebesgue measure of the support of $p_{t}$.

By the substitution $y=x_{t}(u)$, equation (30) is equivalent to

$$
B\left(y, x_{t}\right)\left(\int_{0}^{1} \varphi\left(y-x_{t}(v)\right) \mathrm{d} v\right)^{1 / 2}=\int_{\mathbb{R}} e^{-i k y} f(k) h_{t}(k) \mathrm{d} k
$$

for every $y \in\left[x_{t}(0), x_{t}(1)\right]$. Let us fix a $\mathcal{C}^{\infty}$-function $\Psi: \mathbb{R} \rightarrow \mathbb{R}$ that is equal to 0 on $(-\infty, 0]$ and equal to 1 on $[1,+\infty)$. For every $a<b$, we define the cut-off function $\eta_{a, b}: \mathbb{R} \rightarrow \mathbb{R}$ by

$$
\eta_{a, b}(y)= \begin{cases}1 & \text { on }[a, b] \\ \Psi(y-(a-1)) & \text { on }(a-1, a) \\ \Psi(b+1-y) & \text { on }(b, b+1) \\ 0 & \text { elsewhere. }\end{cases}
$$

Let us denote by $\eta_{t}:=\eta_{x_{t}(0), x_{t}(1)}$. For every $y \in\left[x_{t}(0), x_{t}(1)\right], \eta_{t}(y)=1$. Moreover, $\eta_{t}$ has a compact support included in $\left[x_{t}(0)-1, x_{t}(1)+1\right]$. Therefore, if a process $\left(h_{t}\right)_{t \in[0, T]}$ satisfies

$$
B\left(y, x_{t}\right) \eta_{t}(y)\left(\int_{0}^{1} \varphi\left(y-x_{t}(v)\right) \mathrm{d} v\right)^{1 / 2}=\int_{\mathbb{R}} e^{-i k y} f(k) h_{t}(k) \mathrm{d} k
$$

for every $y \in \mathbb{R}$, then it satisfies (31) for every $y \in\left[x_{t}(0), x_{t}(1)\right]$ and thus it satisfies (30) for every $u \in[0,1]$. Therefore, the process defined by

$$
h_{t}(k):=\frac{1}{\sqrt{2 \pi} f(k)} \mathcal{F}^{-1}\left(B\left(\cdot, x_{t}\right) \eta_{t}\left(\int_{0}^{1} \varphi\left(\cdot-x_{t}(v)\right) \mathrm{d} v\right)^{1 / 2}\right)(k)
$$

is solution to equation (30), provided that $h_{t}$ is square integrable for every $t \in[0, T]$. Define $\Phi_{t}:=$ $B\left(\cdot, x_{t}\right) \eta_{t}\left(\int_{0}^{1} \varphi\left(\cdot-x_{t}(v)\right) \mathrm{d} v\right)^{1 / 2}$. Note that for every $t \in[0, T], y \mapsto \eta_{t}(y)\left(\int_{0}^{1} \varphi\left(y-x_{t}(v)\right) \mathrm{d} v\right)^{1 / 2}$ is a bounded $\mathcal{C}^{\infty}$-function with compact support and $y \mapsto B\left(y, x_{t}\right)$ is a bounded continuous function. Therefore, $\Phi_{t}$ belongs to $L^{1}(\mathbb{R}, \mathbb{C})$ and $h_{t}$ is well-defined. Moreover, since $\left(\Phi_{t}\right)_{t \in[0, T]}$

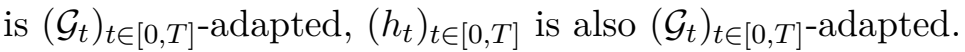

Furthermore, we know by assumption that there is $c>0$ such that for every $k \in \mathbb{R}, \frac{1}{f(k)} \leqslant$ $c\langle k\rangle^{\alpha} \leqslant c\langle k\rangle^{2 j}$. Thus by Plancherel's Theorem (and denoting by $\Delta$ the Laplacian) we have

$$
\begin{aligned}
\left\|h_{t}\right\|_{L_{2}}^{2}=\int_{\mathbb{R}}\left|h_{t}(k)\right|^{2} \mathrm{~d} k=\int_{\mathbb{R}} \frac{1}{2 \pi} \frac{\left|\mathcal{F}^{-1} \Phi_{t}(k)\right|^{2}}{|f(k)|^{2}} \mathrm{~d} k & \leqslant C \int_{\mathbb{R}}\left|\langle k\rangle^{2 j} \mathcal{F}^{-1} \Phi_{t}(k)\right|^{2} \mathrm{~d} k \\
& =C \int_{\mathbb{R}}\left|\mathcal{F}^{-1}\left((1+\Delta)^{j} \Phi_{t}\right)(k)\right|^{2} \mathrm{~d} k \\
& =C\left\|(1+\Delta)^{j} \Phi_{t}\right\|_{L_{2}}^{2} .
\end{aligned}
$$


On the one hand, $B$ satisfies the $B$-hypotheses of order $2 j$, then for every $i \in\{0,1, \ldots, 2 j\}$, for every $t \in[0, T]$ and for every $y \in \mathbb{R},\left|\partial_{1}^{(i)} B\left(y, x_{t}\right)\right| \leqslant C_{i}(M)$. On the other hand, $y \mapsto$ $\eta_{t}(y)\left(\int_{0}^{1} \varphi\left(y-x_{t}(v)\right) \mathrm{d} v\right)^{1 / 2}$ is a $\mathcal{C}^{\infty}$-function with compact support, thus this function and all its derivatives are bounded on $\mathbb{R}$. We deduce that for every $i \leqslant 2 j$, there is a constant $C_{i}$ depending on $B$ and $\varphi$ such that almost surely, for every $t \in[0, T],\left\|\partial^{i} \Phi_{t}\right\|_{L_{\infty}} \leqslant C_{i}$.

Recall that the support of $\eta_{t}$ is included in $\left[x_{t}(0)-1, x_{t}(1)+1\right]$. Henceforth, almost surely for every $t \in[0, T]$, the Lebesgue measure of the support of $\Phi_{t}$ is bounded by $M+2$. Therefore, for every $i \leqslant 2 j$, there is a constant $C_{i, M}$ such that almost surely, for every $t \in[0, T]$,

$$
\left\|\partial^{i} \Phi_{t}\right\|_{L_{2}} \leqslant\left|\operatorname{Supp} \Phi_{t}\right|^{1 / 2}\left\|\partial^{i} \Phi_{t}\right\|_{L_{\infty}} \leqslant C_{i, M}
$$

We deduce that there is $C_{M}>0$ such that $\int_{0}^{T}\left\|h_{t}\right\|_{L_{2}}^{2} \mathrm{~d} t \leqslant C_{M}$ almost surely, which completes the proof.

Thus, we can state the following theorem, which is a rewriting of Theorem 1 under the precise assumptions on $b$.

Theorem 22. Let $g \in \mathbf{G}^{1}$ and $j \in \mathbb{N} \backslash\{0\}$. Let $f$ be a function of order $\alpha>\frac{3}{2}$. Let $B$ : $\mathbb{R} \times L_{2}[0,1] \rightarrow \mathbb{R}$ satisfy the B-hypotheses of order $2 j$. If $2 j \geqslant \alpha$, there exists a weak solution to equation (13) and uniqueness in law holds for this equation.

Let us briefly explain the different steps of the proof of Theorem 22, the detailled proof being given in [Mar19, Parag. II.4].

Step 1. Let us fix $M \geqslant g(1)-g(0)$ and let us define the stopped version of equation (13):

$$
\left\{\begin{aligned}
d y_{t}(u) & =\mathbb{1}_{\left\{t \leqslant \tau_{M}\right\}}\left(B\left(y_{t}(u), y_{t}\right) \mathrm{d} t+\frac{1}{m_{t}(u)^{1 / 2}} \int_{\mathbb{R}} f(k) \Re\left(e^{-i k y_{t}(u)} \mathrm{d} w(k, t)\right)\right), \\
y_{0} & =g
\end{aligned}\right.
$$

where $m_{t}(u)=\int_{0}^{1} \varphi\left(y_{t}(u)-y_{t}(v)\right) \mathrm{d} v$ and $\tau_{M}:=\inf \left\{t \geqslant 0: y_{t}(1)-y_{t}(0) \geqslant M\right\} \wedge T$. We start by proving, for $f$ of order $\alpha>\frac{3}{2}$ and for $B$ satisfying the $B$-hypotheses of order $2 j \geqslant \alpha$, the existence of a weak solution to (32). The scheme of proof is the following: since $\alpha>\frac{3}{2}$, we know by Proposition 20 that $\left(y_{t \wedge \tau_{M}}\right)_{t \in[0, T]}$ satisfies the $X_{M}$-hypotheses. Then, by Lemma 21, there exists an appropriate process $\left(h_{t}\right)_{t \in[0, T]}$ such that $\int_{0}^{T} \int_{\mathbb{R}}\left|h_{t}(k)\right|^{2} \mathrm{~d} k \mathrm{~d} t \leqslant C_{M}$. Then the proof is the same as for Theorem 17.

Step 2. By analogy with Theorem 17 , we prove that if $\left(\Omega^{i}, \mathcal{G}^{i},\left(\mathcal{G}_{t}^{i}\right)_{t \in[0, T]}, \mathbb{P}^{i}, z^{i}, w^{i}\right), i=1,2$, are two weak solutions to equation (32), then the laws of $\left(z^{1}, w^{1}\right)$ and $\left(z^{2}, w^{2}\right)$ are equal in $\mathcal{C}([0,1] \times[0, T]) \times \mathcal{C}\left(\mathbb{R} \times[0, T], \mathbb{R}^{2}\right)$. First, we observe that this statement is true for $B \equiv 0$, by an infinite-dimensional version of Yamada-Watanabe result (see [KS91, Prop 5.3.20]). Then we show that a solution to equation (32) satisfies the $X_{M}$-hypotheses. The statement of step 2 follows by the same arguments as for Theorem 17.

Step 3. For each integer $M$ greater than $M_{0}:=g(1)-g(0)$, we consider the solution to equation (32) on the canonical probability space. Let $\Omega=\Omega_{1} \times \Omega_{2}$, where $\Omega_{1}:=\mathcal{C}([0,1] \times[0, T])$ and $\Omega_{2}:=\mathcal{C}\left(\mathbb{R} \times[0, T], \mathbb{R}^{2}\right)$, equipped with the class $\mathcal{B}(\Omega)$ of Borel subsets of $\Omega$. To every $z \in \Omega_{1}$, we associate $\zeta_{z}^{M}=\inf \left\{t \geqslant 0: z_{t}(1)-z_{t}(0) \geqslant M\right\} \wedge T$. Let $\mathcal{G}^{M}$ be the $\sigma$-algebra generated by the map $\pi^{M}: z \in \Omega_{1} \mapsto z_{\cdot \wedge \zeta_{z}^{M}} \in \Omega_{1}$. By step 1, there is a weak solution $\left(\Omega, \mathcal{G}^{M} \otimes \mathcal{B}\left(\Omega_{2}\right),\left(\mathcal{G}_{t}\right)_{t \in[0, T]}, \mathbb{Q}^{M}, z_{\cdot \wedge \zeta_{z}^{M}}, w\right)$ to equation $(32)$.

We prove that the family $\left(\mathbb{Q}^{M}\right)_{M \geqslant M_{0}}$ is consistent, which follows from uniqueness in law proved in step 2. We use Theorem V.4.2 of Parthasarathy's book [Par67, p.143] to construct a probability measure $\mathbb{Q}$ on $(\Omega, \mathcal{B}(\Omega))$ such that for each $M \geqslant M_{0}$, for each $A \in \mathcal{G}^{M}$ and for each $B \in \mathcal{B}\left(\Omega_{2}\right), \mathbb{Q}[A \times B]=\mathbb{Q}^{M}[A \times B]$. 
Step 4. In order to prove the theorem, it remains to prove that $\mathbb{Q}\left[\zeta_{z}^{M}<T\right] \rightarrow 0$ when $M \rightarrow+\infty$. We control the martingale part of a solution $\left(z_{t}\right)_{t \in[0, T]}$ to equation (13) by the same arguments as in Proposition 6. For the control of the drift part, we use assumption (B3) on $B$ (it is the only point where this assumption is needed) to obtain:

$$
\mathbb{Q}^{M}\left[\sup _{t \leqslant T}\left|\int_{0}^{t \wedge \zeta^{M}}\left(B\left(z_{s}(1), z_{s}\right)-B\left(z_{s}(0), z_{s}\right)\right) \mathrm{d} s\right| \geqslant \frac{M}{2}\right] \leqslant \frac{2}{M} 2 T C_{0}(M) \underset{M \rightarrow+\infty}{\longrightarrow} 0 .
$$

This concludes the proof of weak well-posedness for equation (13).

\section{A continuum of admissible drift functions}

In this part, we make the connection between the result of restoration of uniqueness obtained in Theorem 17 and results of existence and uniqueness for standard McKean-Vlasov equations driven by a velocity field that is merely measurable in the space variable (see [Jou97, MV, Lac18, RZ]). The connection reads in the form of a new existence and uniqueness result but for a suitable notion of weak solution and for a class of admissible drifts. We address both in the next two subsections.

\section{II.1 Description of the class of admissible drift functions}

Recall the definition of the distance in total variation between two probability measures. For any $\mu, \nu \in \mathcal{P}(\mathbb{R})$,

$$
\mathrm{d}_{\mathrm{TV}}(\mu, \nu)=2 \inf _{\substack{\mathcal{L}(X)=\mu \\ \mathcal{L}(Y)=\nu}} \mathbb{P}[X \neq Y]
$$

where the infimum is taken here over every coupling $(X, Y)$ of random variables $X$ and $Y$ in $L_{2}(\Omega, \mathcal{F}, \mathbb{P})$ with respective distributions $\mu$ and $\nu$, where $(\Omega, \mathcal{F}, \mathbb{P})$ is any fixed Polish and atomless probability space.

Let us define the following space on which we will consider the drift function:

Definition 23. Let $\eta>0$ and $\delta \in[0,1]$. We say that $b: \mathbb{R} \times \mathcal{P}_{2}(\mathbb{R}) \rightarrow \mathbb{R}$ is of class $\left(H^{\eta}, \mathcal{C}^{\delta}\right)$ if there are measurable functions $\lambda^{\Re}, \lambda^{\Im}: \mathbb{R} \times \mathcal{P}_{2}(\mathbb{R}) \rightarrow \mathbb{R}$ and $\Lambda: \mathbb{R} \rightarrow \mathbb{R}_{+}$such that for every $x \in \mathbb{R}$ and $\mu \in \mathcal{P}_{2}(\mathbb{R})$,

$$
b(x, \mu)=\int_{\mathbb{R}}\langle k\rangle^{-\eta}\left(\cos (k x) \lambda^{\Re}(k, \mu)+\sin (k x) \lambda^{\Im}(k, \mu)\right) \mathrm{d} k,
$$

where

- $\lambda:=\lambda^{\Re}+i \lambda^{\Im}$ is bounded in the measure variable: for every $k \in \mathbb{R}$ and $\mu \in \mathcal{P}_{2}(\mathbb{R})$, $|\lambda(k, \mu)| \leqslant \Lambda(k)$;

- $\lambda$ is $\delta$-Hölder continuous in the measure variable: for every $k$ and for every $\mu, \nu \in \mathcal{P}_{2}(\mathbb{R})$, $|\lambda(k, \mu)-\lambda(k, \nu)| \leqslant \Lambda(k) \mathrm{d}_{\mathrm{TV}}(\mu, \nu)^{\delta} ;$

- $\Lambda \in L_{1}(\mathbb{R}) \cap L_{2}(\mathbb{R})$.

In particular, if $b$ is of class $\left(H^{\eta}, \mathcal{C}^{\delta}\right)$, then for every $\mu \in \mathcal{P}_{2}(\mathbb{R})$, the map $x \mapsto b(x, \mu)$ belongs to the Sobolev space $H^{\eta}(\mathbb{R})$. Indeed, denoting by $\mathcal{F}(b(\cdot, \mu))$ the Fourier transform of $b(\cdot, \mu)$, we have

$$
\int_{\mathbb{R}}\left|\langle k\rangle^{\eta} \mathcal{F}(b(\cdot, \mu))(k)\right|^{2} \mathrm{~d} k \leqslant C \int_{\mathbb{R}}|\lambda(-k, \mu)|^{2} \mathrm{~d} k \leqslant C \int_{\mathbb{R}} \Lambda(-k)^{2} \mathrm{~d} k<+\infty
$$


Moreover, if $b$ is of class $\left(H^{\eta}, \mathcal{C}^{\delta}\right)$, then for every $x \in \mathbb{R}, \mu \mapsto b(x, \mu)$ is $\delta$-Hölder continuous in total variation distance:

$$
|b(x, \mu)-b(x, \nu)| \leqslant \int_{\mathbb{R}}\langle k\rangle^{-\eta}|\lambda(k, \mu)-\lambda(k, \nu)| \mathrm{d} k \leqslant \int_{\mathbb{R}}\langle k\rangle^{-\eta} \Lambda(k) \mathrm{d} k \mathrm{~d}_{\mathrm{TV}}(\mu, \nu)^{\delta} .
$$

Since $\eta \geqslant 0$ and $\Lambda \in L_{1}(\mathbb{R}), \int_{\mathbb{R}}\langle k\rangle^{-\eta} \Lambda(k) \mathrm{d} k$ is finite.

In order to apply our strategy, we need to assume the following minimal regularity assumption on the drift $b$ :

$$
\eta>\frac{3}{2}(1-\delta)
$$

It describes a continuum of admissible drift functions $b$ between the following two extremal classes:

- if $\delta=0$ : the drift is only bounded in the measure variable. In that case, $\eta$ has to satisfy $\eta>\frac{3}{2}$ : this coincide exactly with the assumptions of Theorem 17, where we assumed that for each $\mu \in \mathcal{P}_{2}(\mathbb{R}), b(\cdot, \mu)$ belongs to $H^{\eta}(\mathbb{R})$ with a uniform $H^{\eta}$-norm for some $\eta \geqslant \alpha>\frac{3}{2}$.

- if $\delta=1$ : the drift is Lipschitz-continuous in total variation distance with respect to the measure argument. Jourdain [Jou97], Mishura-Veretennikov [MV], Lacker [Lac18], Chaudru de Raynal-Frikha [CdRF], Röckner-Zhang [RZ] among others have proved results under this assumption if $b$ is only measurable and bounded in the space variable. Our result applies if $b$ belongs to $H^{\eta}(\mathbb{R})$ for some $\eta>0$ and if the Fourier transform of $b$ belongs to $L_{1}(\mathbb{R})$; it is a subset of the space $\mathcal{C}_{0}(\mathbb{R})$ of continuous functions vanishing at infinity.

\section{II.2 Definition of the notion of solution}

Let us consider a new model, with the purpose to make a link between the results obtained in this paper and recent regularization by noise results for McKean-Vlasov equations obtained among others by [Jou97, MV, Lac18, CdRF, RZ]. There are some important changes with respect to the model (13) previously studied in this work. The main modification consists in adding a Brownian motion $\beta$, independent of $w$, in order to take benefit from some additional regularizing effect. In short, the role of $\beta$ in the model below is to smooth out the (finite dimensional) space variable in the drift coefficient. Obviously, this comes in contrast with the role of the Brownian sheet $w$, the action of which is to mollify the velocity field in the measure argument, as made clear by Theorem 17. Of course, we know from the standard diffusive case (i.e. $w \equiv 0$ and $b(x, \mu) \equiv b(x))$ that, in order to fully benefit from the action of $\beta$ onto the space variable, we should average out over all the possible realizations of $\beta$ (for instance, we may consider the semi-group generated by the diffusion process). In the present context, this prompts us to disentangle the roles of the two noises $\beta$ and $w$ in the mean-field interaction. Similarly to the standard McKean-Vlasov model, we shall compute the law of the particle (i.e. the mean-field component) with respect to the noise carrying $\beta$ and the initial condition, but, similarly to the model addressed in the previous section, we shall freeze the realization of $w$. According to the terminology that has been used in the literature (see in particular the mean-field game literature [GLL11, CD18], see also the earlier references [Vai88, DV95, KX99, KX04, CF16]), $\beta$ will be regarded as an idiosyncratic noise acting independently on each particle and $w$ as a common (or systemic) noise. To sum-up, in the previous sections, we defined $\mu_{t}$ as $\mu_{t}=\operatorname{Leb}_{[0,1]} \circ\left(z_{t}\right)^{-1}$, the space $[0,1]$ therein carrying the initial condition in the form $z_{0}(u)=g(u)$ for $u \in[0,1]$. Implicitly, this allowed us to identify $\mu_{t}$ with the conditional law of $z_{t}$ given $(w(k, s))_{k \in \mathbb{R}, s \leqslant t}$. Now, $\mu_{t}$ will be understood as the law of the particle over the randomness carrying both $\beta$ and the initial condition. This idea is made more precise in Remark 25. 
There are two other modifications of the model introduced in this section. In the Girsanov's arguments that we will use in the following proofs, we will not be able to preserve the monotonicity of the solution with respect to the variable $u$ as in the first part. So we decide to use the same framework as usual in the literature on McKean-Vlasov SDEs, namely we take as initial condition a random variable $\xi$ of prescribed law, independent from $\beta$ and $w$. Furthermore, we decide to consider the easiest possible assumption on the mass, namely that it is constant equal to one.

Let $\eta>0$ and $\delta \in[0,1]$ be such that $\eta>\frac{3}{2}(1-\delta)$. Let $b: \mathbb{R} \times \mathcal{P}_{2}(\mathbb{R}) \rightarrow \mathbb{R}$ be of class $\left(H^{\eta}, \mathcal{C}^{\delta}\right)$. Let $f: \mathbb{R} \rightarrow \mathbb{R}$ be a function of order $\alpha$, such that

$$
\frac{3}{2}<\alpha \leqslant \frac{\eta}{1-\delta}
$$

(if $\delta=1$, we just require that $\alpha>\frac{3}{2}$ ). The condition $\eta>\frac{3}{2}(1-\delta)$ insures that this choice of $\alpha$ is possible. Let $\mu_{0}$ be any given initial condition in $\mathcal{P}_{2}(\mathbb{R})$. Let us consider the following SDE:

$$
\left\{\begin{aligned}
\mathrm{d} z_{t} & =\int_{\mathbb{R}} f(k) \Re\left(e^{-i k z_{t}} \mathrm{~d} w(k, t)\right)+\mathrm{d} \beta_{t}+b\left(z_{t}, \mu_{t}\right) \mathrm{d} t \\
\mu_{t} & =\mathcal{L}^{\mathbb{P}}\left(z_{t} \mid \mathcal{G}_{t}^{\mu, W}\right) \text { a.s. } \\
z_{0} & =\xi, \quad \mathcal{L}^{\mathbb{P}}(\xi)=\mu_{0}
\end{aligned}\right.
$$

where the filtration $\left(\mathcal{G}_{t}^{\mu, W}\right)_{t \in[0, T]}$ is defined by $\mathcal{G}_{t}^{\mu, W}:=\sigma\left\{w(\cdot, s), \mu_{s} ; s \leqslant t\right\}$ and where $(\mu, w)$ is independent of $(\beta, \xi)$. Note: In that equation and in all this section, $\mathcal{L}^{\mathbb{P}}(X)$ denotes the law of the random variable $X$ under the probability measure $\mathbb{P}$, that is the distribution $\mathbb{P} \circ X^{-1}$.

Let us define the notion of weak solution to (35):

Definition 24. An element $\boldsymbol{\Omega}=\left(\Omega, \mathcal{G},\left(\mathcal{G}_{t}\right)_{t \in[0, T]}, \mathbb{P}, z, w, \beta, \xi\right)$ is said to be a weak solution to equation (35) if

- $\left(\Omega, \mathcal{G},\left(\mathcal{G}_{t}\right)_{t \in[0, T]}, \mathbb{P}\right)$ is a filtered probability space satisfying usual conditions,

- $(w, \beta, \xi)$ are independent random variables on $(\Omega, \mathcal{G})$, where

$\triangleright w:=\left(w^{\Re}, w^{\Im}\right)$, with $\left(w^{\Re}(k, t)\right)_{k \in \mathbb{R}, t \in[0, T]}$ and $\left(w^{\Im}(k, t)\right)_{k \in \mathbb{R}, t \in[0, T]}$ two independent

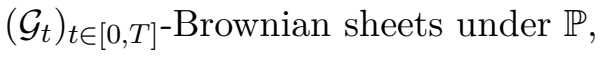

$\triangleright\left(\beta_{t}\right)_{t \in[0, T]}$ is a standard $\left(\mathcal{G}_{t}\right)_{t \in[0, T]}$-Brownian motion under $\mathbb{P}$,

$\triangleright$ for any $t \in[0, T]$, the $\sigma$-field $\sigma\left\{w^{\Re}\left(k, t^{\prime}\right)-w^{\Re}(k, t), w^{\Im}\left(k, t^{\prime}\right)-w^{\Im}(k, t), \beta_{t^{\prime}}-\beta_{t}\right.$; $\left.k \in \mathbb{R}, t^{\prime} \in[t, T]\right\}$ is independent of $\mathcal{G}_{t}$ under $\mathbb{P}$,

$\triangleright \xi$ has distribution $\mu_{0}$ under $\mathbb{P}$;

- $\left(z_{t}\right)_{t \in[0, T]}$ is a continuous $\left(\mathcal{G}_{t}\right)_{t \in[0, T]}$-adapted process satisfying $\mathbb{P}$-almost surely, for every $t \in[0, T]$,

$$
z_{t}=\xi+\int_{0}^{t} \int_{\mathbb{R}} f(k) \Re\left(e^{-i k z_{s}} \mathrm{~d} w(k, s)\right)+\beta_{t}+\int_{0}^{t} b\left(z_{s}, \mu_{s}\right) \mathrm{d} s .
$$

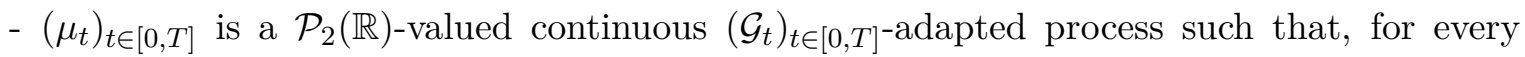
$t \in[0, T], \mathbb{P}$-almost surely, $\mu_{t}=\mathcal{L}^{\mathbb{P}}\left(z_{t} \mid \mathcal{G}_{t}^{\mu, W}\right)$, where $\mathcal{G}_{t}^{\mu, W}:=\sigma\left\{w(k, s), \mu_{s} ; k \in \mathbb{R}, s \leqslant t\right\}$,

- compatibility condition: $(\mu, w)$ is independent of $(\beta, \xi)$ under $\mathbb{P}$ (and thus $(\mu, w), \beta$ and $\xi$ are independent) and, more generally, for every $t \in[0, T]$, the processes $(\xi, w, \mu)$ and $\beta$ are conditionally independent given $\mathcal{G}_{t}$. 
Remark 25. The last two conditions are certainly the most difficult ones to understand. In fact, both are dictated by the fact that we are looking for weak solutions only: a priori, nothing is said on the measurability of $z$ and $\mu$ with respect to the inputs $\xi, w$ and $\beta$. In particular, at this stage, $\mu$ may not be measurable with respect to $w$ (which comes in contrast with the intuitive explanations we gave in introduction of the section). This is the rationale for defining the McKean-Vlasov constraint in terms of the conditional law of $z_{t}$ given the $\sigma$-field generated (up to time $t$ ) not only by $w$ but also by $\mu$ itself. Similarly, the compatibility condition has been widely used (in a slightly stronger manner) in the analysis of weak solutions to stochastic equations, see for instance [Kur07, Kur14]. In short, it says that the observation of $z$ does not corrupt the independence property of $(\xi, \mu, w)$ and $\beta$. Quite obviously, see for instance [CD18, Remark I.11], compatibility is automatically satisfied if $\mu$ is adapted with respect to the completion of $\mathcal{G}^{W}$, in which case the solution should be called semi-strong.

We will prove weak well-posedness for the SDE (35) in three steps: $i$ ) when the drift $b$ is equal to zero; $i i)$ when the drift $b$ is bounded and Lipschitz-continuous in total variation distance with respect to the measure variable; essentially, we will adapt to our case the proof given by Lacker [Lac18], where we will make use of the averaging over the noise $\beta$; iii) in the general case, when the drift $b$ belongs to the class $\left(H^{\eta}, \mathcal{C}^{\delta}\right)$ : we will use here the same arguments as in the first part, using the infinite-dimensional Brownian sheet $w$ to mollify $b$ in the measure argument.

Let us first consider the case where the drift is zero:

$$
\left\{\begin{aligned}
\mathrm{d} z_{t} & =\int_{\mathbb{R}} f(k) \Re\left(e^{-i k z_{t}} \mathrm{~d} w(k, t)\right)+\mathrm{d} \beta_{t}, \\
z_{0} & =\xi, \quad \mathcal{L}^{\mathbb{P}}(\xi)=\mu_{0} .
\end{aligned}\right.
$$

In this case, well-posedness holds even in a strong sense.

Proposition 26. Let $f: \mathbb{R} \rightarrow \mathbb{R}$ be a function of order $\alpha>\frac{3}{2}$. Then there is a unique strong solution to equation (36). Moreover, if $\boldsymbol{\Omega}^{\mathbf{1}}$ and $\boldsymbol{\Omega}^{\mathbf{2}}$ are two solutions to (36), then $\mathcal{L}^{\mathbb{P}^{1}}\left(z^{1}, w^{1}, \beta^{1}\right)=\mathcal{L}^{\mathbb{P}^{2}}\left(z^{2}, w^{2}, \beta^{2}\right)$.

Proof. Strong well-posedness can be proved by a classical fixed-point argument, as in the proof of Proposition 3 for example (but the proof is now easier since the mass is equal to 1 everywhere). The additional noise $\beta$ does not change anything to this proof. Moreover, the assumption $\alpha>\frac{3}{2}$ insures that the assumption of square integrability of $k \mapsto\langle k\rangle f(k)$ is satisfied (see Proposition 3); in other words, it insures that the diffusive coefficient in front of the noise $w$ is Lipschitz-continuous.

Furthermore, by Yamada-Watanabe Theorem, the law of $(z, w, \beta)$ solution to (36) is uniquely determined. That result is stated and proved in [KS91, Prop 5.3.20, p.309] in a finite-dimensional case, but the proof is the same for an infinite dimensional noise. Moreover, a corollary to Yamada-Watanabe Theorem [KS91, Cor 5.3.23, p.310] states the following result: if $\boldsymbol{\Omega}=$ $\left(\Omega, \mathcal{G},\left(\mathcal{G}_{t}\right)_{t \in[0, T]}, \mathbb{P}, z, w, \beta, \xi\right)$ is a solution to $(36)$, then $\mathbb{P}$-almost surely, for every $t \in[0, T]$,

$$
z_{t}=\mathcal{Z}_{t}(\xi, w, \beta)
$$

where $\mathcal{Z}$ is a function defined on the canonical space

$$
\begin{aligned}
\mathcal{Z}: \mathbb{R} \times \mathcal{C}\left(\mathbb{R} \times[0, T], \mathbb{R}^{2}\right) \times \mathcal{C}([0, T], \mathbb{R}) & \rightarrow \mathcal{C}([0, T], \mathbb{R}) \\
\left(x, \omega^{W}, \omega^{\beta}\right) & \mapsto \mathcal{Z}\left(x, \omega^{W}, \omega^{\beta}\right)
\end{aligned}
$$

which is progressively measurable with respect to the canonical filtration on $\mathcal{C}\left(\mathbb{R} \times[0, T], \mathbb{R}^{2}\right) \times$ $\mathcal{C}([0, T], \mathbb{R})$. Remark that $\mathcal{C}([0, T], \mathbb{R})$ represents here the canonical space on which we define the Wiener measure of a standard Wiener process on $[0, T]$, and $\mathcal{C}\left(\mathbb{R} \times[0, T], \mathbb{R}^{2}\right)$ represents the Wiener space associated to the measure of a $\mathbb{R}^{2}$-valued Brownian sheet $\left(w^{\Re}, w^{\Im}\right)$ on $\mathbb{R} \times[0, T]$. 


\section{II.3 Resolution of the SDE when the drift is Lipschitz continuous}

Let us assume that $\tilde{b}: \mathbb{R} \times \mathcal{P}_{2}(\mathbb{R}) \rightarrow \mathbb{R}$ is uniformly bounded and uniformly Lipschitz-continuous in total variation distance in the measure variable. We consider the following SDE with the drift $\widetilde{b}$ :

$$
\left\{\begin{aligned}
\mathrm{d} z_{t} & =\int_{\mathbb{R}} f(k) \Re\left(e^{-i k z_{t}} \mathrm{~d} w(k, t)\right)+\mathrm{d} \beta_{t}+\widetilde{b}\left(z_{t}, \mu_{t}\right) \mathrm{d} t, \\
\mu_{t} & =\mathcal{L}^{\mathbb{P}}\left(z_{t} \mid \mathcal{G}_{t}^{\mu, W}\right), \\
z_{0} & =\xi, \quad \mathcal{L}^{\mathbb{P}}(\xi)=\mu_{0},
\end{aligned}\right.
$$

with the same assumptions and the same interpretation as in Definition 24. Let us prove existence and uniqueness of a weak solution.

Proposition 27. Let $f: \mathbb{R} \rightarrow \mathbb{R}$ be a function of order $\alpha>\frac{3}{2}$. Let $\widetilde{b}: \mathbb{R} \times \mathcal{P}_{2}(\mathbb{R}) \rightarrow \mathbb{R}$ be $a$ function such that there exists $C>0$ satisfying for every $x \in \mathbb{R}$ and for every $\mu, \nu \in \mathcal{P}_{2}(\mathbb{R})$

$$
\begin{aligned}
& \text { - }|\widetilde{b}(x, \mu)| \leqslant C ; \\
& \text { - }|\widetilde{b}(x, \mu)-\widetilde{b}(x, \nu)| \leqslant C \mathrm{~d}_{\mathrm{TV}}(\mu, \nu) .
\end{aligned}
$$

Then there exists a weak solution to (38).

Proposition 28. Under the same assumptions as Proposition 27 , if $\boldsymbol{\Omega}^{\mathbf{1}}$ and $\boldsymbol{\Omega}^{\mathbf{2}}$ are two weak solutions to $(38)$, then $\mathcal{L}^{\mathbb{P}^{1}}\left(z^{1}, w^{1}\right)=\mathcal{L}^{\mathbb{P}^{2}}\left(z^{2}, w^{2}\right)$. In particular, uniqueness in law holds for the SDE (38). Moreover, for any weak solution $\boldsymbol{\Omega},\left(\mu_{t}\right)_{t \in[0, T]}$ is adapted to the completion of $\left(\mathcal{G}_{t}^{W}=\sigma\{w(\cdot, s) ; s \leqslant t\}\right)_{t \in[0, T]}$.

Note that the statement of Proposition 28 shows that the weak solution of (38) is adapted to the filtration generated by the noise $w$.

Remark 29. The question of the filtration under which the measure-valued process $\left(\mu_{t}\right)_{t \in[0, T]}$ is adapted is important here. Actually, we will see in the proof of existence that the weak solution that we will construct is automatically adapted with respect to the filtration generated by $w$. Nevertheless, we want to give a more general statement for uniqueness, i.e. we want to be able to compare two weak solutions where $\left(\mu_{t}\right)_{t \in[0, T]}$ is adapted with respect to a filtration generated by $w$ and possibly another source of randomness, provided $(\mu, W)$ remains independent of $(\beta, \xi)$. This will be useful in the proof of Theorem 32, which states well-posedness for the SDE with $\left(H^{\eta}, \mathcal{C}^{\delta}\right)$-drift $b$, since for this general case, our proof based on Girsanov's Theorem does not imply that $\left(\mu_{t}\right)_{t \in[0, T]}$ is adapted with respect to the filtration generated by $w$ (see Remark 34).

The assumptions on $\widetilde{b}$ are the same as in [Lac18]. We will essentially apply the same proof, which we will recall hereafter.

\section{II.3.1 Existence of a weak solution to the intermediate SDE}

Let us prove in this paragraph Proposition 27. We begin by constructing a weak solution on the canonical space.

Proof (Proposition 27). Let us consider the filtered canonical probability space, denoted by $\left(\Omega^{W}, \mathcal{G}^{W},\left(\mathcal{G}_{t}^{W}\right)_{t \in[0, T]}, \mathbb{P}^{W}\right)$, where $\Omega^{W}:=\mathcal{C}\left(\mathbb{R} \times[0, T], \mathbb{R}^{2}\right), \mathcal{G}^{W}$ is the Borel $\sigma$-algebra on $\Omega^{W},\left(\mathcal{G}_{t}^{W}\right)_{t \in[0, T]}$ is the canonical filtration on $\left(\Omega^{W}, \mathcal{G}^{W}\right)$ and $\mathbb{P}^{W}$ is the probability measure on $\left(\Omega^{W}, \mathcal{G}^{W}\right)$ such that the distribution of the random variable $w^{W} \mapsto w^{W}$ is the law of two independent (real-valued) Brownian sheets on $\mathbb{R} \times[0, T]$. 
Let $\left(\Omega^{\beta, \xi}, \mathcal{G}^{\beta, \xi},\left(\mathcal{G}_{t}^{\beta, \xi}\right)_{t \in[0, T]}, \mathbb{P}^{\beta, \xi}\right)$ be another filtered probability space on which we define two independent random variables $\xi$ and $\left(\beta_{t}\right)_{t \in[0, T]}$ such that $\left(\beta_{t}\right)_{t \in[0, T]}$ is a $\left(\mathcal{G}_{t}^{\beta, \xi}\right)_{t \in[0, T]}$-adapted Brownian motion and such that the law of $\xi$ is $\mu_{0}$.

Let $\left(\Omega, \mathcal{G},\left(\mathcal{G}_{t}\right)_{t \in[0, T]}, \mathbb{P}\right)$ be the product space: $\Omega=\Omega^{W} \times \Omega^{\beta, \xi}, \mathcal{G}=\mathcal{G}^{W} \otimes \mathcal{G}^{W, \beta}, \mathcal{G}_{t}=$ $\sigma\left(\mathcal{G}_{t}^{W}, \mathcal{G}_{t}^{\beta, \xi}\right)$ and $\mathbb{P}=\mathbb{P}^{W} \otimes \mathbb{P}^{\beta, \xi}$. In particular, $w$ is independent of $(\beta, \xi)$ under $\mathbb{P}$. Up to adding negligible subsets, we assume that the filtration $\left(\mathcal{G}_{t}\right)_{t \in[0, T]}$ is complete. Let $\left(z_{t}\right)_{t \in[0, T]}$ be the unique solution on $\left(\Omega, \mathcal{G},\left(\mathcal{G}_{t}\right)_{t \in[0, T]}, \mathbb{P}\right)$ of the $\mathrm{SDE}$ :

$$
\left\{\begin{aligned}
\mathrm{d} z_{t} & =\int_{\mathbb{R}} f(k) \Re\left(e^{-i k z_{t}} \mathrm{~d} w(k, t)\right)+\mathrm{d} \beta_{t}, \\
z_{0} & =\xi, \quad \mathcal{L}^{\mathbb{P}}(\xi)=\mu_{0} .
\end{aligned}\right.
$$

Existence and uniqueness of a strong solution to (39) is given by Proposition 26. Furthermore, by Yamada-Watanabe Theorem, there is a $\left(\mathcal{G}_{t}\right)_{t \in[0, T]}$-progressively measurable map $\mathcal{Z}_{t}$ as defined in (37) such that $\mathbb{P}$-almost surely, $z_{t}=\mathcal{Z}_{t}(\xi, w, \beta)$.

Let us denote by $\mathcal{C}$ the space $\mathcal{C}([0, T], \mathbb{R})$ and by $\mathcal{P}(\mathcal{C})$ the space of probability measures on $\mathcal{C}$. For each time $t \in[0, T]$, let us denote by $\pi_{t}: \mu^{T} \in \mathcal{P}(\mathcal{C}) \mapsto \mu^{t} \in \mathcal{P}(\mathcal{C})$ the map associating to $\mu^{T}$ the push-forward measure of $\mu^{T}$ by the map $x \in \mathcal{C} \mapsto x \cdot \wedge t \in \mathcal{C}$. Let $(\mathcal{X}, d)$ be the complete metric space of functions

$$
\begin{aligned}
\mu: \Omega^{W}=\mathcal{C}\left(\mathbb{R} \times[0, T], \mathbb{R}^{2}\right) & \rightarrow \mathcal{P}(\mathcal{C}) \\
w & \mapsto \mu^{T}(w),
\end{aligned}
$$

such that, for each $t \in[0, T],\left(\mu^{t}=\pi^{t}\left(\mu^{T}\right)\right)_{t \in[0, T]}$ is $\left(\mathcal{G}_{t}^{W}\right)_{t \in[0, T]}$-progressively measurable. The distance $d$ is defined by $d(\mu, \nu):=\mathbb{E}^{W}\left[\mathrm{~d}_{\mathrm{TV}}\left(\mu^{T}, \nu^{T}\right)^{2}\right]^{1 / 2}$, where $\mathrm{d}_{\mathrm{TV}}$ is here understood as the total variation distance on $\mathcal{P}(\mathcal{C})$ (while we defined it before on $\mathcal{P}(\mathbb{R})$ ). Furthermore, for $\mu \in \mathcal{X}$ and for $t \in[0, T]$, we call $\mu_{t}$ the image of $\mu$ by the mapping $x \in \mathcal{C} \mapsto x_{t} \in \mathbb{R}$.

Let $\nu \in \mathcal{X}$. Recall that $\widetilde{b}: \mathbb{R} \times \mathcal{P}_{2}(\mathbb{R}) \rightarrow \mathbb{R}$ is uniformly bounded. Therefore

$$
\mathcal{E}_{t}^{\nu}:=\exp \left(\int_{0}^{t} \widetilde{b}\left(z_{s}, \nu_{s}\right) \mathrm{d} \beta_{s}-\frac{1}{2} \int_{0}^{t}\left|\widetilde{b}\left(z_{s}, \nu_{s}\right)\right|^{2} \mathrm{~d} s\right)
$$

is a $\left(\mathcal{G}_{t}\right)_{t \in[0, T]}$-martingale. Let $\mathbb{P}^{\nu}$ be the probability measure on $(\Omega, \mathcal{G})$ absolutely continuous with respect to $\mathbb{P}=\mathbb{P}^{W} \otimes \mathbb{P}^{\beta, \xi}$, with density:

$$
\frac{\mathrm{d} \mathbb{P}^{\nu}}{\mathrm{dP}}=\mathcal{E}_{T}^{\nu}
$$

For every $w \in \Omega^{W}$, let us denote by $\mathbb{P}^{\nu, \beta, \xi}(w)$ the probability measure on $\left(\Omega^{\beta, \xi}, \mathcal{G}^{\beta, \xi}\right)$ with the following density with respect to $\mathbb{P}^{\beta, \xi}$ :

$$
\frac{\mathrm{d} \mathbb{P}^{\nu, \beta, \xi}(w)}{\mathrm{d} \mathbb{P}^{\beta, \xi}}=\mathcal{E}_{T}^{\nu}(w)
$$

Equivalently, $\mathbb{P}^{\nu, \beta, \xi}: \Omega^{W} \times \mathcal{G}^{\beta, \xi} \rightarrow \mathbb{R}_{+}$is also defined as the conditional probability satisfying for every $\left(A^{W}, A^{\beta, \xi}\right) \in \mathcal{G}^{W} \times \mathcal{G}^{\beta, \xi}$

$$
\mathbb{P}^{\nu}\left(A^{W} \times A^{\beta, \xi}\right)=\int_{A^{W}} \mathbb{P}^{\nu, \beta, \xi}\left(w, A^{\beta, \xi}\right) \mathrm{d} \mathbb{P}^{W}(w) .
$$

Let us define $\mathrm{d} \widetilde{\beta}_{t}^{\nu}:=\mathrm{d} \beta_{t}-\widetilde{b}\left(z_{t}, \nu_{t}\right) \mathrm{d} t$. By Girsanov's Theorem, $\left(\widetilde{\beta}_{t}^{\nu}\right)_{t \in[0, T]}$ is a Brownian motion under the measure $\mathbb{P}^{\nu},\left(\widetilde{\beta}^{\nu}, \xi, w\right)$ are independent under $\mathbb{P}^{\nu}$ and, for any $t \in[0, T]$, the $\sigma$-field 
$\sigma\left\{w\left(k, t^{\prime}\right)-w(k, t), \widetilde{\beta}_{t^{\prime}}^{\nu}-\widetilde{\beta}_{t}^{\nu} ; k \in \mathbb{R}, t^{\prime} \in[t, T]\right\}$ is independent of $\mathcal{G}_{t}$ under $\mathbb{P}^{\nu}$. Moreover the process $\left(z_{t}\right)_{t \in[0, T]}$ satisfies:

$$
\mathrm{d} z_{t}=\int_{\mathbb{R}} f(k) \Re\left(e^{-i k z_{t}} \mathrm{~d} w(k, t)\right)+\mathrm{d} \widetilde{\beta}_{t}^{\nu}+\widetilde{b}\left(z_{t}, \nu_{t}\right) \mathrm{d} t .
$$

If $\nu$ satisfies for every $t \in[0, T], \mathbb{P}^{W}$-almost surely,

$$
\nu_{t}=\mathcal{L}^{\mathbb{P}^{\nu}}\left(z_{t} \mid \mathcal{G}_{t}^{W}\right)
$$

then it also satisfies for every $t \in[0, T], \mathbb{P}^{W}$-almost surely, $\nu_{t}=\mathcal{L}^{\mathbb{P}^{\nu}}\left(z_{t} \mid \mathcal{G}_{t}^{\nu, W}\right)$, where $\mathcal{G}_{t}^{\nu, W}=$ $\sigma\left\{w(\cdot, s), \nu_{s} ; s \leqslant t\right\}$. Furthermore, $(\nu, w)$ is adapted to the completion of $\mathcal{G}^{W}$; hence under $\mathbb{P}^{\nu},(\nu, w)$ is independent of $\left(\tilde{\beta}^{\nu}, \xi\right)$, and by Remark 25 the compatibility condition is automatically satisfied. Thus if (40) is satisfied for any $t \in[0, T] \mathbb{P}^{W}$-almost surely, then $\left(\Omega, \mathcal{G},\left(\mathcal{G}_{t}\right)_{t \in[0, T]}, \mathbb{P}^{\nu}, z, w, \widetilde{\beta}^{\nu}, \xi\right)$ is a weak solution to (38). Equivalently, it is solution if for $\mathbb{P}^{W}$-almost every $w \in \Omega^{W}$, for every $t \in[0, T], \nu_{t}(w)=\mathcal{L}^{\mathbb{P}^{\nu, \beta, \xi}(w)}\left(\mathcal{Z}_{t}(\cdot, w, \cdot)\right)$ (the latter obviously implying (40) and the converse following from the fact that, in (40), $\mathcal{G}_{t}^{W}$ can be replaced by $\mathcal{G}_{T}^{W}$, which implies not only that, for any $t \in[0, T]$, for $\mathbb{P}^{W}$-almost every $w \in \Omega^{W}$, $\nu_{t}(w)=\mathcal{L}^{\mathbb{P}^{\nu, \beta, \xi}(w)}\left(\mathcal{Z}_{t}(\cdot, w, \cdot)\right)$ but also that the quantifiers for all $t$ and for $\mathbb{P}^{W}$-almost every can be exchanged by a standard continuity argument). Notice in particular, that by Fubini's Theorem, $w \mapsto \mathcal{L}^{\mathbb{P}^{\nu, \beta, \xi}(w)}\left(\mathcal{Z}_{t}(\cdot, w, \cdot)\right)$ is $\mathcal{G}_{t}^{W}$-measurable.

Let us prove that there is a process $\nu \in \mathcal{X}$ satisfying (40). For every $\nu \in \mathcal{X}$, let us define $\phi(\nu)_{t}:=w \mapsto \mathcal{L}^{\mathbb{P}^{\nu, \beta, \xi}(w)}\left(\mathcal{Z}_{t}(\cdot, w, \cdot)\right)$. By construction, $\phi(\nu)$ also belongs to $\mathcal{X}$. For every $\mu^{T} \in \mathcal{X}$ and for every $t \in[0, T]$, let us denote by $\mu^{t} \in \mathcal{P}(\mathcal{C})$ the push-forward measure of $\mu^{T}$ through the map $x \in \mathcal{C} \mapsto x_{. \wedge t} \in \mathcal{C}$. In particular, for every $t \in[0, T], \phi(\nu)^{t}=\mathcal{L}^{\mathbb{P}^{\nu, \beta, \xi}(w)}\left(\mathcal{Z}_{\cdot \wedge t}(\cdot, w, \cdot)\right)$. For $\mu, \nu \in \mathcal{P}_{2}(\mathcal{C})$, let us denote by $H(\mu \mid \nu)$ the relative entropy

$$
H(\mu \mid \nu)=\int_{\mathcal{C}} \ln \frac{\mathrm{d} \mu}{\mathrm{d} \nu} \mathrm{d} \mu \quad \text { if } \mu \ll \nu, \quad H(\mu \mid \nu)=+\infty \quad \text { otherwise. }
$$

Here, we apply the same strategy of proof as in [Lac18, Thm 2.4]. Let us state the following lemma, which is shown at the end of the current proof.

Lemma 30. For every $\mu, \nu \in \mathcal{X}$ and for every $t \in[0, T]$,

$$
H\left(\phi(\mu)^{t} \mid \phi(\nu)^{t}\right)=\frac{1}{2} \mathbb{E}^{\mathbb{P}^{\mu}}\left[\int_{0}^{t}\left|\widetilde{b}\left(z_{s}, \nu_{s}\right)-\widetilde{b}\left(z_{s}, \mu_{s}\right)\right|^{2} \mathrm{~d} s \mid \mathcal{G}_{t}^{W}\right] .
$$

By Lipschitz-continuity of $\widetilde{b}$, there is $C>0$ such that

$$
\begin{aligned}
H\left(\phi(\mu)^{t} \mid \phi(\nu)^{t}\right) \leqslant C \int_{0}^{t} \mathbb{E}^{\mathbb{P}^{\mu}}\left[\mathrm{d}_{\mathrm{TV}}\left(\mu_{s}, \nu_{s}\right)^{2} \mid \mathcal{G}_{t}^{W}\right] \mathrm{d} s & =C \int_{0}^{t} \mathrm{~d}_{\mathrm{TV}}\left(\mu_{s}, \nu_{s}\right)^{2} \mathrm{~d} s \\
& \leqslant C \int_{0}^{t} \mathrm{~d}_{\mathrm{TV}}\left(\mu^{s}, \nu^{s}\right)^{2} \mathrm{~d} s
\end{aligned}
$$

By Pinsker's inequality, $\mathrm{d}_{\mathrm{TV}}\left(\phi(\mu)^{t}, \phi(\nu)^{t}\right)^{2} \leqslant 2 H\left(\phi(\mu)^{t} \mid \phi(\nu)^{t}\right)$. Therefore, there is $C$ such that for every $t \in[0, T]$,

$$
\mathbb{E}^{W}\left[\mathrm{~d}_{\mathrm{TV}}\left(\phi(\mu)^{t}, \phi(\nu)^{t}\right)^{2}\right] \leqslant C \int_{0}^{t} \mathbb{E}^{W}\left[\mathrm{~d}_{\mathrm{TV}}\left(\mu^{s}, \nu^{s}\right)^{2}\right] \mathrm{d} s .
$$

For every $n \in \mathbb{N} \backslash\{0\}$, let us write $\phi^{\circ n}$ for $\underbrace{\phi \circ \cdots \circ \phi}_{n \text { times }}$. It follows from a simple recursion and from (41) that for every $t \in[0, T]$ and $n \in \mathbb{N}$

$$
\mathbb{E}^{W}\left[\mathrm{~d}_{\mathrm{TV}}\left(\phi^{\circ n}(\mu)^{t}, \phi^{\circ n}(\nu)^{t}\right)^{2}\right] \leqslant \frac{C^{n} t^{n}}{n !} \mathbb{E}^{W}\left[\mathrm{~d}_{\mathrm{TV}}\left(\mu^{t}, \nu^{t}\right)^{2}\right] .
$$


Recall that the distance $d$ on $\mathcal{X}$ is defined by $d(\mu, \nu)=\mathbb{E}^{W}\left[\mathrm{~d}_{\mathrm{TV}}\left(\mu^{T}, \nu^{T}\right)^{2}\right]^{1 / 2}$. Thus for every $n \geqslant 1$ and for every $\mu, \nu \in \mathcal{X}$,

$$
d\left(\phi^{\circ n}(\mu), \phi^{\circ n}(\nu)\right)^{2} \leqslant \frac{C^{n} T^{n}}{n !} d(\mu, \nu)^{2} .
$$

Therefore, for $n$ large enough so that $\frac{C^{n} T^{n}}{n !}<1, \phi^{\text {on }}$ is a contraction. Therefore, by Picard's fixed-point Theorem, there is a unique solution, called $\mu^{\phi} \in \mathcal{X}$, of $\mu^{\phi}=\phi\left(\mu^{\phi}\right)$. In particular, there exists a weak solution to equation (38). This completes the proof of Proposition 27.

Proof (Lemma 30). Let us first compute for every $t \in[0, T]$,

$$
H\left(\phi(\mu)^{t} \mid \phi(\nu)^{t}\right)=\int_{\mathcal{C}} \ln \frac{\mathrm{d} \phi(\mu)^{t}}{\mathrm{~d} \phi(\nu)^{t}} \mathrm{~d} \phi(\mu)^{t}=\mathbb{E}^{\mathbb{P}^{\mu}}\left[\ln \frac{\mathrm{d} \phi(\mu)^{t}}{\mathrm{~d} \phi(\nu)^{t}}(z \cdot \wedge t) \mid \mathcal{G}_{t}^{W}\right] .
$$

Let us prove that

$$
\frac{\mathrm{d} \phi(\mu)^{t}}{\mathrm{~d} \phi(\nu)^{t}}(z \cdot \wedge t)=\mathbb{E}^{\mathbb{P}^{\nu}}\left[\frac{\mathrm{d} \mathbb{P}^{\mu}}{\mathrm{d} \mathbb{P}^{\nu}} \mid \mathcal{G}_{t}^{z, W}\right]
$$

where $\mathcal{G}_{t}^{z, W}=\sigma\left\{z_{s}, w(\cdot, s) ; s \leqslant t\right\}$. Indeed, for every measurable and bounded functions $f: \mathcal{C}([0, T], \mathbb{R}) \rightarrow \mathbb{R}$ and $g: \mathcal{C}\left(\mathbb{R} \times[0, T], \mathbb{R}^{2}\right) \rightarrow \mathbb{R}$ (recall that we denote by $\mathcal{C}$ the space $\mathcal{C}([0, T], \mathbb{R}))$ :

$$
\begin{aligned}
& \mathbb{E}^{\mathbb{P}^{\nu}}\left[\frac{\mathrm{d} \mathbb{P}^{\mu}}{\mathrm{d} \mathbb{P}^{\nu}} f(z \cdot \wedge t) g(w \cdot \wedge t)\right]=\mathbb{E}^{\mathbb{P}^{\mu}}[f(z \cdot \wedge t) g(w \cdot \wedge t)]=\mathbb{E}^{\mathbb{P}^{\mu}}\left[\int_{\mathcal{C}} f(x) \mathrm{d} \phi(\mu)^{t}(x) g(w \cdot \wedge t)\right] \\
& =\mathbb{E}^{\mathbb{P}^{\mu}}\left[\int_{\mathcal{C}} f(x) \frac{\mathrm{d} \phi(\mu)^{t}}{\mathrm{~d} \phi(\nu)^{t}}(x) \mathrm{d} \phi(\nu)^{t}(x) g(w \cdot \wedge t)\right]=\mathbb{E}^{\mathbb{P}^{\nu}}\left[\frac{\mathrm{d} \mathbb{P}^{\mu}}{\mathrm{d} \mathbb{P}^{\nu}} \int_{\mathcal{C}} f(x) \frac{\mathrm{d} \phi(\mu)^{t}}{\mathrm{~d} \phi(\nu)^{t}}(x) \mathrm{d} \phi(\nu)^{t}(x) g(w \cdot \wedge t)\right] \\
& =\mathbb{E}^{\mathbb{P}^{\nu}}\left[\mathbb{E}^{\mathbb{P}^{\nu}}\left[\frac{\mathrm{d} \mathbb{P}^{\mu}}{\mathrm{d} \mathbb{P}^{\nu}} \mid \mathcal{G}_{t}^{W}\right] \int_{\mathcal{C}} f(x) \frac{\mathrm{d} \phi(\mu)^{t}}{\mathrm{~d} \phi(\nu)^{t}}(x) \mathrm{d} \phi(\nu)^{t}(x) g(w \cdot \wedge t)\right] \\
& =\mathbb{E}^{\mathbb{P}^{\nu}}\left[\mathbb{E}^{\mathbb{P}^{\nu}}\left[\frac{\mathrm{d} \mathbb{P}^{\mu}}{\mathrm{d} \mathbb{P}^{\nu}} \mid \mathcal{G}_{t}^{W}\right] f(z \cdot \wedge t) \frac{\mathrm{d} \phi(\mu)^{t}}{\mathrm{~d} \phi(\nu)^{t}}(z \cdot \wedge t) g(w \cdot \wedge t)\right] .
\end{aligned}
$$

Moreover, recalling the relation $\mathrm{d} \beta_{s}=\mathrm{d} \widetilde{\beta}_{s}^{\nu}+\widetilde{b}\left(z_{s}, \nu_{s}\right) \mathrm{d} s$,

$$
\begin{aligned}
& \mathbb{E}^{\mathbb{P}^{\nu}}\left[\frac{\mathrm{d} \mathbb{P}^{\mu}}{\mathrm{d} \mathbb{P}^{\nu}} \mid \mathcal{G}_{t}^{W}\right]=\mathbb{E}^{\mathbb{P}^{\nu}}\left[\mathcal{E}_{T}^{\mu}\left(\mathcal{E}_{T}^{\nu}\right)^{-1} \mid \mathcal{G}_{t}^{W}\right] \\
& =\mathbb{E}^{\mathbb{P}^{\nu}}\left[\exp \left(\int_{0}^{T}\left(\widetilde{b}\left(z_{s}, \mu_{s}\right)-\widetilde{b}\left(z_{s}, \nu_{s}\right)\right) \mathrm{d} \beta_{s}-\frac{1}{2} \int_{0}^{T}\left|\widetilde{b}\left(z_{s}, \mu_{s}\right)\right|^{2} \mathrm{~d} s+\frac{1}{2} \int_{0}^{T}\left|\widetilde{b}\left(z_{s}, \nu_{s}\right)\right|^{2} \mathrm{~d} s\right) \mid \mathcal{G}_{t}^{W}\right] \\
& =\mathbb{E}^{\mathbb{P}^{\nu}}\left[\exp \left(\int_{0}^{T}\left(\widetilde{b}\left(z_{s}, \mu_{s}\right)-\widetilde{b}\left(z_{s}, \nu_{s}\right)\right) \mathrm{d} \widetilde{\beta}_{s}^{\nu}-\frac{1}{2} \int_{0}^{T}\left|\widetilde{b}\left(z_{s}, \mu_{s}\right)-\widetilde{b}\left(z_{s}, \nu_{s}\right)\right|^{2} \mathrm{~d} s\right) \mid \mathcal{G}_{t}^{W}\right] .
\end{aligned}
$$

For every bounded and measurable $g: \mathcal{C}\left(\mathbb{R} \times[0, T], \mathbb{R}^{2}\right) \rightarrow \mathbb{R}$

$$
\begin{aligned}
& \mathbb{E}^{\mathbb{P}^{\nu}}\left[\exp \left(\int_{0}^{T}\left(\widetilde{b}\left(z_{s}, \mu_{s}\right)-\widetilde{b}\left(z_{s}, \nu_{s}\right)\right) \mathrm{d} \widetilde{\beta}_{s}^{\nu}-\frac{1}{2} \int_{0}^{T}\left|\widetilde{b}\left(z_{s}, \mu_{s}\right)-\widetilde{b}\left(z_{s}, \nu_{s}\right)\right|^{2} \mathrm{~d} s\right) g(w \cdot \wedge t)\right] \\
= & \mathbb{E}^{\mathbb{P}^{\nu}}\left[\mathbb{E}^{\mathbb{P}^{\nu, \beta, \xi}(w)}\left[\exp \left(\int_{0}^{T}\left(\widetilde{b}\left(z_{s}, \mu_{s}\right)-\widetilde{b}\left(z_{s}, \nu_{s}\right)\right) \mathrm{d} \widetilde{\beta}_{s}^{\nu}-\frac{1}{2} \int_{0}^{T}\left|\widetilde{b}\left(z_{s}, \mu_{s}\right)-\widetilde{b}\left(z_{s}, \nu_{s}\right)\right|^{2} \mathrm{~d} s\right)\right] g\left(w_{\cdot \wedge t}\right)\right] \\
= & \mathbb{E}^{\mathbb{P}^{\nu}}[g(w \cdot \wedge t)],
\end{aligned}
$$


since under $\mathbb{P}^{\nu}, \widetilde{\beta}^{\nu}$ and $w$ are independent and since the exponential is a $\mathbb{P}^{\nu, \beta, \xi}(w)$-martingale by Novikov's condition (recalling that $\widetilde{b}$ is uniformly bounded). Thus

$$
\mathbb{E}^{\mathbb{P}^{\nu}}\left[\frac{\mathrm{d} \mathbb{P}^{\mu}}{\mathrm{d} \mathbb{P}^{\nu}} \mid \mathcal{G}_{t}^{W}\right]=1
$$

Using equalities (44) and (45), we get equality (43).

Therefore, back to equality (42), we obtain

$$
H\left(\phi(\mu)^{t} \mid \phi(\nu)^{t}\right)=\mathbb{E}^{\mathbb{P}^{\mu}}\left[\ln \mathbb{E}^{\mathbb{P}^{\nu}}\left[\frac{\mathrm{d} \mathbb{P}^{\mu}}{\mathrm{d} \mathbb{P}^{\nu}} \mid \mathcal{G}_{t}^{z, W}\right] \mid \mathcal{G}_{t}^{W}\right] .
$$

Recall that $\left(\Omega, \mathcal{G},\left(\mathcal{G}_{t}\right)_{t \in[0, T]}, \mathbb{P}^{\nu}, z, w, \widetilde{\beta}^{\nu}, \xi\right)$ is a weak solution to (38). Thus $\mathbb{P}^{\nu}$-almost surely, $\widetilde{\beta}^{\nu}$ satisfies for every $t \in[0, T]$ :

$$
\widetilde{\beta}_{t}^{\nu}=z_{t}-z_{0}-\int_{0}^{t} \int_{\mathbb{R}} f(k) \Re\left(e^{-i k z_{s}} \mathrm{~d} w(k, s)\right)-\int_{0}^{t} \widetilde{b}\left(z_{s}, \mu_{s}\right) \mathrm{d} s .
$$

Thus $\left(\widetilde{\beta}_{t}^{\nu}\right)_{t \in[0, T]}$ is $\left(\mathcal{G}_{t}^{z, W}\right)_{t \in[0, T]}$-adapted and we deduce that

$$
\begin{aligned}
H\left(\phi(\mu)^{t} \mid \phi\right. & \left.(\nu)^{t}\right) \\
& =\mathbb{E}^{\mathbb{P}^{\mu}}\left[\ln \exp \left(\int_{0}^{t}\left(\widetilde{b}\left(z_{s}, \mu_{s}\right)-\widetilde{b}\left(z_{s}, \nu_{s}\right)\right) \mathrm{d} \widetilde{\beta}_{s}^{\nu}-\frac{1}{2} \int_{0}^{t}\left|\widetilde{b}\left(z_{s}, \mu_{s}\right)-\widetilde{b}\left(z_{s}, \nu_{s}\right)\right|^{2} \mathrm{~d} s\right) \mid \mathcal{G}_{t}^{W}\right] \\
& =\mathbb{E}^{\mathbb{P}^{\mu}}\left[\int_{0}^{t}\left(\widetilde{b}\left(z_{s}, \mu_{s}\right)-\widetilde{b}\left(z_{s}, \nu_{s}\right)\right) \mathrm{d} \widetilde{\beta}_{s}^{\nu}-\frac{1}{2} \int_{0}^{t}\left|\widetilde{b}\left(z_{s}, \mu_{s}\right)-\widetilde{b}\left(z_{s}, \nu_{s}\right)\right|^{2} \mathrm{~d} s \mid \mathcal{G}_{t}^{W}\right] \\
& =\mathbb{E}^{\mathbb{P}^{\mu}}\left[\int_{0}^{t}\left(\widetilde{b}\left(z_{s}, \mu_{s}\right)-\widetilde{b}\left(z_{s}, \nu_{s}\right)\right) \mathrm{d} \widetilde{\beta}_{s}^{\mu}+\frac{1}{2} \int_{0}^{t}\left|\widetilde{b}\left(z_{s}, \mu_{s}\right)-\widetilde{b}\left(z_{s}, \nu_{s}\right)\right|^{2} \mathrm{~d} s \mid \mathcal{G}_{t}^{W}\right] \\
& =\mathbb{E}^{\mathbb{P}^{\mu}}\left[\frac{1}{2} \int_{0}^{t}\left|\widetilde{b}\left(z_{s}, \mu_{s}\right)-\widetilde{b}\left(z_{s}, \nu_{s}\right)\right|^{2} \mathrm{~d} s \mid \mathcal{G}_{t}^{W}\right],
\end{aligned}
$$

because $\mathrm{d} \widetilde{\beta}_{s}^{\nu}-\mathrm{d} \widetilde{\beta}_{s}^{\mu}=\left(\widetilde{b}\left(z_{s}, \mu_{s}\right)-\widetilde{b}\left(z_{s}, \nu_{s}\right)\right) \mathrm{d} s$. This completes the proof of the Lemma.

\section{II.3.2 Uniqueness in law for the intermediate SDE}

Let us prove in this paragraph Proposition 28.

Proof (Proposition 28). Let $\boldsymbol{\Omega}^{\mathbf{1}}$ and $\boldsymbol{\Omega}^{\mathbf{2}}$ be two weak solutions to (38), often denoted by $\boldsymbol{\Omega}^{\mathbf{n}}$, $n=1,2$. In particular, the process $\left(z_{t}^{n}\right)_{t \in[0, T]}$ satisfies $\mathbb{P}^{n}$-almost surely,

$$
z_{t}^{n}=\xi^{n}+\int_{0}^{t} \int_{\mathbb{R}} f(k) \Re\left(e^{-i k z_{s}^{n}} \mathrm{~d} w^{n}(k, s)\right)+\beta_{t}^{n}+\int_{0}^{t} \widetilde{b}\left(z_{s}^{n}, \mu_{s}^{n}\right) \mathrm{d} s,
$$

where for every $t \in[0, T], \mathbb{P}^{n}$-almost surely $\mu_{t}^{n}=\mathcal{L}^{\mathbb{P}^{n}}\left(z_{t}^{n} \mid \mathcal{G}_{t}^{\mu^{n}, W^{n}}\right)$ and where $\left(\mu^{n}, w^{n}\right)$ is independent of $\left(\beta^{n}, \xi^{n}\right)$.

Let $\mathbb{Q}^{n}$ be the probability measure on $\left(\Omega^{n}, \mathcal{G}^{n}\right)$ with the following density with respect to $\mathbb{P}^{n}$,

$$
\frac{\mathrm{d} \mathbb{Q}^{n}}{\mathrm{~d} \mathbb{P}^{n}}=\exp \left(-\int_{0}^{T} \widetilde{b}\left(z_{s}^{n}, \mu_{s}^{n}\right) \mathrm{d} \beta_{s}^{n}-\frac{1}{2} \int_{0}^{T}\left|\widetilde{b}\left(z_{s}^{n}, \mu_{s}^{n}\right)\right|^{2} \mathrm{~d} s\right) .
$$

Let $\widetilde{\beta}_{t}^{n}=\beta_{t}^{n}+\int_{0}^{t} \widetilde{b}\left(z_{s}^{n}, \mu_{s}^{n}\right) \mathrm{d} s$. By Girsanov's Theorem, $\mathcal{L}^{\mathbb{Q}^{n}}\left(w^{n}, \widetilde{\beta}^{n}, \xi^{n}\right)=\mathcal{L}^{\mathbb{P}^{n}}\left(w^{n}, \beta^{n}, \xi^{n}\right)$ and for any $t \in[0, T]$, the $\sigma$-field $\sigma\left\{w^{n}\left(k, t^{\prime}\right)-w^{n}(k, t), \widetilde{\beta}_{t^{\prime}}^{n}-\widetilde{\beta}_{t}^{n}, k \in \mathbb{R}, t^{\prime} \in[t, T]\right\}$ is independent of $\mathcal{G}_{t}^{n}$ under $\mathbb{Q}^{n}$. It follows that $\widetilde{\mathbf{\Omega}^{\mathbf{n}}}=\left(\Omega^{n}, \mathcal{G}^{n},\left(\mathcal{G}_{t}^{n}\right)_{t \in[0, T]}, \mathbb{Q}^{n}, z^{n}, w^{n}, \widetilde{\beta}^{n}, \xi^{n}\right)$ is a weak solution to the $\operatorname{SDE}(36)$ with zero drift. By Proposition $26, \mathcal{L}^{\mathbb{Q}^{1}}\left(z^{1}, w^{1}, \widetilde{\beta}^{1}\right)=\mathcal{L}^{\mathbb{Q}^{2}}\left(z^{2}, w^{2}, \widetilde{\beta}^{2}\right)$ and $\mathbb{Q}^{n}$-almost surely, $z^{n}=\mathcal{Z}\left(\xi^{n}, w^{n}, \widetilde{\beta}^{n}\right)$, where $\mathcal{Z}$ is of the form (37).

Moreover, recall that for $n=1,2, \mu_{t}^{n}=\mathcal{L}^{\mathbb{P}^{n}}\left(z_{t}^{n} \mid \mathcal{G}_{t}^{\mu^{n}, W^{n}}\right)$. Recall also that $\mu^{\phi}$ is defined as being the unique fixed-point of $\phi$ in $\mathcal{X}$ (see proof of Proposition 27). Let us state the following lemma, which will be shown at the end of the current proof. 
Lemma 31. Let $n=1,2$. Then $\mathbb{Q}^{n}$-almost surely, for every $t \in[0, T], \mu_{t}^{n}=\mu^{\phi}\left(w^{n}\right)_{t}$. In particular, $\left(\mu_{t}^{n}\right)_{t \in[0, T]}$ is adapted to the completion of $\left(\mathcal{G}_{t}^{W^{n}}\right)_{t \in[0, T]}$, where $\mathcal{G}_{t}^{W^{n}}:=\sigma\left\{w^{n}(k, s) ; k \in\right.$ $\mathbb{R}, s \leqslant t\}$.

Let us consider a measurable function $\psi: \mathcal{C}([0, T], \mathbb{R}) \times \mathcal{C}\left(\mathbb{R} \times[0, T], \mathbb{R}^{2}\right) \rightarrow \mathbb{R}$ such that $\mathbb{E}^{\mathbb{P}^{n}}\left[\left|\psi\left(z^{n}, w^{n}\right)\right|\right]<+\infty$ for $n=1,2$. It follows from (46) and from Lemma 31 that

$$
\begin{aligned}
\mathbb{E}^{\mathbb{P}^{n}}\left[\psi\left(z^{n}, w^{n}\right)\right] & =\mathbb{E}^{\mathbb{Q}^{n}}\left[\psi\left(z^{n}, w^{n}\right) \exp \left(\int_{0}^{T} \widetilde{b}\left(z_{s}^{n}, \mu_{s}^{n}\right) \mathrm{d} \beta_{s}^{n}+\frac{1}{2} \int_{0}^{T}\left|\widetilde{b}\left(z_{s}^{n}, \mu_{s}^{n}\right)\right|^{2} \mathrm{~d} s\right)\right] \\
& =\mathbb{E}^{\mathbb{Q}^{n}}\left[\psi\left(z^{n}, w^{n}\right) \exp \left(\int_{0}^{T} \widetilde{b}\left(z_{s}^{n}, \mu_{s}^{n}\right) \mathrm{d} \widetilde{\beta}_{s}^{n}-\frac{1}{2} \int_{0}^{T}\left|\widetilde{b}\left(z_{s}^{n}, \mu_{s}^{n}\right)\right|^{2} \mathrm{~d} s\right)\right] \\
& =\mathbb{E}^{\mathbb{Q}^{n}}\left[\psi\left(z^{n}, w^{n}\right) \exp \left(\int_{0}^{T} \widetilde{b}\left(z_{s}^{n}, \mu^{\phi}\left(w^{n}\right)_{s}\right) \mathrm{d} \widetilde{\beta}_{s}^{n}-\frac{1}{2} \int_{0}^{T}\left|\widetilde{b}\left(z_{s}^{n}, \mu^{\phi}\left(w^{n}\right)_{s}\right)\right|^{2} \mathrm{~d} s\right)\right] \\
& =\mathbb{E}^{\mathbb{Q}^{n}}\left[\widetilde{\psi}\left(z^{n}, w^{n}, \widetilde{\beta}^{n}\right)\right]
\end{aligned}
$$

where $\widetilde{\psi}$ is a measurable map such that $\mathbb{E}^{\mathbb{Q}^{n}}\left[\left|\widetilde{\psi}\left(z^{n}, w^{n}, \widetilde{\beta}^{n}\right)\right|\right]<+\infty$; the measurability of $\widetilde{\psi}$ follows from the fact that $\mu^{\phi}$ belongs to $\mathcal{X}$. Furthermore, $\mu^{\phi}$ does not depend on $n=1,2$, since it is the unique fixed-point of $\phi$. Recalling the equality $\mathcal{L}^{\mathbb{Q}^{1}}\left(z^{1}, w^{1}, \widetilde{\beta}^{1}\right)=\mathcal{L}^{\mathbb{Q}^{2}}\left(z^{2}, w^{2}, \widetilde{\beta}^{2}\right)$, we conclude that $\mathbb{E}^{\mathbb{P}^{1}}\left[\psi\left(z^{1}, w^{1}\right)\right]=\mathbb{E}^{\mathbb{P}^{2}}\left[\psi\left(z^{2}, w^{2}\right)\right]$. Moreover, by Lemma $31,\left(\mu_{t}^{n}\right)_{t \in[0, T]}$ is $\left(\mathcal{G}_{t}^{W^{n}}\right)_{t \in[0, T]}$-measurable. This completes the proof of Proposition 28.

Proof (Lemma 31). Let us forget about the exponent $n$ in this proof. On the one hand, the process $\left(\mu_{t}\right)_{t \in[0, T]}$ satisfies for every $t \in[0, T], \mu_{t}=\mathcal{L}^{\mathbb{P}}\left(z_{t} \mid \mathcal{G}_{t}^{\mu, W}\right)$ and $(\mu, w)$ is independent of $(\beta, \xi)$. Moreover, it follows from equality (46) that $\mathbb{P}$ is absolutely continuous with respect to $\mathbb{Q}$ with a density given by

$$
\begin{aligned}
\frac{\mathrm{d} \mathbb{P}}{\mathrm{d} \mathbb{Q}} & =\exp \left(\int_{0}^{T} \widetilde{b}\left(z_{s}, \mu_{s}\right) \mathrm{d} \beta_{s}+\frac{1}{2} \int_{0}^{T}\left|\widetilde{b}\left(z_{s}, \mu_{s}\right)\right|^{2} \mathrm{~d} s\right) \\
& =\exp \left(\int_{0}^{T} \widetilde{b}\left(z_{s}, \mu_{s}\right) \mathrm{d} \widetilde{\beta}_{s}-\frac{1}{2} \int_{0}^{T}\left|\widetilde{b}\left(z_{s}, \mu_{s}\right)\right|^{2} \mathrm{~d} s\right) .
\end{aligned}
$$

On the other hand, since $\mu^{\phi}$ is the fixed point of $\phi$, the process $\left(\mu^{\phi}(w)_{t}\right)_{t \in[0, T]}$ satisfies $\mu^{\phi}(w)=\phi\left(\mu^{\phi}\right)(w)=\mathcal{L}^{\mathbb{P}^{\mu^{\phi}, \beta, \xi}(w)}(\mathcal{Z}(\cdot, w, \cdot))$. Since under $\mathbb{Q}, z=\mathcal{Z}(\xi, w, \widetilde{\beta})$, we deduce that for every $t \in[0, T], \mu^{\phi}(w)_{t}=\mathcal{L}^{\mathbb{R}}\left(z_{t} \mid \mathcal{G}_{t}^{W}\right)$, where $\mathcal{G}_{t}^{W}:=\sigma\{w(k, s) ; k \in \mathbb{R}, s \leqslant t\}$ and $\mathbb{R}$ is defined by

$$
\frac{\mathrm{d} \mathbb{R}}{\mathrm{d} \mathbb{Q}}=\exp \left(\int_{0}^{T} \widetilde{b}\left(z_{s}, \mu^{\phi}(w)_{s}\right) \mathrm{d} \widetilde{\beta}_{s}-\frac{1}{2} \int_{0}^{T}\left|\widetilde{b}\left(z_{s}, \mu^{\phi}(w)_{s}\right)\right|^{2} \mathrm{~d} s\right)
$$

Let us prove that

(1) under the probability measure $\mathbb{Q},(\mu, w)$ is independent of $(\widetilde{\beta}, \xi)$;

(2) for every $t \in[0, T], \mathbb{R}$-almost surely, $\mu^{\phi}(w)_{t}=\mathcal{L}^{\mathbb{R}}\left(z_{t} \mid \mathcal{G}_{t}^{\mu, W}\right)$;

(3) conclude the proof of the lemma by comparing, for every $t \in[0, T], \mu_{t}=\mathcal{L}^{\mathbb{P}}\left(z_{t} \mid \mathcal{G}_{t}^{\mu, W}\right)$ with $\mu^{\phi}(w)_{t}=\mathcal{L}^{\mathbb{R}}\left(z_{t} \mid \mathcal{G}_{t}^{\mu, W}\right)$.

Proof of (1). By definition of a weak solution, under probability measure $\mathbb{P}, w, \beta$ and $\xi$ are independent random variables and $(\mu, w)$ is independent of $(\beta, \xi)$. Let us consider bounded and measurable functions $f: \mathbb{R} \rightarrow \mathbb{R}$ and $\psi: \mathcal{C}\left([0, T], \mathcal{P}_{2}(\mathbb{R})\right) \times \mathcal{C}\left(\mathbb{R} \times[0, T], \mathbb{R}^{2}\right) \rightarrow \mathbb{R}$ and let $g:$ 
$[0, T] \rightarrow \mathbb{R}$ be a deterministic square integrable function. Recalling that $\mathrm{d} \widetilde{\beta}_{t}=\mathrm{d} \beta_{t}+\widetilde{b}\left(z_{t}, \mu_{t}\right) \mathrm{d} t$, let us compute

$$
\begin{aligned}
& \mathbb{E}^{\mathbb{Q}}\left[\psi(\mu, w) f(\xi) \exp \left(\int_{0}^{T} g_{s} \mathrm{~d} \widetilde{\beta}_{s}-\frac{1}{2} \int_{0}^{T} g_{s}^{2} \mathrm{~d} s\right)\right] \\
& =\mathbb{E}^{\mathbb{P}}\left[\psi(\mu, w) f(\xi) \exp \left(\int_{0}^{T} g_{s} \mathrm{~d} \widetilde{\beta}_{s}-\frac{1}{2} \int_{0}^{T} g_{s}^{2} \mathrm{~d} s\right) \exp \left(-\int_{0}^{T} \widetilde{b}\left(z_{s}, \mu_{s}\right) \mathrm{d} \beta_{s}-\frac{1}{2} \int_{0}^{T}\left|\widetilde{b}\left(z_{s}, \mu_{s}\right)\right|^{2} \mathrm{~d} s\right)\right] \\
& =\mathbb{E}^{\mathbb{P}}\left[\psi(\mu, w) f(\xi) \exp \left(\int_{0}^{T}\left(g_{s}-\widetilde{b}\left(z_{s}, \mu_{s}\right)\right) \mathrm{d} \beta_{s}-\frac{1}{2} \int_{0}^{T}\left|g_{s}-\widetilde{b}\left(z_{s}, \mu_{s}\right)\right|^{2} \mathrm{~d} s\right)\right] .
\end{aligned}
$$

We now show that the last line is in fact equal to $\mathbb{E}^{\mathbb{P}}[\psi(\mu, w) f(\xi)]$. By expanding the exponential martingale by Itô's formula, it is in fact sufficient to prove that, for any $\left(\mathcal{G}_{t}\right)_{t \in[0, T]}$ progressivelymeasurable and square integrable process $\left(H_{t}\right)_{t \in[0, T]}$, the stochastic integral $\int_{0}^{T} H_{s} d \beta_{s}$ is orthogonal to $\psi(\mu, w) f(\xi)$ under $\mathbb{P}$. By a standard approximation, it is even sufficient to do so for simple processes $\left(H_{t}\right)_{t \in[0, T]}$. In other words, it suffices to prove that, for any $0 \leqslant t \leqslant t^{\prime} \leqslant T$, for any $\mathcal{G}_{t}$-measurable square-integrable random variable $H_{t}$,

$$
\mathbb{E}^{\mathbb{P}}\left[\psi(\mu, w) f(\xi) H_{t}\left(\beta_{t^{\prime}}-\beta_{t}\right)\right]=0 .
$$

By taking the conditional expectation given $\mathcal{G}_{t}$ in the expectation appearing in the left-hand side, it is sufficient to prove that, for any $0 \leqslant t \leqslant t^{\prime} \leqslant T$,

$$
\mathbb{E}^{\mathbb{P}}\left[\psi(\mu, w) f(\xi)\left(\beta_{t^{\prime}}-\beta_{t}\right) \mid \mathcal{G}_{t}\right]=0
$$

Thanks to the compatibility condition in Definition 24,

$$
\mathbb{E}^{\mathbb{P}}\left[\psi(\mu, w) f(\xi)\left(\beta_{t^{\prime}}-\beta_{t}\right) \mid \mathcal{G}_{t}\right]=\mathbb{E}^{\mathbb{P}}\left[\psi(\mu, w) f(\xi) \mid \mathcal{G}_{t}\right] \mathbb{E}^{\mathbb{P}}\left[\left(\beta_{t^{\prime}}-\beta_{t}\right) \mid \mathcal{G}_{t}\right]=0
$$

because $\beta_{t^{\prime}}-\beta_{t}$ is independent of $\mathcal{G}_{t}$. Therefore,

$$
\mathbb{E}^{\mathbb{Q}}\left[\psi(\mu, w) f(\xi) \exp \left(\int_{0}^{T} g_{s} \mathrm{~d} \widetilde{\beta}_{s}-\frac{1}{2} \int_{0}^{T} g_{s}^{2} \mathrm{~d} s\right)\right]=\mathbb{E}^{\mathbb{P}}[\psi(\mu, w) f(\xi)] .
$$

It follows that

$$
\begin{aligned}
\mathbb{E}^{\mathbb{Q}}\left[\psi(\mu, w) f(\xi) \exp \left(\int_{0}^{T} g_{s} \mathrm{~d} \widetilde{\beta}_{s}-\frac{1}{2} \int_{0}^{T} g_{s}^{2} \mathrm{~d} s\right)\right] & =\mathbb{E}^{\mathbb{P}}[\psi(\mu, w)] \cdot \mathbb{E}^{\mathbb{P}}[f(\xi)] \\
= & \mathbb{E}^{\mathbb{Q}}[\psi(\mu, w)] \cdot \mathbb{E}^{\mathbb{Q}}[f(\xi)] \\
= & \mathbb{E}^{\mathbb{Q}}[\psi(\mu, w)] \cdot \mathbb{E}^{\mathbb{Q}}[f(\xi)] \cdot \mathbb{E}^{\mathbb{Q}}\left[\exp \left(\int_{0}^{T} g_{s} \mathrm{~d} \widetilde{\beta}_{s}-\frac{1}{2} \int_{0}^{T} g_{s}^{2} \mathrm{~d} s\right)\right],
\end{aligned}
$$

since $\left(\exp \left(\int_{0}^{T} g_{s} \mathrm{~d} \widetilde{\beta}_{s}-\frac{1}{2} \int_{0}^{T} g_{s}^{2} \mathrm{~d} s\right)\right)_{t \in[0, T]}$ is a martingale under the measure $\mathbb{Q}$. Moreover, the linear span of $\left\{\exp \left(\int_{0}^{T} g_{s} \mathrm{~d} \widetilde{\beta}_{s}\right), g \in L_{2}([0, T], \mathbb{R})\right\}$ is dense in $L_{2}\left(\Omega, \mathcal{G}^{\widetilde{\beta}}, \mathbb{Q}\right)$, where $\mathcal{G}^{\widetilde{\beta}}$ is the $\sigma$ algebra generated by $\left(\widetilde{\beta}_{t}\right)_{t \in[0, T]}$. Therefore, $\left(f(\xi), \exp \left(\int_{0}^{T} g_{s} \mathrm{~d} \widetilde{\beta}_{s}\right)\right)$ generates the $\sigma$-algebra $\mathcal{G}^{\xi, \widetilde{\beta}}$, and thus $(\mu, w)$ and $(\xi, \widetilde{\beta})$ are independent under the probability measure $\mathbb{Q}$.

Proof of (2). Recall that for every $t \in[0, T], \mu^{\phi}(w)_{t}=\mathcal{L}^{\mathbb{R}}\left(z_{t} \mid \mathcal{G}_{t}^{W}\right)$, and let us prove that for every $t \in[0, T], \mathbb{R}$-almost surely, $\mu^{\phi}(w)_{t}=\mathcal{L}^{\mathbb{R}}\left(z_{t} \mid \mathcal{G}_{t}^{\mu, W}\right)$. Let $f: \mathbb{R} \rightarrow \mathbb{R}, g: \mathcal{C}\left([0, T], \mathcal{P}_{2}(\mathbb{R})\right) \rightarrow \mathbb{R}$ and $h: \mathcal{C}\left(\mathbb{R} \times[0, T], \mathbb{R}^{2}\right) \rightarrow \mathbb{R}$ be bounded and measurable functions. Fix $t \in[0, T]$. By (48), we have

$$
\begin{aligned}
\mathbb{E}^{\mathbb{R}}\left[f\left(z_{t}\right) g\right. & (\mu \cdot \wedge t) h(w \cdot \wedge t)] \\
& =\mathbb{E}^{\mathbb{Q}}\left[f\left(z_{t}\right) \exp \left(\int_{0}^{t} \widetilde{b}\left(z_{s}, \mu^{\phi}(w)_{s}\right) \mathrm{d} \widetilde{\beta}_{s}-\frac{1}{2} \int_{0}^{t}\left|\widetilde{b}\left(z_{s}, \mu^{\phi}(w)_{s}\right)\right|^{2} \mathrm{~d} s\right) g(\mu \cdot \wedge t) h(w \cdot \wedge t)\right] \\
& =\mathbb{E}^{\mathbb{Q}}\left[\mathbb{E}^{\mathbb{Q}}\left[F_{t} \mid \mathcal{G}_{t}^{\mu, W}\right] g(\mu \cdot \wedge t) h(w \cdot \wedge t)\right],
\end{aligned}
$$


where (recall that $z$ has the form $z=\mathcal{Z}(\xi, w, \widetilde{\beta})$ )

$$
\begin{aligned}
F_{t} & :=f\left(z_{t}\right) \exp \left(\int_{0}^{t} \widetilde{b}\left(z_{s}, \mu^{\phi}(w)_{s}\right) \mathrm{d} \widetilde{\beta}_{s}-\frac{1}{2} \int_{0}^{t}\left|\widetilde{b}\left(z_{s}, \mu^{\phi}(w)_{s}\right)\right|^{2} \mathrm{~d} s\right) \\
& =f\left(\mathcal{Z}_{t}(\xi, w, \widetilde{\beta})\right) \exp \left(\int_{0}^{t} \widetilde{b}\left(\mathcal{Z}_{s}(\xi, w, \widetilde{\beta}), \mu^{\phi}(w)_{s}\right) \mathrm{d} \widetilde{\beta}_{s}-\frac{1}{2} \int_{0}^{t}\left|\widetilde{b}\left(\mathcal{Z}_{s}(\xi, w, \widetilde{\beta}), \mu^{\phi}(w)_{s}\right)\right|^{2} \mathrm{~d} s\right) .
\end{aligned}
$$

Note that $F_{t}$ is $\mathcal{G}_{t}^{W, \beta, \xi}$-measurable. By statement $(1)$, under probability measure $\mathbb{Q},(\mu, w)$ is independent of $(\widetilde{\beta}, \xi)$. Hence $\mathbb{E}^{\mathbb{Q}}\left[F_{t} \mid \mathcal{G}_{t}^{\mu, W}\right]$ is $\mathcal{G}_{t}^{W}$-measurable. Thus it follows from (49) that

$$
\mathbb{E}^{\mathbb{R}}\left[f\left(z_{t}\right) g(\mu \cdot \wedge t) h(w \cdot \wedge t)\right]=\mathbb{E}^{\mathbb{Q}}\left[\mathbb{E}^{\mathbb{Q}}\left[F_{t} \mid \mathcal{G}_{t}^{\mu, W}\right] \mathbb{E}^{\mathbb{Q}}\left[g(\mu \cdot \wedge t) \mid \mathcal{G}_{t}^{W}\right] h(w \cdot \wedge t)\right] .
$$

Since $\mathbb{E}^{\mathbb{Q}}\left[g(\mu \cdot \wedge t) \mid \mathcal{G}_{t}^{W}\right] h(w \cdot \wedge t)$ is $\mathcal{G}_{t}^{W}$-measurable and bounded, there is a bounded and measurable function $k: \mathcal{C}\left(\mathbb{R} \times[0, T], \mathbb{R}^{2}\right) \rightarrow \mathbb{R}$ such that $\mathbb{E}^{\mathbb{Q}}\left[g(\mu \cdot \wedge t) \mid \mathcal{G}_{t}^{W}\right] h(w \cdot \wedge t)=k(w \cdot \wedge t)$. Thus, redoing the same computations in reverse, we obtain:

$$
\begin{aligned}
\mathbb{E}^{\mathbb{R}}\left[f\left(z_{t}\right) g(\mu \cdot \wedge t) h(w \cdot \wedge t)\right]=\mathbb{E}^{\mathbb{Q}}\left[\mathbb{E}^{\mathbb{Q}}\left[F_{t} \mid \mathcal{G}_{t}^{\mu, W}\right] k(w \cdot \wedge t)\right]=\mathbb{E}^{\mathbb{Q}}\left[F_{t} k(w \cdot \wedge t)\right]=\mathbb{E}^{\mathbb{R}}\left[f\left(z_{t}\right) k(w \cdot \wedge t)\right] \\
=\mathbb{E}^{\mathbb{R}}\left[\int_{\mathbb{R}} f(x) \mathrm{d} \mu^{\phi}(w)_{t}(x) k(w \cdot \wedge t)\right]=\mathbb{E}^{\mathbb{R}}\left[\int_{\mathbb{R}} f(x) \mathrm{d} \mu^{\phi}(w)_{t}(x) \mathbb{E}^{\mathbb{Q}}\left[g(\mu \cdot \wedge t) \mid \mathcal{G}_{t}^{W}\right] h(w \cdot \wedge t)\right] \\
=\mathbb{E}^{\mathbb{R}}\left[\int_{\mathbb{R}} f(x) \mathrm{d} \mu^{\phi}(w)_{t}(x) g(\mu \cdot \wedge t) h(w \cdot \wedge t)\right] .
\end{aligned}
$$

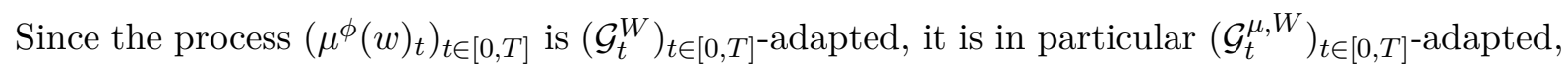
thus equality (50) implies that $\mu^{\phi}(w)_{t}=\mathcal{L}^{\mathbb{R}}\left(z_{t} \mid \mathcal{G}_{t}^{\mu, W}\right)$. It completes the proof of (2).

Proof of (3). Let us denote for every $t \in[0, T], \nu_{t}=\mu^{\phi}(w)_{t}$. We want to prove that $\left(\mu_{t}\right)_{t \in[0, T]}=\left(\nu_{t}\right)_{t \in[0, T]}$. Recall that for every $t \in[0, T], \mathbb{Q}$-almost surely, $\mu_{t}=\mathcal{L}^{\mathbb{P}}\left(z_{t} \mid \mathcal{G}_{t}^{\mu, W}\right)$ and, by point $(2), \nu_{t}=\mathcal{L}^{\mathbb{R}}\left(z_{t} \mid \mathcal{G}_{t}^{\mu, W}\right)$. By (47) and (48),

$$
\begin{aligned}
& \frac{\mathrm{d} \mathbb{P}}{\mathrm{d} \mathbb{Q}}=\exp \left(\int_{0}^{T} \widetilde{b}\left(z_{s}, \mu_{s}\right) \mathrm{d} \widetilde{\beta}_{s}-\frac{1}{2} \int_{0}^{T}\left|\widetilde{b}\left(z_{s}, \mu_{s}\right)\right|^{2} \mathrm{~d} s\right) ; \\
& \frac{\mathrm{d} \mathbb{R}}{\mathrm{d} \mathbb{Q}}=\exp \left(\int_{0}^{T} \widetilde{b}\left(z_{s}, \nu_{s}\right) \mathrm{d} \widetilde{\beta}_{s}-\frac{1}{2} \int_{0}^{T}\left|\widetilde{b}\left(z_{s}, \nu_{s}\right)\right|^{2} \mathrm{~d} s\right) .
\end{aligned}
$$

Let us apply the same computation as in the proof of Lemma 30. Recall that for every $t \in[0, T]$, $\mu^{t}$ denotes $\mathcal{L}^{\mathbb{P}}\left(z \cdot \wedge t \mid \mathcal{G}_{t}^{\mu, W}\right)$ and $\nu^{t}:=\mathcal{L}^{\mathbb{R}}\left(z \cdot \wedge t \mid \mathcal{G}_{t}^{\mu, W}\right)$. For every $t \in[0, T]$,

$$
H\left(\nu^{t} \mid \mu^{t}\right)=\int_{\mathbb{R}} \ln \frac{\mathrm{d} \nu^{t}}{\mathrm{~d} \mu^{t}} \mathrm{~d} \nu^{t}=\mathbb{E}^{\mathbb{R}}\left[\ln \frac{\mathrm{d} \nu^{t}}{\mathrm{~d} \mu^{t}}(z \cdot \wedge t) \mid \mathcal{G}_{t}^{\mu, W}\right] .
$$

We use the fact that under $\mathbb{P}, \beta$ is independent of $(\mu, w)$ in order to prove, exactly as in the proof of Lemma 30 , that for every $t \in[0, T], \mathbb{E}^{\mathbb{P}}\left[\frac{\mathrm{d} \mathbb{R}}{\mathrm{d} \mathbb{P}} \mid \mathcal{G}_{t}^{\mu, W}\right]=1$. Again by mimicking the proof of (43), this leads to

$$
\frac{\mathrm{d} \nu^{t}}{\mathrm{~d} \mu^{t}}(z \cdot \wedge t)=\mathbb{E}^{\mathbb{P}}\left[\frac{\mathrm{d} \mathbb{R}}{\mathrm{dP}} \mid \mathcal{G}_{t}^{z, \mu, W}\right]
$$

Therefore, we finally obtain

$$
H\left(\nu^{t} \mid \mu^{t}\right)=\frac{1}{2} \mathbb{E}^{\mathbb{R}}\left[\int_{0}^{t}\left|\widetilde{b}\left(z_{s}, \nu_{s}\right)-\widetilde{b}\left(z_{s}, \mu_{s}\right)\right|^{2} \mathrm{~d} s \mid \mathcal{G}_{t}^{\mu, W}\right] .
$$


Applying Pinsker's inequality and using the fact that $\widetilde{b}$ is Lipschitz-continuous with respect to the measure variable, we finally obtain for every $t \in[0, T]$,

$$
\mathbb{E}^{\mathbb{Q}}\left[\mathrm{d}_{\mathrm{TV}}\left(\nu_{t}, \mu_{t}\right)^{2}\right] \leqslant \mathbb{E}^{\mathbb{Q}}\left[\mathrm{d}_{\mathrm{TV}}\left(\nu^{t}, \mu^{t}\right)^{2}\right] \leqslant C \int_{0}^{T} \mathbb{E}^{\mathbb{Q}}\left[\mathrm{d}_{\mathrm{TV}}\left(\nu_{s}, \mu_{s}\right)^{2}\right] \mathrm{d} s .
$$

Thus by Gronwall's inequality, we obtain that for every $t \in[0, T], \mathbb{E}^{\mathbb{Q}}\left[\mathrm{d}_{\mathrm{TV}}\left(\nu_{t}, \mu_{t}\right)^{2}\right]=0$. In particular, $\mathbb{Q}$-almost surely, the two continuous processes $\left(\mu_{t}\right)_{t \in[0, T]}$ and $\left(\nu_{t}\right)_{t \in[0, T]}$ are equal. This completes the proof of the lemma.

\section{II.4 Resolution of the SDE when the drift is general}

Let us state the well-posedness result for the general case:

Theorem 32. Let $\eta>0$ and $\delta \in[0,1]$ satisfy the inequality $\eta>\frac{3}{2}(1-\delta)$. Let $b: \mathbb{R} \times \mathcal{P}_{2}(\mathbb{R}) \rightarrow \mathbb{R}$ be of class $\left(H^{\eta}, \mathcal{C}^{\delta}\right)$. Let $f: \mathbb{R} \rightarrow \mathbb{R}$ be a function of order $\alpha \in\left(\frac{3}{2}, \frac{\eta}{1-\delta}\right]$.

Then existence of a weak solution and uniqueness in law hold for equation (35).

Note: The assumption on $\alpha$ is the same as the one given by inequality (34).

As a first step, let us show that a drift function $b$ satisfying the assumptions of Theorem 32 can be written as a sum $\widetilde{b}+(b-\widetilde{b})$, where $\widetilde{b}$ satisfies the assumptions of Proposition 27 and where $b-\widetilde{b}$ satisfies assumptions similar to Definition 12 , and apply on $b-\widetilde{b}$ the same Fourier inversion as in Lemma 15.

Recall that by Definition 23, $b$ can be written as

$$
b(x, \mu)=\int_{\mathbb{R}}\langle k\rangle^{-\eta}\left(\cos (k x) \lambda^{\Re}(k, \mu)+\sin (k x) \lambda^{\Im}(k, \mu)\right) \mathrm{d} k,
$$

where $\lambda=\lambda^{\Re}+i \lambda^{\Im}$ satisfies for every $k \in \mathbb{R}$ and for every $\mu, \nu \in \mathcal{P}_{2}(\mathbb{R})$,

$$
\begin{aligned}
|\lambda(k, \mu)| & \leqslant \Lambda(k) ; \\
|\lambda(k, \mu)-\lambda(k, \nu)| & \leqslant \Lambda(k) \mathrm{d}_{\mathrm{TV}}(\mu, \nu)^{\delta},
\end{aligned}
$$

and $\Lambda$ belongs to $L_{1}(\mathbb{R}) \cap L_{2}(\mathbb{R})$.

Lemma 33. Let $\theta:=\frac{\alpha-\eta}{\delta}$. There exists $\widetilde{\lambda}=\widetilde{\lambda}^{\Re}+i \widetilde{\lambda}^{\Im}$, where $\widetilde{\lambda}^{\Re}, \widetilde{\lambda}^{\Im}: \mathbb{R} \times \mathcal{P}_{2}(\mathbb{R}) \rightarrow \mathbb{R}$, such that for each $k \in \mathbb{R}$ and for each $\mu, \nu \in \mathcal{P}_{2}(\mathbb{R})$,

$$
\begin{aligned}
& |\lambda(k, \mu)-\widetilde{\lambda}(k, \mu)| \leqslant \frac{C}{\langle k\rangle^{\theta \delta}} \Lambda(k) ; \\
& |\widetilde{\lambda}(k, \mu)-\widetilde{\lambda}(k, \nu)| \leqslant C\langle k\rangle^{\theta(1-\delta)} \Lambda(k) \mathrm{d}_{\mathrm{TV}}(\mu, \nu),
\end{aligned}
$$

where $C$ is independent of $k, \mu, \nu$ and $\theta$.

Proof. Let us fix $k \in \mathbb{R}$. We will focus on the proof for the real part; the case of the imaginary part is identical.

Let us define $u: \mathcal{P}_{2}(\mathbb{R}) \rightarrow \mathbb{R}$ by $u(\mu):=\frac{\lambda^{\Re}(k, \mu)}{\Lambda(k)}$. By (52) and (53), for every $\mu, \nu \in \mathcal{P}_{2}(\mathbb{R})$, $|u(\mu)| \leqslant 1$ and $|u(\mu)-u(\nu)| \leqslant \mathrm{d}_{\mathrm{TV}}(\mu, \nu)^{\delta}$. Let $(\Omega, \mathcal{F}, \mathbb{P})$ be a Polish and atomless probability space. Let us define $v: L_{2}(\Omega) \rightarrow \mathbb{R}$ by $v(X):=u(\mathcal{L}(X))$.

The following approximation method is inspired by the inf-convolution techniques. Let $\varepsilon>0$. Let us define $v^{\varepsilon}: L_{2}(\Omega) \rightarrow \mathbb{R}$ by

$$
v^{\varepsilon}(X):=\inf _{Y \in L_{2}(\Omega \times[0,1])}\left\{v(Y)+\frac{1}{2 \varepsilon}\left(\mathbb{P} \otimes \operatorname{Leb}_{[0,1]}\right)[X \neq Y]^{2}\right\} .
$$

We consider here the infimum over random variables in a larger probability space in order to be enseure the existence of a random variable $Y$ independent of $X$. In (56), the map $v$ is extended to $L_{2}(\Omega \in[0,1]) \times \mathbb{R}$ by $v(Y):=u(\mathcal{L}(Y))$. Let us prove that 
(i) $v^{\varepsilon}(X)$ depends only on the law of $X$; thus we can define $u^{\varepsilon}(\mu)$ by letting $u^{\varepsilon}(\mu):=v^{\varepsilon}(X)$, whatever the choice of the random variable $X$ with distribution $\mu$.

(ii) for every $\mu \in \mathcal{P}_{2}(\mathbb{R}),\left|u^{\varepsilon}(\mu)-u(\mu)\right| \leqslant C \varepsilon^{\frac{\delta}{2-\delta}}$.

(iii) for every $\mu, \nu \in \mathcal{P}_{2}(\mathbb{R}),\left|u^{\varepsilon}(\mu)-u^{\varepsilon}(\nu)\right| \leqslant C \varepsilon^{\frac{\delta-1}{2-\delta}} \mathrm{d}_{\mathrm{TV}}(\mu, \nu)$.

Proof of $(i)$. Let $X, X^{\prime} \in L_{2}(\Omega)$ with same law. We want to prove that $v^{\varepsilon}(X)=v^{\varepsilon}\left(X^{\prime}\right)$. Remark that by definition of $v, v(X)$ depends only on the law of $X$. Fix $\eta>0$. There is $Y^{\eta} \in L_{2}(\Omega \times[0,1])$ such that

$$
v\left(Y^{\eta}\right)+\frac{1}{2 \varepsilon}\left(\mathbb{P} \otimes \operatorname{Leb}_{[0,1]}\right)\left[X \neq Y^{\eta}\right]^{2} \leqslant v^{\varepsilon}(X)+\eta .
$$

Let $\nu: \mathbb{R} \times \mathcal{B}(\mathbb{R}) \rightarrow \mathbb{R}$ be the conditional law of $Y^{\eta}$ given $X$; in other words, for every fixed $x \in \mathbb{R}$, $\nu(x, \cdot)$ belongs to $\mathcal{P}_{2}(\mathbb{R})$, for every fixed $A \in \mathcal{B}(\mathbb{R}), x \mapsto \nu(x, A)$ is measurable and for every $f$ : $\mathbb{R}^{2} \rightarrow \mathbb{R}$ bounded and measurable, $\left(\mathbb{E} \otimes \operatorname{Leb}_{[0,1]}\right)\left[f\left(X, Y^{\eta}\right)\right]=\left(\mathbb{E} \otimes \operatorname{Leb}_{[0,1]}\right)\left[\int_{\mathbb{R}} f(X, y) \nu(X, \mathrm{~d} y)\right]$.

Furthermore, for every fixed $x \in \mathbb{R}$, let us denote by $u \in[0,1] \mapsto g(x, u)$ the quantile function associated to the probability measure $\nu(x, \cdot)$. For every $t \in \mathbb{R}$ and for every $u \in[0,1]$, $\{x: g(x, u) \leqslant t\}=\{x: \nu(x,(-\infty, t]) \leqslant u\} \in \mathcal{B}(\mathbb{R})$, so we deduce that for every $u \in[0,1]$, $x \mapsto g(x, u)$ is measurable. Moreover, $u \mapsto g(x, u)$ is a càdlàg function. It follows from [KS91, Proposition 1.13] that $(x, u) \mapsto g(x, u)$ is measurable.

Let $U \in L_{2}([0,1])$ be a random variable with uniform law on $[0,1]$; in particular, it is independent of $X^{\prime}$ (remark that we have considered a larger probability space in order to ensure the existence of $U$ independent of $\left.X^{\prime}\right)$. Let $Y^{\prime}:=g\left(X^{\prime}, U\right)$. Then for every $f: \mathbb{R}^{2} \rightarrow \mathbb{R}$ bounded and measurable

$$
\begin{aligned}
\left(\mathbb{E} \otimes \operatorname{Leb}_{[0,1]}\right)\left[f\left(X^{\prime}, Y^{\prime}\right)\right] & =\left(\mathbb{E} \otimes \operatorname{Leb}_{[0,1]}\right)\left[f\left(X^{\prime}, g\left(X^{\prime}, U\right)\right)\right] \\
& =\left(\mathbb{E} \otimes \operatorname{Leb}_{[0,1]}\right)\left[\int_{0}^{1} f\left(X^{\prime}, g\left(X^{\prime}, u\right)\right) \mathrm{d} u\right] \\
& =\left(\mathbb{E} \otimes \operatorname{Leb}_{[0,1]}\right)\left[\int_{\mathbb{R}} f\left(X^{\prime}, y\right) \nu\left(X^{\prime}, \mathrm{d} y\right)\right] .
\end{aligned}
$$

Since $X$ and $X^{\prime}$ have same law, we deduce that

$$
\left(\mathbb{E} \otimes \operatorname{Leb}_{[0,1]}\right)\left[f\left(X^{\prime}, Y^{\prime}\right)\right]=\left(\mathbb{E} \otimes \operatorname{Leb}_{[0,1]}\right)\left[\int_{\mathbb{R}} f(X, y) \nu(X, \mathrm{~d} y)\right]=\left(\mathbb{E} \otimes \operatorname{Leb}_{[0,1]}\right)\left[f\left(X, Y^{\eta}\right)\right] .
$$

Therefore, the pair $\left(X^{\prime}, Y^{\prime}\right)$ has same distribution as $\left(X, Y^{\eta}\right)$. It follows that

$$
\left(\mathbb{P} \otimes \operatorname{Leb}_{[0,1]}\right)\left[X \neq Y^{\eta}\right]=\left(\mathbb{P} \otimes \operatorname{Leb}_{[0,1]}\right)\left[X^{\prime} \neq Y^{\prime}\right]
$$

and $v\left(Y^{\eta}\right)=v\left(Y^{\prime}\right)$ since $v$ depends only on the law of the random variable. Thus by inequality $(57)$,

$$
v\left(Y^{\prime}\right)+\frac{1}{2 \varepsilon}\left(\mathbb{P} \otimes \operatorname{Leb}_{[0,1]}\right)\left[X^{\prime} \neq Y^{\prime}\right]^{2} \leqslant v^{\varepsilon}(X)+\eta .
$$

By definition $(56)$ of $v^{\varepsilon}, v^{\varepsilon}\left(X^{\prime}\right) \leqslant v\left(Y^{\prime}\right)+\frac{1}{2 \varepsilon}\left(\mathbb{P} \otimes \operatorname{Leb}_{[0,1]}\right)\left[X^{\prime} \neq Y^{\prime}\right]^{2}$, thus $v^{\varepsilon}\left(X^{\prime}\right) \leqslant v^{\varepsilon}(X)+\eta$. We proved that the inequality holds with every $\eta>0$, thus $v^{\varepsilon}\left(X^{\prime}\right) \leqslant v^{\varepsilon}(X)$. By symmetry, $v^{\varepsilon}(X) \leqslant v^{\varepsilon}\left(X^{\prime}\right)$, hence the equality holds true.

Proof of $(i i)$. Let us prove that for every $X \in L_{2}(\Omega),\left|v^{\varepsilon}(X)-v(X)\right| \leqslant C \varepsilon^{\frac{\delta}{2-\delta}}$. Fix $X \in L_{2}(\Omega)$. By definition (56), it is obvious that $v^{\varepsilon}(X) \leqslant v(X)$. Thus it is sufficient to prove that $v(X)-v^{\varepsilon}(X) \leqslant C \varepsilon^{\frac{\delta}{2-\delta}}$. 
Fix $\eta>0$. There exists $Y^{\eta}$ such that (57). It follows that

$$
v\left(Y^{\eta}\right)+\frac{1}{2 \varepsilon}\left(\mathbb{P} \otimes \operatorname{Leb}_{[0,1]}\right)\left[X \neq Y^{\eta}\right]^{2} \leqslant v(X)+\eta .
$$

By definition of $v,\left|v(X)-v\left(Y^{\eta}\right)\right|=\left|u(\mathcal{L}(X))-u\left(\mathcal{L}\left(Y^{\eta}\right)\right)\right| \leqslant \mathrm{d}_{\mathrm{TV}}\left(\mathcal{L}(X), \mathcal{L}\left(Y^{\eta}\right)\right)^{\delta}$. Therefore, by $(33)$,

$$
\mathrm{d}_{\mathrm{TV}}\left(\mathcal{L}(X), \mathcal{L}\left(Y^{\eta}\right)\right)^{2} \leqslant 4\left(\mathbb{P} \otimes \operatorname{Leb}_{[0,1]}\right)\left[X \neq Y^{\eta}\right]^{2} \leqslant 8 \varepsilon\left[\mathrm{d}_{\mathrm{TV}}\left(\mathcal{L}(X), \mathcal{L}\left(Y^{\eta}\right)\right)^{\delta}+\eta\right] .
$$

Let $l:=\lim \sup _{\eta \searrow 0} \mathrm{~d}_{\mathrm{TV}}\left(\mathcal{L}(X), \mathcal{L}\left(Y^{\eta}\right)\right)$. Thus $l^{2} \leqslant 8 \varepsilon l^{\delta}$, hence we get $l^{2-\delta} \leqslant 8 \varepsilon$. It follows that

$$
\limsup _{\eta \searrow 0} \mathrm{~d}_{\mathrm{TV}}\left(\mathcal{L}(X), \mathcal{L}\left(Y^{\eta}\right)\right) \leqslant 2^{\frac{3}{2-\delta}} \varepsilon^{\frac{1}{2-\delta}} .
$$

By inequality (57),

$$
\begin{aligned}
v(X)-v^{\varepsilon}(X) & \leqslant v(X)-v\left(Y^{\eta}\right)-\frac{1}{2 \varepsilon}\left(\mathbb{P} \otimes \operatorname{Leb}_{[0,1]}\right)\left[X \neq Y^{\eta}\right]^{2}+\eta \\
& \leqslant\left|v(X)-v\left(Y^{\eta}\right)\right|+\eta \leqslant \mathrm{d}_{\mathrm{TV}}\left(\mathcal{L}(X), \mathcal{L}\left(Y^{\eta}\right)\right)^{\delta}+\eta
\end{aligned}
$$

By passing to the limit $\eta \searrow 0$, we obtain $v(X)-v^{\varepsilon}(X) \leqslant C \varepsilon^{\frac{\delta}{2-\delta}}$, which completes the proof of (ii).

Proof of $(i i i)$. Let us first prove that $u^{\varepsilon}$ is also $\delta$-Hölder continuous. Let $\mu, \nu \in \mathcal{P}_{2}(\mathbb{R})$. Let $X$ and $X^{\prime} \in L_{2}(\Omega)$ with respective distributions $\mu$ and $\nu$. Fix $\eta>0$. Let $Y^{\eta}$ satisfying (57). Then

$$
\begin{aligned}
& v^{\varepsilon}\left(X^{\prime}\right)-v^{\varepsilon}(X) \leqslant v^{\varepsilon}\left(X^{\prime}\right)-v\left(Y^{\eta}\right)-\frac{1}{2 \varepsilon}\left(\mathbb{P} \otimes \operatorname{Leb}_{[0,1]}\right)\left[X \neq Y^{\eta}\right]^{2}+\eta \\
& \leqslant v\left(Y^{\eta}+X^{\prime}-X\right)+\frac{1}{2 \varepsilon}\left(\mathbb{P} \otimes \operatorname{Leb}_{[0,1]}\right)\left[X^{\prime} \neq Y^{\eta}+X^{\prime}-X\right]^{2} \\
& \quad-v\left(Y^{\eta}\right)-\frac{1}{2 \varepsilon}\left(\mathbb{P} \otimes \operatorname{Leb}_{[0,1]}\right)\left[X \neq Y^{\eta}\right]^{2}+\eta \\
& \leqslant\left|v\left(Y^{\eta}+X^{\prime}-X\right)-v\left(Y^{\eta}\right)\right|+\eta \leqslant \operatorname{d}_{\mathrm{TV}}\left(\mathcal{L}\left(Y^{\eta}+X^{\prime}-X\right), \mathcal{L}\left(Y^{\eta}\right)\right)^{\delta}+\eta .
\end{aligned}
$$

By definition of the distance in total variation, $\mathrm{d}_{\mathrm{TV}}\left(\mathcal{L}\left(Y^{\eta}+X^{\prime}-X\right), \mathcal{L}\left(Y^{\eta}\right)\right) \leqslant 2 \mathbb{P}\left[X^{\prime} \neq X\right]$. Thus for every $\eta>0, v^{\varepsilon}\left(X^{\prime}\right)-v^{\varepsilon}(X) \leqslant 2^{\delta} \mathbb{P}\left[X^{\prime} \neq X\right]^{\delta}+\eta$. By letting $\eta$ tend to zero and by symmetry, we deduce that there is $C>0$ depending only on $\delta$ such that

$$
\left|u^{\varepsilon}(\mu)-u^{\varepsilon}(\nu)\right|=\left|v^{\varepsilon}(X)-v^{\varepsilon}\left(X^{\prime}\right)\right| \leqslant C \mathbb{P}\left[X^{\prime} \neq X\right]^{\delta} .
$$

By taking the infimum over every coupling $\left(X, X^{\prime}\right)$ of $(\mu, \nu)$, we finally get

$$
\left|u^{\varepsilon}(\mu)-u^{\varepsilon}(\nu)\right| \leqslant C \mathrm{~d}_{\mathrm{TV}}(\mu, \nu)^{\delta} .
$$

Therefore, $u^{\varepsilon}$ is also $\delta$-Hölder continuous.

Keep $X, X^{\prime} \in L_{2}(\Omega)$ two random variables with laws $\mu$ and $\nu$. Let $\left(Y^{\eta}\right)_{\eta>0}$ satisfy (57). It follows from (58) and (59) that

$$
\limsup _{\eta \searrow 0}\left(\mathbb{P} \otimes \operatorname{Leb}_{[0,1]}\right)\left[X \neq Y^{\eta}\right] \leqslant \sqrt{2 \varepsilon 2^{\frac{3 \delta}{2-\delta}} \varepsilon^{\frac{\delta}{2-\delta}}}=2^{\frac{1+\delta}{2-\delta}} \varepsilon^{\frac{1}{2-\delta}} .
$$

For every $\eta>0$, let us define

$$
\begin{array}{r}
S^{\eta}:=\left\{Y \in L_{2}(\Omega \times[0,1]):\left(\mathbb{P} \otimes \operatorname{Leb}_{[0,1]}\right)[X \neq Y] \leqslant 2^{\frac{1+\delta}{2-\delta}} \varepsilon^{\frac{1}{2-\delta}}+\eta\right. \\
\text { or } \left.\quad\left(\mathbb{P} \otimes \operatorname{Leb}_{[0,1]}\right)\left[X^{\prime} \neq Y\right] \leqslant 2^{\frac{1+\delta}{2-\delta}} \varepsilon^{\frac{1}{2-\delta}}+\eta\right\} .
\end{array}
$$


Fix $\eta>0$. Thus there is $Y^{\eta} \in S^{\eta}$ such that (57) holds true. We deduce that

$$
\begin{aligned}
v^{\varepsilon}\left(X^{\prime}\right)-v^{\varepsilon}(X) \leqslant & v\left(Y^{\eta}\right)+\frac{1}{2 \varepsilon}\left(\mathbb{P} \otimes \operatorname{Leb}_{[0,1]}\right)\left[X^{\prime} \neq Y^{\eta}\right]^{2} \\
& -v\left(Y^{\eta}\right)-\frac{1}{2 \varepsilon}\left(\mathbb{P} \otimes \operatorname{Leb}_{[0,1]}\right)\left[X \neq Y^{\eta}\right]^{2}+\eta \\
\leqslant & \frac{1}{2 \varepsilon}\left(\left(\mathbb{P} \otimes \operatorname{Leb}_{[0,1]}\right)\left[X^{\prime} \neq Y^{\eta}\right]^{2}-\left(\mathbb{P} \otimes \operatorname{Leb}_{[0,1]}\right)\left[X \neq Y^{\eta}\right]^{2}\right)+\eta .
\end{aligned}
$$

By symmetry, we deduce that

$$
\left|v^{\varepsilon}\left(X^{\prime}\right)-v^{\varepsilon}(X)\right| \leqslant \frac{1}{2 \varepsilon} \sup _{Y \in S^{\eta}}\left|\left(\mathbb{P} \otimes \operatorname{Leb}_{[0,1]}\right)\left[X^{\prime} \neq Y\right]^{2}-\left(\mathbb{P} \otimes \operatorname{Leb}_{[0,1]}\right)[X \neq Y]^{2}\right|+\eta .
$$

Moreover,

$$
\begin{aligned}
\mid\left(\mathbb{P} \otimes \operatorname{Leb}_{[0,1]}\right)\left[X^{\prime} \neq Y\right]^{2}- & \left(\mathbb{P} \otimes \operatorname{Leb}_{[0,1]}\right)[X \neq Y]^{2} \mid \\
\leqslant & \left|\left(\mathbb{P} \otimes \operatorname{Leb}_{[0,1]}\right)\left[X^{\prime} \neq Y\right]-\left(\mathbb{P} \otimes \operatorname{Leb}_{[0,1]}\right)[X \neq Y]\right| \\
& \cdot\left|\left(\mathbb{P} \otimes \operatorname{Leb}_{[0,1]}\right)\left[X^{\prime} \neq Y\right]+\left(\mathbb{P} \otimes \operatorname{Leb}_{[0,1]}\right)[X \neq Y]\right| \\
\leqslant & \mathbb{P}\left[X^{\prime} \neq X\right] \cdot\left|\left(\mathbb{P} \otimes \operatorname{Leb}_{[0,1]}\right)\left[X^{\prime} \neq Y\right]+\left(\mathbb{P} \otimes \operatorname{Leb}_{[0,1]}\right)[X \neq Y]\right| .
\end{aligned}
$$

For every $Y \in S^{\eta}$, we have

$$
\left|\left(\mathbb{P} \otimes \operatorname{Leb}_{[0,1]}\right)\left[X^{\prime} \neq Y\right]+\left(\mathbb{P} \otimes \operatorname{Leb}_{[0,1]}\right)[X \neq Y]\right| \leqslant \mathbb{P}\left[X \neq X^{\prime}\right]+2\left(2^{\frac{1+\delta}{2-\delta}} \varepsilon^{\frac{1}{2-\delta}}+\eta\right) .
$$

By passing to the limit $\eta \searrow 0$ it follows that there exists $C>0$ depending on $\delta$ such that for every $X, X^{\prime} \in L_{2}(\Omega)$ with respective distributions $\mu$ and $\nu$,

$$
\left|u^{\varepsilon}(\mu)-u^{\varepsilon}(\nu)\right|=\left|v^{\varepsilon}(X)-v^{\varepsilon}\left(X^{\prime}\right)\right| \leqslant \frac{1}{2 \varepsilon} \mathbb{P}\left[X^{\prime} \neq X\right]\left(\mathbb{P}\left[X^{\prime} \neq X\right]+C \varepsilon^{\frac{1}{2-\delta}}\right) .
$$

Let us distinguish two cases:

- if $\mathrm{d}_{\mathrm{TV}}(\mu, \nu)<\varepsilon^{\frac{1}{2-\delta}}$ : by definition (33), there exists a coupling $\left(X, X^{\prime}\right)$ of law $(\mu, \nu)$ such that $\mathbb{P}\left[X \neq X^{\prime}\right]<\varepsilon^{\frac{1}{2-\delta}}$. Thus

$$
\left|u^{\varepsilon}(\mu)-u^{\varepsilon}(\nu)\right| \leqslant C \frac{\varepsilon^{\frac{1}{2-\delta}}}{\varepsilon} \mathbb{P}\left[X^{\prime} \neq X\right] \leqslant C \varepsilon^{\frac{\delta-1}{2-\delta}} \mathrm{d}_{\mathrm{TV}}(\mu, \nu) .
$$

- if $\mathrm{d}_{\mathrm{TV}}(\mu, \nu) \geqslant \varepsilon^{\frac{1}{2-\delta}}$ : recall that $u^{\varepsilon}$ is $\delta$-Hölder continuous (see (60)). Thus

$$
\left|u^{\varepsilon}(\mu)-u^{\varepsilon}(\nu)\right| \leqslant C \mathrm{~d}_{\mathrm{TV}}(\mu, \nu)^{\delta} \leqslant C \frac{\mathrm{d}_{\mathrm{TV}}(\mu, \nu)}{\mathrm{d}_{\mathrm{TV}}(\mu, \nu)^{1-\delta}} \leqslant C \varepsilon^{\frac{\delta-1}{2-\delta}} \mathrm{d}_{\mathrm{TV}}(\mu, \nu) .
$$

This completes the proof of (iii).

Let us conclude the proof of Lemma 33. Let us define $\widetilde{\lambda}^{\Re}(k, \mu):=\Lambda(k) u^{\varepsilon}(\mu)$, with $\varepsilon=$ $\frac{1}{\langle k\rangle^{\theta(2-\delta)}}$. For every $\mu, \nu \in \mathcal{P}_{2}(\mathbb{R})$, we have

$$
\begin{aligned}
& \left|\widetilde{\lambda}^{\Re}(k, \mu)-\lambda^{\Re}(k, \mu)\right| \leqslant \Lambda(k)\left|u^{\varepsilon}(\mu)-u(\mu)\right| \leqslant C \Lambda(k) \varepsilon^{\frac{\delta}{2-\delta}} \leqslant C \Lambda(k) \frac{1}{\langle k\rangle^{\theta \delta}} \\
& \left|\widetilde{\lambda}^{\Re}(k, \mu)-\widetilde{\lambda}^{\Re}(k, \nu)\right| \leqslant \Lambda(k)\left|u^{\varepsilon}(\mu)-u^{\varepsilon}(\nu)\right| \leqslant C \Lambda(k) \varepsilon^{\frac{\delta-1}{2-\delta}} \mathrm{d}_{\mathrm{TV}}(\mu, \nu) \leqslant C \Lambda(k)\langle k\rangle^{\theta(1-\delta)} \mathrm{d}_{\mathrm{TV}}(\mu, \nu) .
\end{aligned}
$$

It completes the proof of (54) and (55) for the case of the real part. The proof for the imaginary part is the same. 
In particular, it follows from (52) and from (54) that there is $C>0$ such that for each $k \in \mathbb{R}$, $|\widetilde{\lambda}(k, \cdot)| \leqslant C \Lambda(k)$.

Let us define

$$
\widetilde{b}(x, \mu):=\int_{\mathbb{R}}\langle k\rangle^{-\eta}\left(\cos (k x) \widetilde{\lambda}^{\Re}(k, \mu)+\sin (k x) \widetilde{\lambda}^{\Im}(k, \mu)\right) \mathrm{d} k .
$$

For every $x \in \mathbb{R}$ and $\mu \in \mathcal{P}_{2}(\mathbb{R})$,

$$
|\widetilde{b}(x, \mu)| \leqslant C \int_{\mathbb{R}}\langle k\rangle^{-\eta}\left(\left|\widetilde{\lambda}^{\Re}(k, \mu)\right|+\left|\widetilde{\lambda}^{\Im}(k, \mu)\right|\right) \mathrm{d} k \leqslant C \int_{\mathbb{R}}\langle k\rangle^{-\eta} \Lambda(k) \mathrm{d} k \leqslant C,
$$

since $\eta>0$ and $\Lambda \in L_{1}(\mathbb{R})$. Furthermore, by (55), for every $x \in \mathbb{R}$ and for every $\mu, \nu \in \mathcal{P}_{2}(\mathbb{R})$,

$$
\begin{aligned}
|\widetilde{b}(x, \mu)-\widetilde{b}(x, \nu)| & \leqslant \int_{\mathbb{R}}\langle k\rangle^{-\eta}\left(\left|\widetilde{\lambda}^{\Re}(k, \mu)-\widetilde{\lambda}^{\Re}(k, \nu)\right|+\left|\widetilde{\lambda}^{\Im}(k, \mu)-\widetilde{\lambda}^{\Im}(k, \nu)\right|\right) \mathrm{d} k \\
& \leqslant C \int_{\mathbb{R}}\langle k\rangle^{-\eta}\langle k\rangle^{\theta(1-\delta)} \Lambda(k) \mathrm{d} k \mathrm{~d}_{\mathrm{TV}}(\mu, \nu) .
\end{aligned}
$$

Moreover, $\eta-\theta(1-\delta) \geqslant 0$. Indeed, $\eta-\theta(1-\delta)=\eta+\theta \delta-\theta=\alpha-\frac{\alpha-\eta}{\delta}=\frac{\eta-\alpha(1-\delta)}{\delta} \geqslant 0$ by inequality (34). Since $\Lambda$ belongs to $L_{1}(\mathbb{R})$, it implies that $\int_{\mathbb{R}}\langle k\rangle^{-\eta}\langle k\rangle^{\theta(1-\delta)} \Lambda(k) \mathrm{d} k<+\infty$. Therefore, the drift function $\widetilde{b}$ is uniformly bounded and uniformly Lipschitz-continuous in the measure variable.

\section{II.4.1 Existence of a weak solution to the SDE with drift function $b$.}

Let us prove existence of a weak solution to equation (35). We follow the same idea as in Theorem 17.

Proof (Theorem 32, existence part). Let $\boldsymbol{\Omega}$ be a weak solution to the SDE (38) with $\operatorname{drift} \widetilde{b}$ given by (61). In particular, $\mathbb{P}$-almost surely and for every $t \in[0, T]$,

$$
z_{t}=\xi+\int_{0}^{t} \int_{\mathbb{R}} f(k) \Re\left(e^{-i k z_{s}} \mathrm{~d} w(k, s)\right)+\beta_{t}+\int_{0}^{t} \widetilde{b}\left(z_{s}, \mu_{s}\right) \mathrm{d} s,
$$

where for every $t \in[0, T], \mu_{t}=\mathcal{L}^{\mathbb{P}}\left(z_{t} \mid \mathcal{G}_{t}^{W}\right)$ and $\mathcal{G}_{t}^{W}:=\sigma\{w(k, s), k \in \mathbb{R}, s \leqslant t\}$. Recall that Proposition 28 states that every weak solution has this form, i.e. $\left(\mu_{t}\right)_{t \in[0, T]}$ is adapted to (the completion of) $\left(\mathcal{G}_{t}^{W}\right)_{t \in[0, T]}$.

Let $\left(h_{t}\right)_{t \in[0, T]}=\left(h_{t}^{\Re}+i h_{t}^{\Im}\right)_{t \in[0, T]}$ be a process with values in $L_{2}(\mathbb{R}, \mathbb{C})$ satisfying for every $t \in[0, T]$ and for every $x \in \mathbb{R}$,

$$
(b-\widetilde{b})\left(x, \mu_{t}\right)=\int_{\mathbb{R}} f(k)\left(\cos (k x) h_{t}^{\Re}(k)+\sin (k x) h_{t}^{\Im}(k)\right) \mathrm{d} k .
$$

By (51) and (61), the unique solution to (62) is given, for every $k \in \mathbb{R}$ and for every $t \in[0, T]$, by

$$
h_{t}(k)=\frac{1}{f(k)}\langle k\rangle^{-\eta}\left(\lambda\left(k, \mu_{t}\right)-\widetilde{\lambda}\left(k, \mu_{t}\right)\right) .
$$

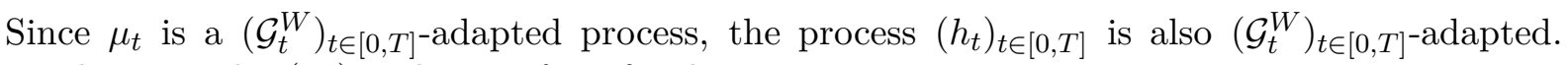
Furthermore, by (54) and since $f$ is of order $\alpha$,

$$
\begin{aligned}
\int_{0}^{T} \int_{\mathbb{R}}\left|h_{t}(k)\right|^{2} \mathrm{~d} k \mathrm{~d} t & \leqslant C \int_{0}^{T} \int_{\mathbb{R}}\langle k\rangle^{2 \alpha-2 \eta}\left|\lambda\left(k, \mu_{t}\right)-\widetilde{\lambda}\left(k, \mu_{t}\right)\right|^{2} \mathrm{~d} k \mathrm{~d} t \\
& \leqslant C \int_{0}^{T} \int_{\mathbb{R}}\langle k\rangle^{2 \alpha-2 \eta-2 \theta \delta} \Lambda(k)^{2} \mathrm{~d} k \mathrm{~d} t .
\end{aligned}
$$


Since $\alpha=\eta+\theta \delta$ and $\Lambda \in L_{2}(\mathbb{R})$, we deduce that $\int_{0}^{T} \int_{\mathbb{R}}\left|h_{t}(k)\right|^{2} \mathrm{~d} k \mathrm{~d} t$ is bounded by a deterministic constant. Therefore, the measure $\mathbb{Q}$ on $(\Omega, \mathcal{G})$ with the following density with respect to $\mathbb{P}$ :

$$
\frac{\mathrm{d} \mathbb{Q}}{\mathrm{d} \mathbb{P}}=\exp \left(\int_{0}^{T} \int_{\mathbb{R}} h_{t}^{\Re}(k) \mathrm{d} w^{\Re}(k, t)+\int_{0}^{T} \int_{\mathbb{R}} h_{t}^{\Im}(k) \mathrm{d} w^{\Im}(k, t)-\frac{1}{2} \int_{0}^{T} \int_{\mathbb{R}}\left|h_{t}(k)\right|^{2} \mathrm{~d} k \mathrm{~d} t\right)
$$

is a probability measure. Let us define $\widetilde{w}(k, t)=\widetilde{w}^{\Re}(k, t)+i \widetilde{w}^{\Im}(k, t)$, where

$$
\begin{aligned}
& \widetilde{w}^{\Re}(k, t):=w^{\Re}(k, t)-\int_{0}^{t} \int_{0}^{k} h_{s}^{\Re}(l) \mathrm{d} l \mathrm{~d} s, \\
& \widetilde{w}^{\Im}(k, t):=w^{\Im}(k, t)-\int_{0}^{t} \int_{0}^{k} h_{s}^{\Im}(l) \mathrm{d} l \mathrm{~d} s .
\end{aligned}
$$

By Girsanov's Theorem, $\mathcal{L}^{\mathbb{Q}}(\widetilde{w}, \beta, \xi)=\mathcal{L}^{\mathbb{P}}(w, \beta, \xi)$ and for any $t \in[0, T]$, the $\sigma$-field $\sigma\left\{\widetilde{w}\left(k, t^{\prime}\right)-\right.$ $\left.\widetilde{w}(k, t), \beta_{t^{\prime}}-\beta_{t}, k \in \mathbb{R}, t^{\prime} \in[t, T]\right\}$ is independent of $\mathcal{G}_{t}$ under $\mathbb{Q}$. Moreover, $\mathbb{Q}$-almost surely, the process $\left(z_{t}\right)_{t \in[0, T]}$ satisfies

$$
\begin{aligned}
z_{t}=\xi & +\int_{0}^{t} \int_{\mathbb{R}} f(k) \Re\left(e^{-i k z_{s}} \mathrm{~d} \widetilde{w}(k, s)\right)+\int_{0}^{t} \int_{\mathbb{R}} f(k)\left(\cos \left(k z_{s}\right) h_{t}^{\Re}(k)+\sin \left(k z_{s}\right) h_{t}^{\Im}(k)\right) \mathrm{d} k \mathrm{~d} s \\
& +\beta_{t}+\int_{0}^{t} \widetilde{b}\left(z_{s}, \mu_{s}\right) \mathrm{d} s \\
=\xi & +\int_{0}^{t} \int_{\mathbb{R}} f(k) \Re\left(e^{-i k z_{s}} \mathrm{~d} \widetilde{w}(k, s)\right)+\int_{0}^{t}(b-\widetilde{b})\left(z_{s}, \mu_{s}\right) \mathrm{d} s+\beta_{t}+\int_{0}^{t} \widetilde{b}\left(z_{s}, \mu_{s}\right) \mathrm{d} s \\
=\xi & +\int_{0}^{t} \int_{\mathbb{R}} f(k) \Re\left(e^{-i k z_{s}} \mathrm{~d} \widetilde{w}(k, s)\right)+\beta_{t}+\int_{0}^{t} b\left(z_{s}, \mu_{s}\right) \mathrm{d} s .
\end{aligned}
$$

Furthermore, recall that for every $t \in[0, T] \mathbb{P}$-almost surely, $\mu_{t}=\mathcal{L}^{\mathbb{P}}\left(z_{t} \mid \mathcal{G}_{t}^{W}\right)$. We want to prove that for every $t \in[0, T] \mathbb{Q}$-almost surely, $\mu_{t}=\mathcal{L}^{\mathbb{Q}}\left(z_{t} \mid \mathcal{G}_{t}^{\mu, \widetilde{W}}\right)$, where the filtration $\left(\mathcal{G}_{t}^{\mu, \widetilde{W}}\right)_{t \in[0, T]}$ is defined by $\mathcal{G}_{t}^{\mu, \widetilde{W}}=\sigma\left\{\widetilde{w}(k, s), \mu_{s} ; k \in \mathbb{R}, s \leqslant t\right\}$. Let $\psi: \mathbb{R} \rightarrow \mathbb{R}$ and $\varphi:$ $\mathcal{C}\left([0, T], \mathcal{P}_{2}(\mathbb{R})\right) \times \mathcal{C}\left(\mathbb{R} \times[0, T], \mathbb{R}^{2}\right) \rightarrow \mathbb{R}$ be bounded and measurable functions. Fix $t \in[0, T]$. Then

$$
\begin{aligned}
& \mathbb{E}^{\mathbb{Q}}\left[\psi\left(z_{t}\right) \varphi(\mu \cdot \wedge t, \widetilde{w} \cdot \wedge t)\right] \\
& \quad=\mathbb{E}^{\mathbb{P}}\left[\psi\left(z_{t}\right) \varphi(\mu \cdot \wedge t, \widetilde{w} \cdot \wedge t) \exp \left(\int_{0}^{t} \int_{\mathbb{R}} \Re\left(\overline{h_{t}(k)} \mathrm{d} w(k, t)\right)-\frac{1}{2} \int_{0}^{t} \int_{\mathbb{R}}\left|h_{t}(k)\right|^{2} \mathrm{~d} k \mathrm{~d} t\right)\right] .
\end{aligned}
$$

Recall that the process $\left(h_{t}\right)_{t \in[0, T]}$ is $\left(\mathcal{G}_{t}^{W}\right)_{t \in[0, T]}$-adapted. It follows that the process $\left(\widetilde{w}_{\cdot \wedge t}\right)_{t \in[0, T]}$ is also $\left(\mathcal{G}_{t}^{W}\right)_{t \in[0, T]}$-adapted, since $\widetilde{w} \cdot \wedge t=w \cdot \wedge t-\int_{0}^{\cdot \wedge t} \int_{0}^{\cdot} h_{s}(l) \mathrm{d} l \mathrm{~d} s$. Thus

$$
\begin{aligned}
& \mathbb{E}^{\mathbb{Q}}\left[\psi\left(z_{t}\right) \varphi\left(\mu \cdot \wedge t, \widetilde{w}_{\cdot \wedge t}\right)\right] \\
& =\mathbb{E}^{\mathbb{P}}\left[\mathbb{E}^{\mathbb{P}}\left[\psi\left(z_{t}\right) \mid \mathcal{G}_{t}^{W}\right] \varphi(\mu \cdot \wedge t, \widetilde{w} \cdot \wedge t) \exp \left(\int_{0}^{t} \int_{\mathbb{R}} \Re\left(\overline{h_{t}(k)} \mathrm{d} w(k, t)\right)-\frac{1}{2} \int_{0}^{t} \int_{\mathbb{R}}\left|h_{t}(k)\right|^{2} \mathrm{~d} k \mathrm{~d} t\right)\right] \\
& =\mathbb{E}^{\mathbb{P}}\left[\int_{\mathbb{R}} \psi(x) \mathrm{d} \mu_{t}(x) \varphi\left(\mu \cdot \wedge t, \widetilde{w}_{\cdot \wedge t}\right) \exp \left(\int_{0}^{t} \int_{\mathbb{R}} \Re\left(\overline{h_{t}(k)} \mathrm{d} w(k, t)\right)-\frac{1}{2} \int_{0}^{t} \int_{\mathbb{R}}\left|h_{t}(k)\right|^{2} \mathrm{~d} k \mathrm{~d} t\right)\right] \\
& =\mathbb{E}^{\mathbb{Q}}\left[\int_{\mathbb{R}} \psi(x) \mathrm{d} \mu_{t}(x) \varphi(\mu \cdot \wedge t, \widetilde{w} \cdot \wedge t)\right] .
\end{aligned}
$$

Therefore, for every $t \in[0, T], \mathbb{E}^{\mathbb{Q}}\left[\psi\left(z_{t}\right) \mid \mathcal{G}_{t}^{\mu, \widetilde{W}}\right]=\int_{\mathbb{R}} \psi(x) \mathrm{d} \mu_{t}(x)$. We deduce that for every $t \in[0, T], \mathbb{Q}$-almost surely $\mu_{t}=\mathcal{L}^{\mathbb{Q}}\left(z_{t} \mid \mathcal{G}_{t}^{\mu, \widetilde{W}}\right)$. 
Furthermore, the pair $(\mu, \widetilde{w})$ is $\mathcal{G}^{W}$-measurable and, subsequently, $\frac{d \mathbb{Q}}{d \mathbb{P}}$ is also $\mathcal{G}^{W}$-measurable. By independence of $(\xi, w, \beta)$ under $\mathbb{P}$, we deduce that $(\mu, \widetilde{w})$ and $(\beta, \xi)$ are independent under $\mathbb{Q}$. By the same argument and by the compatibility property under $\mathbb{P}$, we deduce that, under $\mathbb{Q}$, for any $t \in[0, T],(\xi, \widetilde{w}, \mu)$ and $\beta$ are conditionally independent given $\mathcal{G}_{t}$, which is the required compatibility condition.

Therefore $\left(\Omega, \mathcal{G},\left(\mathcal{G}_{t}\right)_{t \in[0, T]}, \mathbb{Q}, z, \widetilde{w}, \beta, \xi\right)$ is a weak solution to (35). This proves the first statement of Theorem 32 .

Remark 34. In Remark 29, we emphasized the importance of the filtration under which $\left(\mu_{t}\right)_{t \in[0, T]}$ is adapted. It makes sense in (63), because in order to identify $\int_{\mathbb{R}} \psi(x) \mathrm{d} \mu_{t}(x)$ with the conditional expectation $\mathbb{E}^{\mathbb{Q}}\left[\psi\left(z_{t}\right) \mid \mathcal{G}_{t}^{\mu, \widetilde{W}}\right]$, we need to know that $\mu_{t}$ is $\mathcal{G}_{t}^{\mu, \widetilde{W}}$-measurable. This is obviously true, but it is not necessarily true with $\mathcal{G}_{t}^{\widetilde{W}}$ instead of $\mathcal{G}_{t}^{\mu, \widetilde{W}}$.

\section{II.4.2 Uniqueness in law for the SDE with drift function $b$.}

Let us conclude the proof of Theorem 32 by showing uniqueness in law for equation (35). We follow the same idea as in Theorem 17.

Proof (Theorem 32, uniqueness part). Let $\boldsymbol{\Omega}^{\mathbf{1}}$ and $\boldsymbol{\Omega}^{\mathbf{2}}$ be two weak solutions to (35). We want to prove that $\mathcal{L}^{\mathbb{P}^{1}}\left(z^{1}\right)=\mathcal{L}^{\mathbb{P}^{2}}\left(z^{2}\right)$. In particular, for $n=1,2, \mathbb{P}^{n}$-almost surely, the process $\left(z_{t}^{n}\right)_{t \in[0, T]}$ satisfies

$$
z_{t}^{n}=\xi^{n}+\int_{0}^{t} \int_{\mathbb{R}} f(k) \Re\left(e^{-i k z_{s}^{n}} \mathrm{~d} w^{n}(k, s)\right)+\beta_{t}^{n}+\int_{0}^{t} b\left(z_{s}^{n}, \mu_{s}^{n}\right) \mathrm{d} s,
$$

where for every $t \in[0, T], \mu_{t}^{n}=\mathcal{L}^{\mathbb{P}^{n}}\left(z_{t}^{n} \mid \mathcal{G}_{t}^{\mu^{n}, W^{n}}\right), \mathcal{G}_{t}^{\mu^{n}, W^{n}}:=\sigma\left\{w^{n}(k, s), \mu_{s}^{n} ; k \in \mathbb{R}, s \leqslant t\right\}$ and $\left(\mu^{n}, w^{n}\right)$ is independent of $\left(\beta^{n}, \xi^{n}\right)$ under $\mathbb{P}^{n}$.

For $n=1,2$, define the process $\left(h_{t}^{n}\right)_{t \in[0, T]}$ by $h_{t}^{n}(k):=\frac{1}{f(k)}\langle k\rangle^{-\eta}\left(\lambda\left(k, \mu_{t}^{n}\right)-\widetilde{\lambda}\left(k, \mu_{t}^{n}\right)\right)$ for every $k \in \mathbb{R}$ and for every $t \in[0, T]$. It is $\left(\mathcal{G}_{t}^{\mu^{n}}\right)_{t \in[0, T]}$-adapted, $\int_{0}^{T} \int_{\mathbb{R}}\left|h_{t}^{n}(k)\right|^{2} \mathrm{~d} k \mathrm{~d} t$ is bounded and $\left(h_{t}^{n}\right)_{t \in[0, T]}$ satisfies for every $x \in \mathbb{R}$ and for every $t \in[0, T]$

$$
(b-\widetilde{b})\left(x, \mu_{t}^{n}\right)=\int_{\mathbb{R}} f(k)\left(\cos (k x) h_{t}^{\Re, i}(k)+\sin (k x) h_{t}^{\Im, i}(k)\right) \mathrm{d} k .
$$

Let us define $\mathbb{Q}^{n}$ as the absolutely continuous probability measure with respect to $\mathbb{P}^{n}$ with density

$$
\frac{\mathrm{d} \mathbb{Q}^{n}}{\mathrm{~d} \mathbb{P}^{n}}=\exp \left(-\int_{0}^{T} \int_{\mathbb{R}} \Re\left(\overline{h_{t}^{n}(k)} \mathrm{d} w^{n}(k, t)\right)-\frac{1}{2} \int_{0}^{T} \int_{\mathbb{R}}\left|h_{t}^{n}(k)\right|^{2} \mathrm{~d} k \mathrm{~d} t\right) .
$$

Let us denote $\mathrm{d} \widetilde{w}^{n}(k, t)=\mathrm{d} w^{n}(k, t)+h_{t}^{n}(k) \mathrm{d} k \mathrm{~d} t$. It follows from Girsanov's Theorem that $\mathcal{L}^{\mathbb{Q}^{n}}\left(\widetilde{w}^{n}, \beta^{n}, \xi^{n}\right)=\mathcal{L}^{\mathbb{P}^{n}}\left(w^{n}, \beta^{n}, \xi^{n}\right)$ and that, for any $t \in[0, T]$, the $\sigma$-field $\sigma\left\{\widetilde{w}^{n}\left(k, t^{\prime}\right)-\widetilde{w}^{n}(k, t)\right.$, $\left.\beta_{t^{\prime}}^{n}-\beta_{t}^{n}, k \in \mathbb{R}, t^{\prime} \in[t, T]\right\}$ is independent of $\mathcal{G}_{t}^{n}$ under $\mathbb{Q}^{n}$. Moreover, $\left(z_{t}^{n}\right)_{t \in[0, T]}$ satisfies

$$
z_{t}^{n}=\xi^{n}+\int_{0}^{t} \int_{\mathbb{R}} f(k) \Re\left(e^{-i k z_{s}^{n}} \mathrm{~d} \widetilde{w}^{n}(k, s)\right)+\beta_{t}^{n}+\int_{0}^{t} \widetilde{b}\left(z_{s}^{n}, \mu_{s}^{n}\right) \mathrm{d} s .
$$

Let us remark that $\left(\exp \left(-\int_{0}^{t} \int_{\mathbb{R}} \Re\left(\overline{h_{s}^{n}(k)} \mathrm{d} w^{n}(k, s)\right)-\frac{1}{2} \int_{0}^{t} \int_{\mathbb{R}}\left|h_{s}^{n}(k)\right|^{2} \mathrm{~d} k \mathrm{~d} s\right)\right)_{t \in[0, T]}$ and $\widetilde{w}_{\cdot \wedge t}^{n}=$

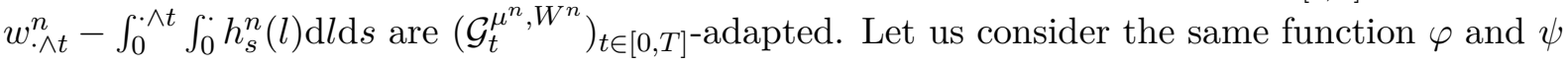


as in equality (63). We obtain by a similar computation:

$$
\begin{aligned}
\mathbb{E}^{\mathbb{Q}^{n}}\left[\psi\left(z_{t}^{n}\right) \varphi\left(\mu_{\cdot \wedge t}^{n}, \widetilde{w}_{\cdot \wedge t}^{n}\right)\right] \\
=\mathbb{E}^{\mathbb{P}^{n}}\left[\psi\left(z_{t}^{n}\right) \varphi\left(\mu_{\cdot \wedge t}^{n}, \widetilde{w}_{\cdot \wedge t}^{n}\right) \exp \left(-\int_{0}^{t} \int_{\mathbb{R}} \Re\left(\overline{h_{s}^{n}(k)} \mathrm{d} w^{n}(k, s)\right)-\frac{1}{2} \int_{0}^{t} \int_{\mathbb{R}}\left|h_{s}^{n}(k)\right|^{2} \mathrm{~d} k \mathrm{~d} s\right)\right] \\
=\mathbb{E}^{\mathbb{P}^{n}}\left[\mathbb{E}^{\mathbb{P}^{n}}\left[\psi\left(z_{t}^{n}\right) \mid \mathcal{G}_{t}^{\mu^{n}, W^{n}}\right] \varphi\left(\mu_{\cdot \wedge t}^{n}, \widetilde{w}_{\cdot \wedge t}^{n}\right)\right. \\
\left.\quad \cdot \exp \left(-\int_{0}^{t} \int_{\mathbb{R}} \Re\left(\overline{h_{s}^{n}(k)} \mathrm{d} w^{n}(k, s)\right)-\frac{1}{2} \int_{0}^{t} \int_{\mathbb{R}}\left|h_{s}^{n}(k)\right|^{2} \mathrm{~d} k \mathrm{~d} s\right)\right] \\
=\mathbb{E}^{\mathbb{P}^{n}}\left[\int_{\mathbb{R}} \psi(x) \mathrm{d} \mu_{t}^{n}(x) \varphi\left(\mu_{\cdot \wedge t}^{n}, \widetilde{w}_{\cdot \wedge t}^{n}\right) \exp \left(-\int_{0}^{t} \int_{\mathbb{R}} \Re\left(\overline{h_{s}^{n}(k)} \mathrm{d} w^{n}(k, s)\right)-\frac{1}{2} \int_{0}^{t} \int_{\mathbb{R}}\left|h_{s}^{n}(k)\right|^{2} \mathrm{~d} k \mathrm{~d} s\right)\right] \\
=\mathbb{E}^{\mathbb{Q}^{n}}\left[\int_{\mathbb{R}} \psi(x) \mathrm{d} \mu_{t}^{n}(x) \varphi\left(\mu_{\cdot \wedge t}^{n}, \widetilde{w}_{\cdot \wedge t}^{n}\right)\right],
\end{aligned}
$$

and thus for every $t \in[0, T], \mathbb{Q}^{n}$-almost surely, $\mu_{t}^{n}=\mathcal{L}^{\mathbb{Q}^{n}}\left(z_{t}^{n} \mid \mathcal{G}_{t}^{\mu^{n}, \widetilde{W}^{n}}\right)$.

Moreover $\left(\mu^{n}, \widetilde{w}^{n}\right)$ and $\frac{\mathrm{d} \mathbb{Q}^{n}}{\mathrm{~d} \mathbb{P}^{n}}$ are $\mathcal{G}^{\mu^{n}, W^{n}}$-measurable and under $\mathbb{P}^{n},\left(\mu^{n}, w^{n}\right)$ is independent of $\left(\beta^{n}, \xi^{n}\right)$. Thus for any bounded and measurable functions $g: \mathcal{C}([0, T], \mathbb{R}) \times \mathbb{R} \rightarrow \mathbb{R}$ and $f: \mathcal{C}\left([0, T], \mathcal{P}_{2}(\mathbb{R})\right) \times \mathcal{C}\left(\mathbb{R} \times[0, T], \mathbb{R}^{2}\right) \rightarrow \mathbb{R}$, we have

$$
\begin{aligned}
\mathbb{E}^{\mathbb{Q}^{n}}\left[f\left(\mu^{n}, \widetilde{w}^{n}\right) g\left(\beta^{n}, \xi^{n}\right)\right] & =\mathbb{E}^{\mathbb{P}^{n}}\left[f\left(\mu^{n}, \widetilde{w}^{n}\right) \frac{\mathrm{d} \mathbb{Q}^{n}}{\mathrm{~d} \mathbb{P}^{n}} g\left(\beta^{n}, \xi^{n}\right)\right] \\
& =\mathbb{E}^{\mathbb{P}^{n}}\left[f\left(\mu^{n}, \widetilde{w}^{n}\right) \frac{\mathrm{d} \mathbb{Q}^{n}}{\mathrm{~d} \mathbb{P}^{n}}\right] \cdot \mathbb{E}^{\mathbb{P}^{n}}\left[g\left(\beta^{n}, \xi^{n}\right)\right] \\
& =\mathbb{E}^{\mathbb{Q}^{n}}\left[f\left(\mu^{n}, \widetilde{w}^{n}\right)\right] \cdot \mathbb{E}^{\mathbb{Q}^{n}}\left[g\left(\beta^{n}, \xi^{n}\right)\right] .
\end{aligned}
$$

Thus under $\mathbb{Q}^{n},\left(\mu^{n}, \widetilde{w}^{n}\right)$ is independent of $\left(\beta^{n}, \xi^{n}\right)$. By the same argument and by the compatibility property under $\mathbb{P}^{n}$, we get that, under $\mathbb{Q}^{n}$, for any $t \in[0, T],\left(\xi^{n}, \widetilde{w}^{n}, \mu^{n}\right)$ and $\beta^{n}$ are conditionally independent given $\mathcal{G}_{t}^{n}$, which proves compatibility under $\mathbb{Q}^{n}$.

Thus we deduce that for $n=1,2,\left(\Omega^{n}, \mathcal{G}^{n},\left(\mathcal{G}_{t}^{n}\right)_{t \in[0, T]}, \mathbb{Q}^{n}, z^{n}, \widetilde{w}^{n}, \beta^{n}, \xi^{n}\right)$ are weak solutions to the SDE (38) with drift $\widetilde{b}$. By Proposition 28, it follows that $\mathcal{L}^{\mathbb{Q}^{1}}\left(z^{1}, \widetilde{w}^{1}\right)=\mathcal{L}^{\mathbb{Q}^{2}}\left(z^{2}, \widetilde{w}^{2}\right)$ and that for every $t \in[0, T], \mu_{t}^{n}=\mathcal{L}^{\mathbb{Q}^{n}}\left(z_{t}^{n} \mid \mathcal{G}_{t}^{\widetilde{W}^{n}}\right)$. Then, we apply the same computation as (29): for each bounded and measurable $\phi: \mathcal{C}([0, T], \mathbb{R}) \rightarrow \mathbb{R}$,

$$
\mathbb{E}^{\mathbb{P}^{n}}\left[\phi\left(z^{n}\right)\right]=\mathbb{E}^{\mathbb{Q}^{n}}\left[\phi\left(z^{n}\right) \exp \left(\int_{0}^{T} \int_{\mathbb{R}} \Re\left(\overline{h_{t}^{n}(k)} \mathrm{d} \widetilde{w}^{n}(k, t)\right)-\frac{1}{2} \int_{0}^{T} \int_{\mathbb{R}}\left|h_{t}^{n}(k)\right|^{2} \mathrm{~d} k \mathrm{~d} t\right)\right] .
$$

Recall that $h_{t}^{n}(k)=\frac{1}{f(k)}\langle k\rangle^{-\eta}\left(\lambda\left(k, \mu_{t}^{n}\right)-\tilde{\lambda}\left(k, \mu_{t}^{n}\right)\right)$ and that $\mu_{t}^{n}=\mathcal{L}^{\mathbb{Q}^{n}}\left(z_{t}^{n} \mid \mathcal{G}_{t}^{\widetilde{W}^{n}}\right)$. Hence the process $\left(h_{t}^{n}\right)_{t \in[0, T]}$ is $\left(\mathcal{G}_{t}^{W^{n}}\right)_{t \in[0, T]}$-progressively measurable. It follows that there is a measurable map $\psi: \mathcal{C}\left(\mathbb{R} \times[0, T], \mathbb{R}^{2}\right) \rightarrow \mathbb{R}$, independent of $n$, such that $\mathbb{E}^{\mathbb{Q}^{n}}\left[\left|\psi\left(\widetilde{w}^{n}\right)\right|\right]<+\infty$ and

$$
\mathbb{E}^{\mathbb{P}^{1}}\left[\phi\left(z^{1}\right)\right]=\mathbb{E}^{\mathbb{Q}^{1}}\left[\phi\left(z^{1}\right) \psi\left(\widetilde{w}^{1}\right)\right]=\mathbb{E}^{\mathbb{Q}^{2}}\left[\phi\left(z^{2}\right) \psi\left(\widetilde{w}^{2}\right)\right]=\mathbb{E}^{\mathbb{P}^{2}}\left[\phi\left(z^{2}\right)\right] .
$$

We conclude that $\mathcal{L}^{\mathbb{P}^{1}}\left(z^{1}\right)=\mathcal{L}^{\mathbb{P}^{2}}\left(z^{2}\right)$. This completes the proof of Theorem 32 .

Remark 35 . As the last computation right above highlights it, we have in fact that $\mathcal{L}^{\mathbb{P}^{1}}\left(z^{1}, \widetilde{w}^{1}\right)=$ $\mathcal{L}^{\mathbb{P}^{2}}\left(z^{2}, \widetilde{w}^{2}\right)$ and then $\mathcal{L}^{\mathbb{P}^{1}}\left(z^{1}, \mu^{1}\right)=\mathcal{L}^{\mathbb{P}^{2}}\left(z^{2}, \mu^{2}\right)$. 


\section{References}

[CD18] R. Carmona and F. Delarue. Probabilistic theory of mean field games with applications. II, volume 84 of Probability Theory and Stochastic Modelling. Springer, Cham, 2018. Mean field games with common noise and master equations.

[CdRF] P.-E. Chaudru de Raynal and N. Frikha. Well-posedness for some non-linear diffusion processes and related PDE on the Wasserstein space. arXiv preprint arXiv:1811.06904, 2018.

[CF16] M. Coghi and F. Flandoli. Propagation of chaos for interacting particles subject to environmental noise. Ann. Appl. Probab., 26(3):1407-1442, 2016.

[Del19] F. Delarue. Restoring uniqueness to mean-field games by randomizing the equilibria. Stochastics and Partial Differential Equations: Analysis and Computations, Mar 2019 .

[DPF10] G. Da Prato and F. Flandoli. Pathwise uniqueness for a class of SDE in Hilbert spaces and applications. J. Funct. Anal., 259(1):243-267, 2010.

[DPFPR13] G. Da Prato, F. Flandoli, E. Priola, and M. Röckner. Strong uniqueness for stochastic evolution equations in Hilbert spaces perturbed by a bounded measurable drift. Ann. Probab., 41(5):3306-3344, 2013.

[DPFPR15] G. Da Prato, F. Flandoli, E. Priola, and M. Röckner. Strong uniqueness for stochastic evolution equations with unbounded measurable drift term. J. Theoret. Probab., 28(4):1571-1600, 2015.

[DV95] D. Dawson and J. Vaillancourt. Stochastic McKean-Vlasov equations. NoDEA Nonlinear Differential Equations Appl., 2(2):199-229, 1995.

[FFPV17] E. Fedrizzi, F. Flandoli, E. Priola, and J. Vovelle. Regularity of stochastic kinetic equations. Electron. J. Probab., 22:Paper No. 48, 42, 2017.

[FGP10] F. Flandoli, M. Gubinelli, and E. Priola. Well-posedness of the transport equation by stochastic perturbation. Invent. Math., 180(1):1-53, 2010.

[Fla11] F. Flandoli. Random perturbation of PDEs and fluid dynamic models, volume 2015 of Lecture Notes in Mathematics. Springer, Heidelberg, 2011. Lectures from the 40th Probability Summer School held in Saint-Flour, 2010, École d'Été de Probabilités de Saint-Flour. [Saint-Flour Probability Summer School].

[Fun84] T. Funaki. A certain class of diffusion processes associated with nonlinear parabolic equations. Z. Wahrsch. Verw. Gebiete, 67(3):331-348, 1984.

[G̈88] J. Gärtner. On the McKean-Vlasov limit for interacting diffusions. Math. Nachr., 137:197-248, 1988.

[GLL11] O. Guéant, J.-M. Lasry, and P.-L. Lions. Mean field games and applications. In Paris-Princeton Lectures on Mathematical Finance 2010, volume 2003 of Lecture Notes in Math., pages 205-266. Springer, Berlin, 2011.

[GM11] L. Gawarecki and V. Mandrekar. Stochastic differential equations in infinite dimensions with applications to stochastic partial differential equations. Probability and its Applications (New York). Springer, Heidelberg, 2011. 
[Jou97] B. Jourdain. Diffusions with a nonlinear irregular drift coefficient and probabilistic interpretation of generalized Burgers' equations. ESAIM Probab. Statist., 1:339$355,1995 / 97$.

[Kon11] V. V. Konarovskii. On an infinite system of diffusing particles with coalescing. Theory Probab. Appl., 55(1):134-144, 2011.

[Kon17a] V. Konarovskyi. On asymptotic behavior of the modified Arratia flow. Electron. J. Probab., 22:Paper No. 19, 31, 2017.

[Kon17b] V. Konarovskyi. A system of coalescing heavy diffusion particles on the real line. Ann. Probab., 45(5):3293-3335, 2017.

[KR05] N. V. Krylov and M. Röckner. Strong solutions of stochastic equations with singular time dependent drift. Probab. Theory Related Fields, 131(2):154-196, 2005.

[KS91] I. Karatzas and S. E. Shreve. Brownian motion and stochastic calculus, volume 113 of Graduate Texts in Mathematics. Springer-Verlag, New York, second edition, 1991.

[Kur07] T. G. Kurtz. The Yamada-Watanabe-Engelbert theorem for general stochastic equations and inequalities. Electron. J. Probab., 12:951-965, 2007.

[Kur14] T. G. Kurtz. Weak and strong solutions of general stochastic models. Electron. Commun. Probab., 19:no. 58, 16, 2014.

[KvR18] V. Konarovskyi and M.-K. von Renesse. Modified Massive Arratia Flow and Wasserstein Diffusion. Communications on Pure and Applied Mathematics, 2018. doi:10.1002/cpa.21758.

[KX99] T. G. Kurtz and J. Xiong. Particle representations for a class of nonlinear SPDEs. Stochastic Process. Appl., 83(1):103-126, 1999.

[KX04] T. G. Kurtz and J. Xiong. A stochastic evolution equation arising from the fluctuations of a class of interacting particle systems. Commun. Math. Sci., 2(3):325-358, 2004 .

[Lac18] D. Lacker. On a strong form of propagation of chaos for McKean-Vlasov equations. Electron. Commun. Probab., 23:Paper No. 45, 11, 2018.

[Mar18] V. Marx. A new approach for the construction of a Wasserstein diffusion. Electron. J. Probab., 23:Paper No. 124, 54, 2018.

[Mar19] V. Marx. Diffusive processes on the Wasserstein space: Coalescing models, Regularization properties and McKean-Vlasov equations. Phd thesis, Université Côte d'Azur, October 2019. HAL-ID: tel-02342939.

[MV] Y. S. Mishura and A. Y. Veretennikov. Existence and uniqueness theorems for solutions of McKean-Vlasov stochastic equations. arXiv preprint arXiv:1603.02212, 2016.

[Oel84] K. Oelschläger. A martingale approach to the law of large numbers for weakly interacting stochastic processes. Ann. Probab., 12(2):458-479, 1984.

[Par67] K. R. Parthasarathy. Probability measures on metric spaces. Probability and Mathematical Statistics, No. 3. Academic Press, Inc., New York-London, 1967. 
[RY99] D. Revuz and M. Yor. Continuous martingales and Brownian motion, volume 293 of Grundlehren der Mathematischen Wissenschaften [Fundamental Principles of Mathematical Sciences]. Springer-Verlag, Berlin, third edition, 1999.

[RZ] M. Röckner and X. Zhang. Well-posedness of distribution dependent SDEs with singular drifts. arXiv preprint arXiv:1809.02216, 2018.

[SV69] D. W. Stroock and S. R. S. Varadhan. Diffusion processes with continuous coefficients i\&ii. Comm. Pure Appl. Math., 22:345-400 \& 479-530, 1969.

[SV79] D. W. Stroock and S. R. S. Varadhan. Multidimensional diffusion processes, volume 233 of Grundlehren der Mathematischen Wissenschaften [Fundamental Principles of Mathematical Sciences]. Springer-Verlag, Berlin-New York, 1979.

[Szn91] A.-S. Sznitman. Topics in propagation of chaos. In École d'Été de Probabilités de Saint-Flour XIX-1989, volume 1464 of Lecture Notes in Math., pages 165-251. Springer, Berlin, 1991.

[Vai88] J. Vaillancourt. On the existence of random McKean-Vlasov limits for triangular arrays of exchangeable diffusions. Stochastic Anal. Appl., 6(4):431-446, 1988.

[Ver80] A. J. Veretennikov. Strong solutions and explicit formulas for solutions of stochastic integral equations. Mat. Sb. (N.S.), 111(153)(3):434-452, 480, 1980.

[vRS09] M.-K. von Renesse and K.-T. Sturm. Entropic measure and Wasserstein diffusion. Ann. Probab., 37(3):1114-1191, 2009.

[Wal86] J. B. Walsh. An introduction to stochastic partial differential equations. In École d'été de probabilités de Saint-Flour, XIV-1984, volume 1180 of Lecture Notes in Math., pages 265-439. Springer, Berlin, 1986.

[Zvo74] A. K. Zvonkin. A transformation of the phase space of a diffusion process that will remove the drift. Mat. Sb. (N.S.), 93(135):129-149, 152, 1974. 